\title{
From Heterocycles to Carbacycles: Synthesis of Carbocyclic Nucleoside Analogues from Enals and Hydroxylamines
}

\author{
Pulakesh Das, David W. Almond, Lauren N. Tumbelty, Brooke E. Austin and Gustavo Moura- \\ Letts* \\ Department of Chemistry and Biochemistry, College of Science and Mathematics \\ Rowan University \\ 201 Mullica Hill Rd, Glassboro, NJ, 08028
}

\section{Supporting Information}
A. Materials and Methods
S1
B. Synthesis of BIX from Figure 1 and Figure 2
S5
C. Synthesis of carbacycles from Figure 1 and Figure $2 \quad \mathrm{~S} 13$
D. X-ray crystallography data
$\mathrm{S} 14$
E. ${ }^{1} \mathrm{H}-\mathrm{NMR}$ and ${ }^{13} \mathrm{C}$-MR spectra
S26 


\section{A. MATERIALS AND METHODS}

Reagents were obtained from Aldrich Chemical (www.sigma-aldrich.com), Acros Organics (www.us.vwr.com) or Alfa Aesar (www.us.vwr.com) and used without further purification. Solvents were obtained from EMD Miliphore DrySol (www.us.vwr.com) and degassed with $\mathrm{N}_{2}$. Solution phase reactions were performed in glass vials or round bottom flasks with inert atmosphere and magnetic stirring. Cold baths were generated as follows: $0{ }^{\circ} \mathrm{C}$, wet ice/water; $10{ }^{\circ} \mathrm{C}$, ice/acetone; $-20{ }^{\circ} \mathrm{C}$, dry ice/isopropanol monitored with a thermometer; $-44{ }^{\circ} \mathrm{C}$, dry ice $/ \mathrm{CH}_{3} \mathrm{CN}$; $-63{ }^{\circ} \mathrm{C}$, dry ice/chloroform; $-78{ }^{\circ} \mathrm{C}$, dry ice/acetone; $-100{ }^{\circ} \mathrm{C}$, dry ice/Et $2 \mathrm{O}$. Heated reactions were performed using IKA heating blocks.

TLC was performed on $0.25 \mathrm{~mm}$ E. Merck silica gel 60 F254 plates and visualized under UV light and/or the following stain solutions: cerium ammonium molybdate (CAM), phosphomolybdic acid (PMA), iodine $\left(\mathrm{I}_{2}\right)$, or $p$-anisaldehyde. Silica flash chromatography was performed on E. Merck 230-400 mesh silica gel 60. Automated chromatography was performed on a ISOLERA Prime instrument with $10 \mathrm{~g}$. SNAP silica gel normal phase cartridges using a flow rate of $12.0 \mathrm{~mL} / \mathrm{min}$ and a gradient of $0-100 \%$ EtOAc in heptanes over 20 min with UV detection at $254 \mathrm{~nm}$. NMR spectra were recorded on a Bruker Avance Neo $400 \mathrm{MHz}$ Spectrometer at $24{ }^{\circ} \mathrm{C}$ in $\mathrm{CDCl}_{3}$ unless otherwise indicated. Chemical shifts are expressed in ppm relative to TMS $\left({ }^{1} \mathrm{H}, 0 \mathrm{ppm}\right)$ or solvent signals: $\mathrm{CDCl}_{3}\left({ }^{1} \mathrm{H}, 7.23 \mathrm{ppm} ;{ }^{13} \mathrm{C}, 77.0 \mathrm{ppm}\right)$; coupling constants are expressed in $\mathrm{Hz}$. Low and high resolution mass spectroscopy was performed on a Agilent 6230 Accurate-Mass Time-ofFlight 1290 Infinity UHPLC/MS. X-Ray spectroscopy was performed on a Bruker Single Crystal $\mathrm{X}$-ray Diffractometer at Princeton University.

\section{General method for the synthesis of carbacycles:}

In a $20 \mathrm{~mL}$ vial, conjugated aldehyde $(0.1 \mathrm{mmol}, 1$ equiv. $)$ and alkylhydroxylamine $(0.11 \mathrm{mmol}$, 1.1 equiv.) were dissolved in ACN $(2 \mathrm{~mL})$. The resulting mixture was allowed to react for $2 \mathrm{~h}$ at room temperature. Methacrolein $(0.5 \mathrm{mmol}, 5$ equiv. $)$ was then added and the resulting mixture was heated to $80^{\circ} \mathrm{C}$ for $6 \mathrm{~h}$ or until disappearance of starting material by TLC. The solvent and excess methacrolein was then removed under vacuum and the resulting crude was reconstituted in $\mathrm{ACN}(4 \mathrm{~mL})$ and alkylhydroxylamine $(0.15 \mathrm{mmol}, 1.5$ equiv. $)$ was added and the resulting mixture was allowed to stir until disappearance of IX by TLC. The resulting mixture is then reacted with 1,2-ethanediol (0.05 mmol, 0.5 equiv.) and pTSA (0.01 mmol, $10 \mathrm{~mol} \%$ ) to scavenge any potential unreacted 3,4-endo-IX. The crude was then dried under vacuum and purified by silica gel flash chromatography to provide the resulting BIX as single diastereomer. Purified BIX was then dissolved in $\mathrm{H}_{2} \mathrm{O} / \mathrm{CH}_{2} \mathrm{Cl}_{2} / \mathrm{AcOH}(1: 1: 1,2 \mathrm{~mL})$ and to the resulting mixture $\mathrm{Zn}$ (4 mmol, 40 equiv. $)^{\mathrm{a}}$ was added. The reaction was stirred at room temperature for $12 \mathrm{~h}$ or until complete consumption of BIX by TLC. The resulting crude was then filtered through a pad of a 1:1 mixture of celite and silica gel and then dried and washed with pentanes (4 washes). The resulting CB was then characterized without further purification.

Large scale synthesis of 2a: In a $200 \mathrm{~mL}$ vial, cinnamaldehyde (650 mg, $5 \mathrm{mmol}, 1$ equiv.) and benzylhydroxylamine (677 mg, $5.5 \mathrm{mmol}, 1.1$ equiv.) were dissolved in ACN (100 mL). The resulting mixture was allowed to react for $2 \mathrm{~h}$ at room temperature. Methacrolein $(25 \mathrm{mmol}, 5$ equiv.) was then added and the resulting mixture was heated to $80^{\circ} \mathrm{C}$ for $6 \mathrm{~h}$ or until disappearance of starting material by TLC. The solvent and excess methacrolein was then removed under vacuum and the resulting crude was reconstituted in ACN $(4 \mathrm{~mL})$ and benzylhydroxylamine $(975 \mathrm{mg}, 7.5$ mmol, 1.5 equiv.) was added and the resulting mixture was allowed to stir until disappearance of IX by TLC. The crude was then dried under vacuum and purified by silica gel flash chromatography to provide the resulting BIX as single diastereomer (1689 mg, 83\%). Purified BIX was then dissolved in $\mathrm{H}_{2} \mathrm{O} / \mathrm{CH}_{2} \mathrm{Cl}_{2} / \mathrm{AcOH}(1: 1: 1,100 \mathrm{~mL})$ and to the resulting mixture $\mathrm{Zn}(13 \mathrm{~g}$, $200 \mathrm{mmol}, 40$ equiv.) was added. The reaction was stirred at room temperature for $12 \mathrm{~h}$ or until complete consumption of BIX by TLC. The resulting crude was then filtered through a pad of a 
1:1 mixture of celite and silica gel and then dried and washed with pentanes (4 washes). The resulting CB $2 \mathrm{a}(1637 \mathrm{mg}, 96 \%)$ was then characterized without further purification.

Mechanism experiments: The selectivity of the intramolecular dipolar cycloaddition was corroborated by monitoring the reaction by ${ }^{1} \mathrm{H}-\mathrm{NMR}$ (Figure S1). The high selectivity for the endoexo-BIX is clearly highlighted in the monitoring experiment below. As nitrone is formed (shift of alkene resonances) slowly gets consumed to form the observed BIX. Complete conversion for this reaction was observed around 50h (NMR tube was sonicated at $\mathrm{rt}$ ).

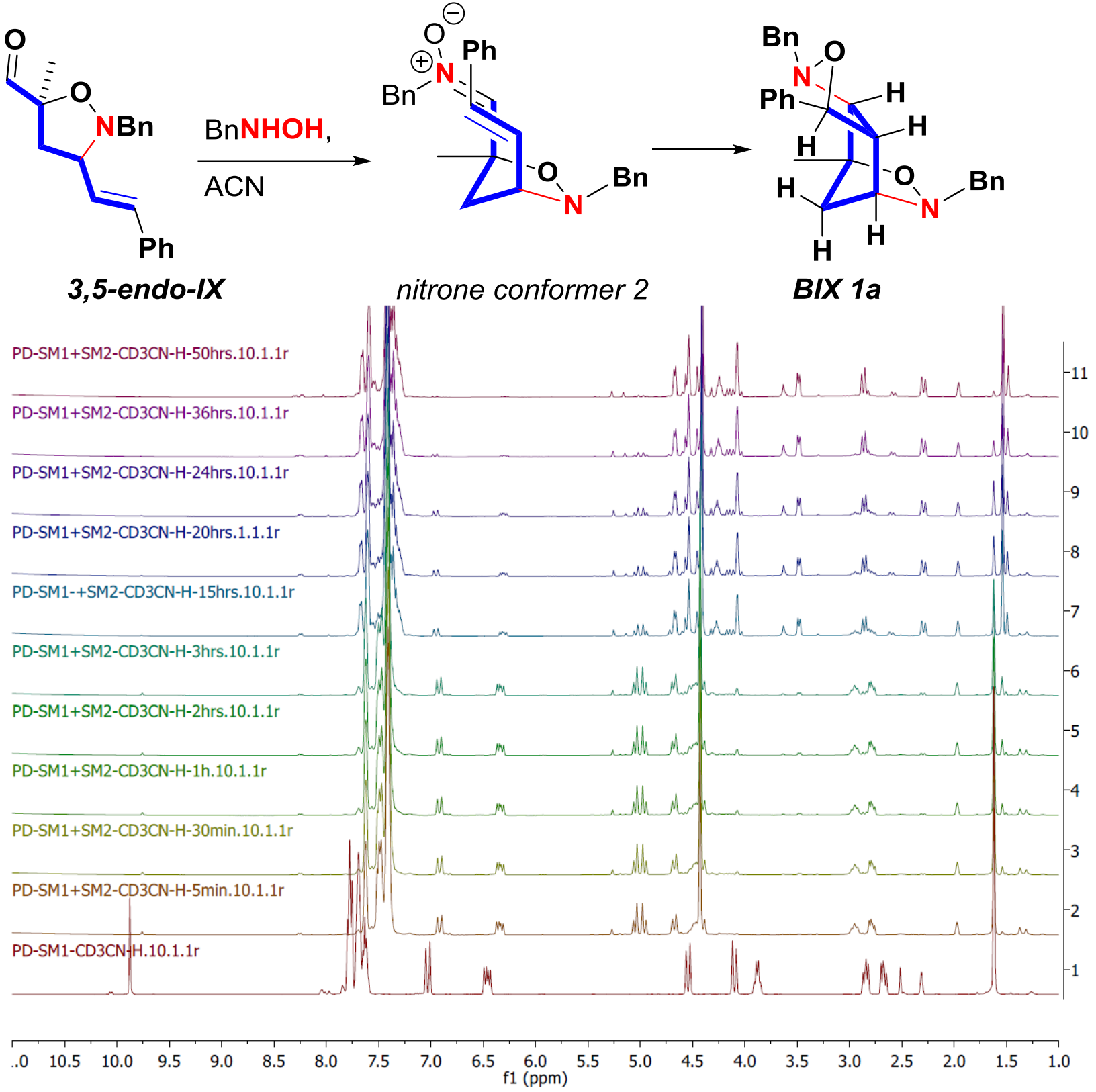

Figure S1. Reaction monitoring by ${ }^{1} \mathrm{H}-\mathrm{NMR}$.

${ }^{\text {a }}$ Despite the acidic reaction conditions, the carbacycles are isolated as free alkyl amines due to the neutralizing effect from the high excess of Zn. 
Substrates that failed to provide CB under the developed method: These substrates (as the conjugated aldehyde component) either provided low conversions or complex mixtures that required multiple purifications.<smiles>C=CC=O</smiles><smiles>C/C=C(\C=O)c1ccccc1</smiles><smiles>C/C=C/C=O</smiles><smiles>C=CC(=O)CC</smiles><smiles>CC(C)=CC=O</smiles><smiles>C/C(C=O)=C\P</smiles><smiles>CC=CC=O</smiles><smiles>C=CC(C)=O</smiles>

Scheme S1. Failed reaction substrates 


\section{B. SYNTHESIS OF BRIDGED-BISISOXAZOLIDINES FROM TABLE 1:}

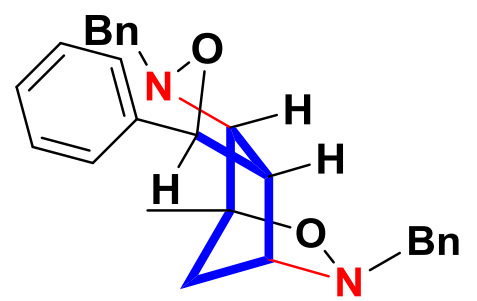

\section{1,5-dibenzyl-7-methyl-3-phenylhexahydro-1H-4,7-methanoisoxazolo[4,3-d][1,2]}

(1a): Cinnamaldehyde (13 $\mathrm{mg}, 0.1 \mathrm{mmol})$ was reacted using general method and the resulting BIX was purified by silica gel flash chromatography (10:1 heptanes/EtOAc), yielding 1 a (33 mg, $80 \%$ ) as a white solid. MP: $128-130^{\circ} \mathrm{C}$. TLC: $R_{f} 0.62$ (2:1 heptanes/EtOAc). IR (thin film): 3062 , 2928, 1605, 1496, 1452, 1203, 1159, 1079, $1058 \mathrm{~cm}^{-1} .{ }^{1} \mathbf{H}-\mathbf{N M R}\left(400 \mathrm{MHz}, \mathrm{CDCl}_{3}\right): \delta$ 7.36-7.24 $(\mathrm{m}, 15 \mathrm{H}), 4.56(\mathrm{~d}, J=7.2 \mathrm{~Hz}, 1 \mathrm{H}), 4.08-3.96(\mathrm{~m}, 3 \mathrm{H}), 3.72(\mathrm{~d}, J=12.5 \mathrm{~Hz}, 1 \mathrm{H}), 3.44(\mathrm{~s}, 1 \mathrm{H}), 3.22$ $(\mathrm{dd}, J=8.4,1.5 \mathrm{~Hz}, 1 \mathrm{H}), 3.13(\mathrm{t}, J=7.8 \mathrm{~Hz}, 1 \mathrm{H}), 2.48(\mathrm{~d}, J=11.3 \mathrm{~Hz}, 1 \mathrm{H}), 1.98(\mathrm{dd}, J=11.3$, $1.9 \mathrm{~Hz}, 1 \mathrm{H}), 1.42(\mathrm{~s}, 3 \mathrm{H}) .{ }^{13} \mathbf{C}-\mathbf{N M R}\left(100 \mathrm{MHz}, \mathrm{CDCl}_{3}\right): \delta 139.4,137.2,136.9,129.4,129.3$, $129.2,128.7,128.6,128.5,128.3,128.2,128.1,128.0,127.5,127.4,127.0,126.9,126.8,85.5$, 82.1, 75.5, 63.7, 63.1, 62.4, 61.1, 34.1, $14.8 \mathrm{ppm}$. ESI-MS $\mathrm{m} / z$ (rel int): (pos) $413.2\left([\mathrm{M}+\mathrm{H}]^{+}\right.$, 100); (neg) 411.2 ([M-H] $\left.]^{-}, 100\right)$. HRMS (ESI): Calculated for: $\mathrm{C}_{27} \mathrm{H}_{29} \mathrm{~N}_{2} \mathrm{O}_{2}{ }^{+}$: 413.2224, found: 413.2231. Absolute difference (ppm): 1.69.

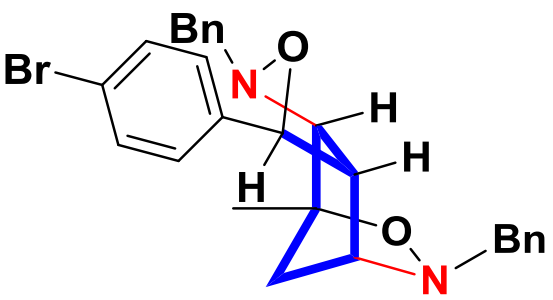

1,5-dibenzyl-3-(4-bromophenyl)-7-methylhexahydro-1H-4,7-methanoisoxazolo[4,3-

d][1,2] oxazine (1b): 4-Bromocinnamaldehyde $(21 \mathrm{mg}, 0.1 \mathrm{mmol})$ was reacted using general method and the resulting BIX was purified by silica gel flash chromatography (10:1 heptanes/EtOAc), yielding $\mathbf{1 b}(40 \mathrm{mg}, 82 \%)$ as a white solid. MP: $158-160^{\circ} \mathrm{C}$. TLC: $R_{f} 0.57(2: 1$ heptanes/EtOAc). IR (thin film): 3030, 2970, 2928, 1605, 1489, 1453, 1265, 1160, 1070, 1010 $\left.\mathrm{cm}^{-1} .{ }^{1} \mathrm{H}-\mathrm{NMR}\left(400 \mathrm{MHz}, \mathrm{CDCl}_{3}\right):\right): \delta 7.43(\mathrm{~d}, J=8.2 \mathrm{~Hz}, 2 \mathrm{H}), 7.39-7.22(\mathrm{~m}, 10 \mathrm{H}), 7.17(\mathrm{~d}, J=$ $8.2 \mathrm{~Hz}, 2 \mathrm{H}), 4.50(\mathrm{~d}, J=7.1 \mathrm{~Hz}, 1 \mathrm{H}), 4.15-3.92(\mathrm{~m}, 3 \mathrm{H}), 3.72(\mathrm{~d}, J=12.9 \mathrm{~Hz}, 1 \mathrm{H}), 3.42(\mathrm{~s}, 1 \mathrm{H})$, $3.20(\mathrm{dd}, J=8.4,1.6 \mathrm{~Hz}, 1 \mathrm{H}), 3.04(\mathrm{t}, J=7.8 \mathrm{~Hz}, 1 \mathrm{H}), 2.45(\mathrm{~d}, J=11.3 \mathrm{~Hz}, 1 \mathrm{H}), 1.98(\mathrm{dd}, J=$ $11.3,1.9 \mathrm{~Hz}, 1 \mathrm{H}), 1.55(\mathrm{~s}, 3 \mathrm{H}) .{ }^{13} \mathbf{C}-\mathbf{N M R}\left(100 \mathrm{MHz}, \mathrm{CDCl}_{3}\right): \delta 138.5,137.1,136.8,131.6,129.2$ (2C), 129.1, 128.5, 128.4 (2C), 128.3 (2C), 128.2 (2C), 127.5, 127.4 (2C), 121.9, 85.4, 81.4, 75.5, 63.5, 63.1, 62.4, 61.2, 34.1, 14.8 ppm. ESI-MS m/z (rel int): (pos) 491.1 ([M+H] $\left.]^{+}, 100\right)$; (neg) $489.1\left([\mathrm{M}-\mathrm{H}]^{-}, 100\right)$. HRMS (ESI): Calculated for: $\mathrm{C}_{27} \mathrm{H}_{28} \mathrm{BrN}_{2} \mathrm{O}_{2}{ }^{+}: 491.1329$, found: 491.1325. Absolute difference (ppm): 0.81. 


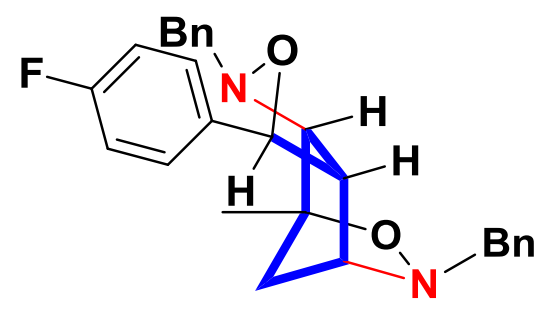

1,5-dibenzyl-3-(4-fluorophenyl)-7-methylhexahydro-1H-4,7-methanoisoxazolo[4,3-

d][1,2] oxazine (1c): 4-Fluorocinnamaldehyde (15 mg, $0.1 \mathrm{mmol})$ was reacted using general method and the resulting BIX was purified by silica gel flash chromatography (10:1 heptanes/EtOAc), yielding 1c (24 mg, 73\%) as a white solid. MP: $119-122^{\circ} \mathrm{C}$. TLC: $R_{f} 0.53(2: 1$ heptanes/EtOAc). IR (thin film): 3031, 2970, 1605, 1510, 1496, 1453, 1224, 1158, $1083 \mathrm{~cm}^{-1}$. ${ }^{1}$ H-NMR $\left(400 \mathrm{MHz}, \mathrm{CDCl}_{3}\right): \delta$ 7.44-7.18 (m, 12H), $6.99(\mathrm{dd}, J=9.5,7.9 \mathrm{~Hz}, 2 \mathrm{H}), 4.53(\mathrm{~d}, J=$ $7.2 \mathrm{~Hz}, 1 \mathrm{H}), 4.15-3.91(\mathrm{~m}, 3 \mathrm{H}), 3.72(\mathrm{~d}, J=12.9 \mathrm{~Hz}, 1 \mathrm{H}), 3.42(\mathrm{~s}, 1 \mathrm{H}), 3.21(\mathrm{dd}, J=8.5,1.7 \mathrm{~Hz}$, $1 \mathrm{H}), 3.06(\mathrm{t}, J=7.7 \mathrm{~Hz}, 1 \mathrm{H}), 2.48(\mathrm{~d}, J=11.2 \mathrm{~Hz}, 1 \mathrm{H}), 1.98(\mathrm{dd}, J=11.3,1.8 \mathrm{~Hz}, 1 \mathrm{H}), 1.55(\mathrm{~s}$, 3H). ${ }^{13} \mathrm{C}-\mathbf{N M R}\left(100 \mathrm{MHz}, \mathrm{CDCl}_{3}\right): \delta 162.1,137.2,136.8,135.1,135.0,129.2,129.1(2 \mathrm{C}), 128.6$, 128.5(2C), 128.4, 128.3, 128.2, 127.5, 127.4, 115.5 (2C), 85.4, 81.4, 75.5, 63.5, 63.1, 62.4, 61.3, 34.1, $14.8 \mathrm{ppm}$ ESI-MS m/z (rel int): (pos) 431.2 ([M+H] $\left.]^{+}, 100\right)$; (neg) 429.2 ([M-H] $\left.]^{-}, 100\right)$. HRMS (ESI): $[\mathrm{M}+\mathrm{H}]^{+}$Calculated for: $\mathrm{C}_{27} \mathrm{H}_{28} \mathrm{FN}_{2} \mathrm{O}_{2}{ }^{+}$: 431.2129, found: 431.2133. Absolute difference (ppm): 0.93 .

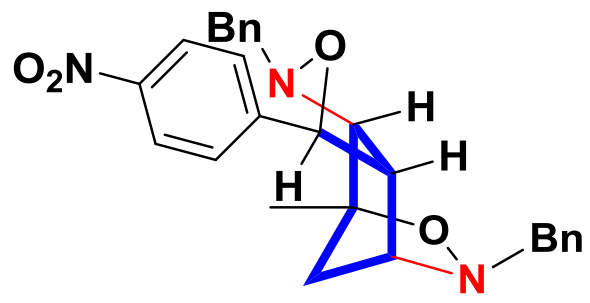

\section{1,5-dibenzyl-7-methyl-3-(4-nitrophenyl)hexahydro-1H-4,7-methanoisoxazolo[4,3-}

d][1,2]oxazine (1d): 4-Nitrocinnamaldehyde (18 mg, $0.1 \mathrm{mmol})$ was reacted using general method and the resulting BIX was purified by silica gel flash chromatography (10:1 heptanes/EtOAc), yielding 1d (36 mg, 79\%) as a light yellow solid. MP: $126-128^{\circ} \mathrm{C}$ TLC: $R_{f}$ 0.51 (2:1 heptanes/EtOAc). IR (thin film): 3031, 2972, 1605, 1519, 1347, 1204, 1150, $1064 \mathrm{~cm}^{-1} .{ }^{1} \mathbf{H}-\mathbf{N M R}$ $\left(400 \mathrm{MHz}, \mathrm{CDCl}_{3}\right): \delta 8.16(\mathrm{~d}, J=8.8 \mathrm{~Hz}, 2 \mathrm{H}), 7.57-7.16(\mathrm{~m}, 12 \mathrm{H}), 4.62(\mathrm{~d}, J=7.2 \mathrm{~Hz}, 1 \mathrm{H}), 4.08-$ $3.96(\mathrm{~m}, 3 \mathrm{H}), 3.74(\mathrm{~d}, J=12.9 \mathrm{~Hz}, 1 \mathrm{H}), 3.48(\mathrm{~s}, 1 \mathrm{H}), 3.23(\mathrm{dd}, J=8.3,1.6 \mathrm{~Hz}, 1 \mathrm{H}), 3.03(\mathrm{t}, J=$ $7.8 \mathrm{~Hz}, 1 \mathrm{H}), 2.47(\mathrm{~d}, J=11.3 \mathrm{~Hz}, 1 \mathrm{H}), 2.02(\mathrm{dd}, J=11.3,1.8 \mathrm{~Hz}, 1 \mathrm{H}), 1.45(\mathrm{~s}, 3 \mathrm{H}) .{ }^{13} \mathbf{C}-\mathbf{N M R}$ (100 MHz, $\left.\mathrm{CDCl}_{3}\right): \delta 147.6,146.9,136.9,136.6,129.2$ (2C), 129.1 (2C), 128.5 (2C), $128.3(2 \mathrm{C})$, 127.6 (2C), 127.2 (2C), 123.7 (2C), 85.4, 80.8, 75.5, 63.4, 63.1, 62.4, 61.5, 34.1, 14.8 ppm. ESI-MS $m / z$ (rel int): (pos) $458.2\left([\mathrm{M}+\mathrm{H}]^{+}, 100\right)$; (neg) 456.2 ([M-H $\left.]^{-}, 100\right)$. HRMS (ESI): $[\mathrm{M}+\mathrm{H}]^{+}$Calculated for: $\mathrm{C}_{27} \mathrm{H}_{28} \mathrm{~N}_{3} \mathrm{O}_{4}{ }^{+}: 458.2074$, found: 458.2082. Absolute difference (ppm): 1.75 . 


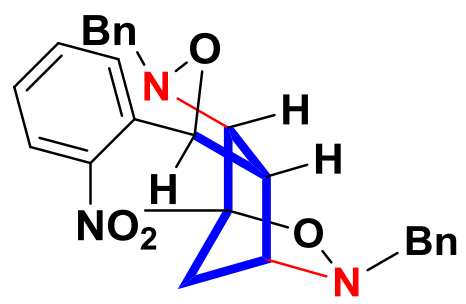

\section{1,5-dibenzyl-7-methyl-3-(2-nitrophenyl)hexahydro-1H-4,7-methanoisoxazolo[4,3-}

d] [1,2] oxazine (1e): 2-Nitrocinnamaldehyde (18 $\mathrm{mg}, 0.1 \mathrm{mmol})$ was reacted using general method and the resulting BIX was purified by silica gel flash chromatography (10:1 heptanes/EtOAc), yielding 1e (39 $\mathrm{mg}, 84 \%)$ as a light yellow solid. MP: $114-116^{\circ} \mathrm{C}$. TLC: $R_{f} 0.53(2: 1$ heptanes/EtOAc). IR (thin film): 3031, 2972, 1605, 1519, 1496, 1379, 1204, 1150, $1108 \mathrm{~cm}^{-1}$. ${ }^{1}$ H-NMR $\left(400 \mathrm{MHz}, \mathrm{CDCl}_{3}\right): \delta 7.81(\mathrm{~d}, J=8.3 \mathrm{~Hz}, 1 \mathrm{H}), 7.74(\mathrm{~d}, J=8.0 \mathrm{~Hz}, 1 \mathrm{H}), 7.59(\mathrm{t}, J=7.8$ $\mathrm{Hz}, 1 \mathrm{H}), 7.46-7.15(\mathrm{~m}, 11 \mathrm{H}), 5.10(\mathrm{~d}, J=7.5 \mathrm{~Hz}, 1 \mathrm{H}), 4.07(\mathrm{~s}, 2 \mathrm{H}), 4.00(\mathrm{~d}, J=12.9 \mathrm{~Hz}, 1 \mathrm{H}), 3.88$ $(\mathrm{s}, 1 \mathrm{H}), 3.76(\mathrm{~d}, J=13.0 \mathrm{~Hz}, 1 \mathrm{H}), 3.26(\mathrm{~d}, J=8.0 \mathrm{~Hz}, 1 \mathrm{H}), 3.01(\mathrm{t}, J=8.2 \mathrm{~Hz}, 1 \mathrm{H}), 2.51(\mathrm{~d}, J=$ $11.5 \mathrm{~Hz}, 1 \mathrm{H}), 2.04(\mathrm{~d}, J=11.5 \mathrm{~Hz}, 1 \mathrm{H}), 1.46(\mathrm{~s}, 3 \mathrm{H}) .{ }^{13} \mathbf{C}-\mathbf{N M R}\left(100 \mathrm{MHz}, \mathrm{CDCl}_{3}\right): \delta 148.8$, 137.1, 137.0, 135.2, 133.3, 129.3 (2C), 128.9 (2C), 128.8 (2C), 128.5 (2C), 128.4, 128.3, 127.5, 127.4, 124.2, 85.1 (2C), 75.8, 63.8, 62.8, 62.7, 62.5, 34.4, 14.9 ppm. ESI-MS $m / z$ (rel int): (pos) $458.2\left([\mathrm{M}+\mathrm{H}]^{+}, 100\right)$; (neg) $456.2\left([\mathrm{M}-\mathrm{H}]^{-}, 100\right)$. HRMS (ESI): $[\mathrm{M}+\mathrm{H}]^{+}$Calculated for: $\mathrm{C}_{27} \mathrm{H}_{28} \mathrm{~N}_{3} \mathrm{O}_{4}{ }^{+}: 458.2074$, found: 458.2067. Absolute difference (ppm): 1.53 .

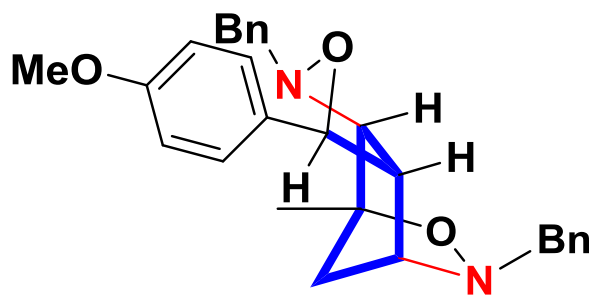

\section{1,5-dibenzyl-3-(4-methoxyphenyl)-7-methylhexahydro-1H-4,7-methanoisoxazolo[4,3-}

d][1,2] oxazine (1f): 4-Methoxycinnamaldehyde $(16 \mathrm{mg}, 0.1 \mathrm{mmol})$ was reacted using general method and the resulting BIX was purified by silica gel flash chromatography (10:1 heptanes/EtOAc), yielding $\mathbf{1 f}(38 \mathrm{mg}, 86 \%)$ as a white solid. MP: $165-169^{\circ} \mathrm{C}$. TLC: $R_{f} 0.50(2: 1$ heptanes/EtOAc). IR (thin film): 3030, 2929, 1612, 1514, 1496, 1453, 1304, 1203, 1160, 1028 $\mathrm{cm}^{-1} .{ }^{1} \mathbf{H}-\mathbf{N M R}\left(400 \mathrm{MHz}, \mathrm{CDCl}_{3}\right): \delta 7.49-7.12(\mathrm{~m}, 12 \mathrm{H}), 6.84(\mathrm{~d}, J=8.6 \mathrm{~Hz}, 2 \mathrm{H}), 4.52(\mathrm{~d}, J=$ $7.1 \mathrm{~Hz}, 1 \mathrm{H}), 4.10-3.92(\mathrm{~m}, 3 \mathrm{H}), 3.78(\mathrm{~s}, 3 \mathrm{H}), 3.71(\mathrm{~d}, J=12.9 \mathrm{~Hz}, 1 \mathrm{H}), 3.40(\mathrm{~s}, 1 \mathrm{H}), 3.21(\mathrm{dd}, J=$ 8.4, $1.5 \mathrm{~Hz}, 1 \mathrm{H}), 3.10(\mathrm{t}, J=7.8 \mathrm{~Hz}, 1 \mathrm{H}), 2.47(\mathrm{~d}, J=11.3 \mathrm{~Hz}, 1 \mathrm{H}), 2.02(\mathrm{dd}, J=11.3,1.8 \mathrm{~Hz}$, $1 \mathrm{H}), 1.41(\mathrm{~s}, 3 \mathrm{H}) .{ }^{13} \mathbf{C}-\mathbf{N M R}\left(100 \mathrm{MHz}, \mathrm{CDCl}_{3}\right): \delta 159.4,137.3,136.9,131.2,129.3,128.6,128.5$, 128.4, 128.3, 128.2, 128.1, 127.4 (2C), 127.3 (2C), 127.2, 113.8 (2C), 85.4, 81.9, 75.5, 63.5, 63.1, 62.4, 61.0, 55.3, 34.1, 14.9 ppm. ESI-MS $m / z$ (rel int): (pos) $443.2\left([\mathrm{M}+\mathrm{H}]^{+}, 100\right)$; (neg) 441.2 ([M-H] $\left.]^{-}, 100\right)$. HRMS (ESI): $[\mathrm{M}+\mathrm{H}]^{+}$Calculated for: $\mathrm{C}_{28} \mathrm{H}_{31} \mathrm{~N}_{2} \mathrm{O}_{3}{ }^{+}$: 443.2329, found: 443.2321 . Absolute difference (ppm): 1.81 . 


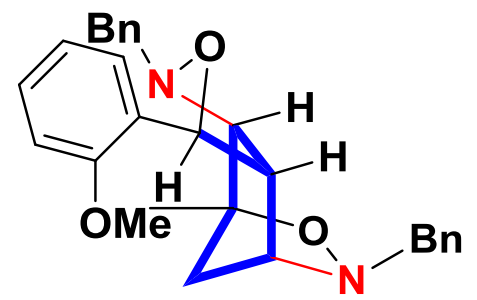

1,5-dibenzyl-3-(2-methoxyphenyl)-7-methylhexahydro-1H-4,7-methanoisoxazolo[4,3-

d][1,2] oxazine (1g): 2-Methoxycinnamaldehyde $(16 \mathrm{mg}, 0.1 \mathrm{mmol})$ was reacted using general method and the resulting BIX was purified by silica gel flash chromatography (10:1 heptanes/EtOAc), yielding $1 \mathrm{~g}(35 \mathrm{mg}, 79 \%)$ as a white solid. MP: $106-109{ }^{\circ} \mathrm{C}$. TLC: $R_{f} 0.58(2: 1$ heptanes/EtOAc). IR (thin film): 3032, 2930, 1603, 1494, 1454, 1265, 1245, 1160, $1028 \mathrm{~cm}^{-1}$. ${ }^{1}$ H-NMR $\left(400 \mathrm{MHz}, \mathrm{CDCl}_{3}\right): \delta 7.54-7.11(\mathrm{~m}, 12 \mathrm{H}), 6.90(\mathrm{td}, J=7.5,1.1 \mathrm{~Hz}, 1 \mathrm{H}), 6.75(\mathrm{dd}, J=$ $8.2,1.0 \mathrm{~Hz}, 1 \mathrm{H}), 4.81(\mathrm{~d}, J=7.0 \mathrm{~Hz}, 1 \mathrm{H}), 4.15(\mathrm{~d}, J=13.9 \mathrm{~Hz}, 1 \mathrm{H}), 4.10(\mathrm{~d}, J=12.5 \mathrm{~Hz}, 1 \mathrm{H})$, $4.01(\mathrm{~d}, J=13.9 \mathrm{~Hz}, 1 \mathrm{H}), 3.69(\mathrm{~d}, J=12.5 \mathrm{~Hz}, 1 \mathrm{H}), 3.66(\mathrm{~s}, 1 \mathrm{H}), 3.43(\mathrm{~s}, 3 \mathrm{H}), 3.14(\mathrm{~d}, J=8.4$, $1.6 \mathrm{~Hz}, 1 \mathrm{H}), 2.90(\mathrm{t}, J=7.7 \mathrm{~Hz}, 1 \mathrm{H}), 2.43(\mathrm{~d}, J=11.3 \mathrm{~Hz}, 1 \mathrm{H}), 2.02(\mathrm{dd}, J=11.3,1.8 \mathrm{~Hz}, 1 \mathrm{H})$, $1.45(\mathrm{~s}, 3 \mathrm{H}) .{ }^{13} \mathrm{C}-\mathbf{N M R}\left(100 \mathrm{MHz}, \mathrm{CDCl}_{3}\right): \delta 156.2,137.6,137.0,129.5,129.2,129.0(2 \mathrm{C}), 128.4$ (2C), 128.3, 128.2 (2C), 127.4, 127.3 (2C), 126.0, 120.3, 109.9, 85.5, 77.4, 75.5, 63.6, 63.5, 62.2, 61.0, 54.7, 33.9, 14.9 ppm. ESI-MS m/z (rel int): (pos) $443.2\left([\mathrm{M}+\mathrm{H}]^{+}, 100\right)$; (neg) 441.2 ([M-H] $]^{-}$ , 100). HRMS (ESI): [M+H] ${ }^{+}$Calculated for: $\mathrm{C}_{28} \mathrm{H}_{31} \mathrm{~N}_{2} \mathrm{O}_{3}{ }^{+}: 443.2329$, found: 443.2333. Absolute difference (ppm): 0.90.

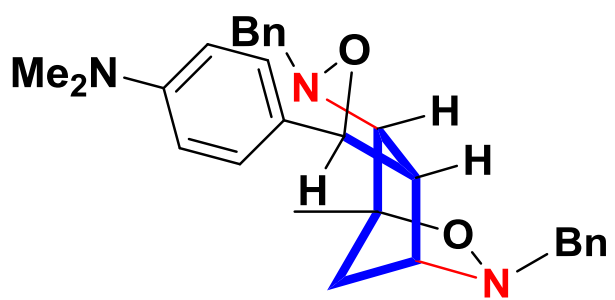

1,5-dibenzyl-7-methylhexahydro-1H-4,7-methanoisoxazolo[4,3-d][1,2]oxazin-3-yl)-N,N-

dimethylaniline (1h): 4-Dimethylaminocinnamaldehyde $(18 \mathrm{mg}, 0.1 \mathrm{mmol})$ was reacted using general method and the resulting BIX was purified by silica gel flash chromatography (10:1 heptanes/EtOAc), yielding $\mathbf{1 h}(38 \mathrm{mg}, 84 \%)$ as a yellow oil. TLC: $R_{f} 0.45$ (2:1 heptanes/EtOAc). IR (thin film): 3030, 2927, 1615, 1524, 1496, 1453, 1354, 1266, 1159, $1028 \mathrm{~cm}^{-1}$. ${ }^{\mathbf{1}} \mathbf{H}-\mathbf{N M R}$ $\left(400 \mathrm{MHz}, \mathrm{CDCl}_{3}\right): \delta 7.42-7.09(\mathrm{~m}, 12 \mathrm{H}), 6.84(\mathrm{~d}, J=8.6 \mathrm{~Hz}, 2 \mathrm{H}), 4.49(\mathrm{~d}, J=6.9 \mathrm{~Hz}, 1 \mathrm{H}), 4.11-$ $3.93(\mathrm{~m}, 3 \mathrm{H}), 3.69(\mathrm{~d}, J=12.8 \mathrm{~Hz}, 1 \mathrm{H}), 3.38(\mathrm{~s}, 1 \mathrm{H}), 3.20(\mathrm{dd}, J=8.4 \mathrm{~Hz}, 1 \mathrm{H}), 3.15(\mathrm{t}, J=7.6$ $\mathrm{Hz}, 1 \mathrm{H}), 2.92(\mathrm{~s}, 6 \mathrm{H}), 2.47(\mathrm{~d}, J=11.2 \mathrm{~Hz}, 1 \mathrm{H}), 1.95(\mathrm{dd}, J=11.2 \mathrm{~Hz}, 1 \mathrm{H}), 1.39(\mathrm{~s}, 3 \mathrm{H}) .{ }^{13} \mathbf{C}-\mathbf{N M R}$ $\left(100 \mathrm{MHz}, \mathrm{CDCl}_{3}\right): \delta 150.5,137.3,136.8,129.8,129.5,129.3,129.2,128.7,128.5,128.4,128.3$, $128.2,128.0,127.3,127.2,126.2,112.2$ (2C), 85.4, 82.1, 75.5, 63.5, 63.0, 62.4, 60.5, $40.4(2 \mathrm{C})$, 34.1, 14.8 ppm. ESI-MS m/z (rel int): (pos) 456.2 ([M+H $\left.]^{+}, 100\right)$; (neg) $454.2\left([\mathrm{M}-\mathrm{H}]^{-}, 100\right)$. 
HRMS (ESI): $[\mathrm{M}+\mathrm{H}]^{+}$Calculated for: $\mathrm{C}_{29} \mathrm{H}_{34} \mathrm{~N}_{3} \mathrm{O}_{2}{ }^{+}$: 456.2646, found: 456.2642. Absolute difference (ppm): 0.88 .

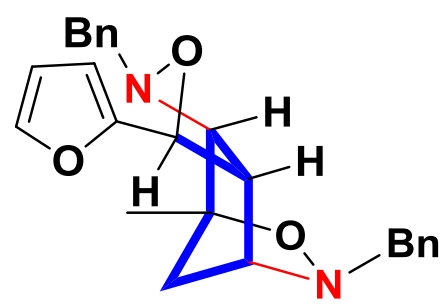

\section{1,5-dibenzyl-3-(furan-2-yl)-7-methylhexahydro-1H-4,7-methanoisoxazolo[4,3-}

d][1,2]oxazine (1i): 3-Furylpropenal $(12 \mathrm{mg}, 0.1 \mathrm{mmol})$ was reacted using general method and the resulting BIX was purified by silica gel flash chromatography (10:1 heptanes/EtOAc), yielding 1i $(29 \mathrm{mg}, 71 \%)$ as a white solid. MP: $112-114^{\circ} \mathrm{C}$ TLC: $R_{f} 0.56$ (2:1 heptanes/EtOAc). IR (thin film): 3058, 2981, 1621, 1530, 1450, 1203, 1142, 1079, $1073 \mathrm{~cm}^{-1} .{ }^{\mathbf{1}} \mathbf{H}-\mathbf{N M R}\left(400 \mathrm{MHz}, \mathrm{CDCl}_{3}\right)$ : $\delta 7.42(\mathrm{~s}, 1 \mathrm{H}), 7.37-7.20(\mathrm{~m}, 10 \mathrm{H}), 6.34(\mathrm{~s}, 2 \mathrm{H}), 4.66(\mathrm{~d}, J=4.6 \mathrm{~Hz}, 1 \mathrm{H}), 4.19-3.98(\mathrm{~m}, 2 \mathrm{H}), 3.81$ $(\mathrm{d}, J=13.2 \mathrm{~Hz}, 1 \mathrm{H}), 3.68(\mathrm{~d}, J=12.8 \mathrm{~Hz}, 1 \mathrm{H}), 3.52(\mathrm{t}, J=6.0 \mathrm{~Hz}, 1 \mathrm{H}), 3.43(\mathrm{~s}, 1 \mathrm{H}), 3.35(\mathrm{~d}, J=$ $7.8 \mathrm{~Hz}, 1 \mathrm{H}), 2.26(\mathrm{~d}, J=11.3 \mathrm{~Hz}, 1 \mathrm{H}), 1.95(\mathrm{~d}, J=11.4 \mathrm{~Hz}, 1 \mathrm{H}), 1.34(\mathrm{~s}, 3 \mathrm{H}) .{ }^{13} \mathbf{C}-\mathbf{N M R}(100$ $\left.\mathrm{MHz}, \mathrm{CDCl}_{3}\right): \delta 152.8,142.8,137.3,136.8,129.4(2 \mathrm{C}), 129.3(2 \mathrm{C}), 128.5(2 \mathrm{C}), 128.2,127.5(2 \mathrm{C})$, 127.4, 110.5, 108.5, 85.6, 76.2, 75.5, 64.1, 63.6, 62.4, 57.1, 34.1, 14.7 ppm. ESI-MS m/z (rel int): (pos) $403.2\left([\mathrm{M}+\mathrm{H}]^{+}, 100\right)$; (neg) $401.2\left([\mathrm{M}-\mathrm{H}]^{-}, 100\right)$. HRMS (ESI): $[\mathrm{M}+\mathrm{H}]^{+}$Calculated for: $\mathrm{C}_{25} \mathrm{H}_{27} \mathrm{~N}_{2} \mathrm{O}_{3}{ }^{+}$: 403.2016, found: 403.2024. Absolute difference (ppm): 1.98 .

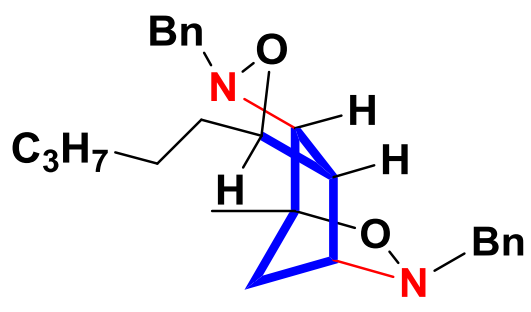

1,5-dibenzyl-3,7-dimethyl-3-pentylhexahydro-1H-4,7-methanoisoxazolo[4,3-d][1,2]oxazine (1j): Trans-2-octenal (15 mg, $0.1 \mathrm{mmol})$ was reacted using general method and the resulting BIX was purified by silica gel flash chromatography (10:1 heptanes/EtOAc), yielding $\mathbf{1 j}$ (29 mg, 71\%) as a light yellow oil. TLC: $R_{f} 0.63$ (2:1 heptanes/EtOAc). IR (thin film): 3030, 2929, 1605, 1496, 1453, 1378, 1208, 1081, $976 \mathrm{~cm}^{-1} .{ }^{1} \mathbf{H}-\mathbf{N M R}\left(400 \mathrm{MHz}, \mathrm{CDCl}_{3}\right): \delta 7.46-7.15(\mathrm{~m}, 10 \mathrm{H}), 4.16-3.82$ $(\mathrm{m}, 3 \mathrm{H}), 3.69(\mathrm{~d}, J=12.8 \mathrm{~Hz}, 1 \mathrm{H}), 3.54(\mathrm{dd}, J=6.6,6.6 \mathrm{~Hz}, 1 \mathrm{H}), 3.27(\mathrm{~s}, 1 \mathrm{H}), 3.05(\mathrm{dd}, J=8.2$, $1.6 \mathrm{~Hz}, 1 \mathrm{H}), 2.69(\mathrm{t}, J=7.4 \mathrm{~Hz}, 1 \mathrm{H}), 2.27(\mathrm{~d}, J=11.2 \mathrm{~Hz}, 1 \mathrm{H}), 1.86(\mathrm{dd}, J=11.2,2.1 \mathrm{~Hz}, 1 \mathrm{H})$, $1.68-1.60(\mathrm{~m}, 1 \mathrm{H}), 1.58-1.48(\mathrm{~m}, 1 \mathrm{H}), 1.34(\mathrm{~s}, 3 \mathrm{H}), 1.28-1.22(\mathrm{~m}, 6 \mathrm{H}), 0.83(\mathrm{t}, J=6.5 \mathrm{~Hz}, 3 \mathrm{H})$. ${ }^{13}$ C-NMR (100 MHz, $\left.\mathrm{CDCl}_{3}\right): \delta 137.6,137.0,129.7,129.2,129.1$ (2C), 128.5, 128.3 (2C), 128.2, 
127.4, 127.3, 85.4, 80.7, 75.15, 63.7, 63.6, 62.2, 59.7, 34.7, 34.1, 31.7, 25.9, 22.5, 14.8, 13.9 ppm. ESI-MS $m / z$ (rel int): (pos) $407.3\left([\mathrm{M}+\mathrm{H}]^{+}, 100\right)$; (neg) 405.3 ([M-H] $\left.]^{-}, 100\right)$. HRMS (ESI): $[\mathrm{M}+\mathrm{H}]^{+}$Calculated for: $\mathrm{C}_{26} \mathrm{H}_{35} \mathrm{~N}_{2} \mathrm{O}_{2}{ }^{+}:$407.2693, found: 407.2688. Absolute difference (ppm): 1.23 .

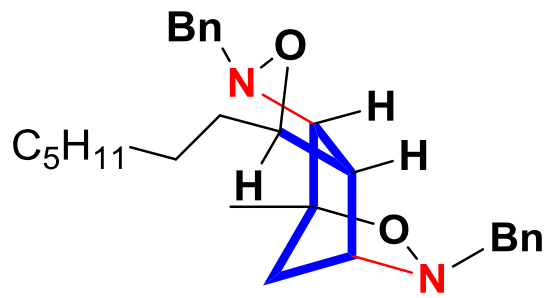

1,5-dibenzyl-3-heptyl-7-methylhexahydro-1H-4,7-methanoisoxazolo[4,3-d][1,2]oxazine (1k): Trans-2-decenal (16 mg, $0.1 \mathrm{mmol})$ was reacted using general method and the resulting BIX was purified by silica gel flash chromatography (10:1 heptanes/EtOAc), yielding $1 \mathbf{k}(32 \mathrm{mg}, 75 \%)$ as a light yellow oil. TLC: $R_{f} 0.64$ (2:1 heptanes/EtOAc). IR (thin film): 3023, 2972, 1510, 1450, $1385,1265,1065 \mathrm{~cm}^{-1} .{ }^{1} \mathbf{H}-\mathbf{N M R}\left(400 \mathrm{MHz} \mathrm{CDCl}_{3}\right): \delta$ 7.54-6.95 (m ,10H), 4.20-3.81 (m, 3H), $3.69(\mathrm{~d}, J=12.9 \mathrm{~Hz}, 1 \mathrm{H}), 3.53(\mathrm{dd}, J=6.6,6.6 \mathrm{~Hz}, 1 \mathrm{H}), 3.27(\mathrm{~s}, 1 \mathrm{H}), 3.05(\mathrm{dd}, J=8.2,1.6 \mathrm{~Hz}$, $1 \mathrm{H}), 2.69(\mathrm{t}, J=7.4 \mathrm{~Hz}, 1 \mathrm{H}), 2.27(\mathrm{~d}, J=11.2 \mathrm{~Hz}, 1 \mathrm{H}), 1.86(\mathrm{dd}, J=11.2,2.0 \mathrm{~Hz}, 1 \mathrm{H}), 1.69-1.61$ $(\mathrm{m}, 1 \mathrm{H}), 1.58(\mathrm{~s}, 3 \mathrm{H}), 1.57-1.50(\mathrm{~m}, 1 \mathrm{H}), 1.28-1.22(\mathrm{~m}, 10 \mathrm{H}), 0.87(\mathrm{t}, J=6.5 \mathrm{~Hz}, 3 \mathrm{H}) .{ }^{13} \mathbf{C}-\mathbf{N M R}$ $\left(100 \mathrm{MHz}, \mathrm{CDCl}_{3}\right): \delta 137.6,137.0,129.2(2 \mathrm{C}), 129.1$ (2C), 128.5, 128.3 (2C), 128.2, 127.4, 127.3, 85.4, 80.8, 75.2, 63.6, 63.5, 62.3, 59.7, 34.7, 34.0, 31.9, 31.7, 29.1, 26.3, 22.6, 14.8, 14.1 ppm. ESI-MS $m / z$ (rel int): (pos) $435.3\left([\mathrm{M}+\mathrm{H}]^{+}, 100\right)$; (neg) 433.3 .0 ([M-H $\left.]^{-}, 100\right)$. HRMS (ESI): $[\mathrm{M}+\mathrm{H}]^{+}$Calculated for: $\mathrm{C}_{28} \mathrm{H}_{39} \mathrm{~N}_{2} \mathrm{O}_{2}{ }^{+}:$435.3006, found: 435.3013. Absolute difference (ppm): 1.61 .

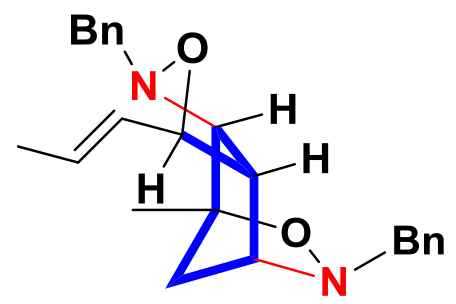

1,5-dibenzyl-7-methyl-3-((E)-prop-1-en-1-yl)hexahydro-1H-4,7-methanoisoxazolo[4,3d][1,2] oxazine (11): Trans-trans-2,4-hexadienal $(9.6 \mathrm{mg}, 0.1 \mathrm{mmol})$ was reacted using general method and the resulting BIX was purified by silica gel flash chromatography (10:1 heptanes/EtOAc), yielding 11 (30 mg, 80\%) as a light yellow oil. TLC: $R_{f} 0.60$ (2:1 heptanes/EtOAc). IR (thin film): 3032, 2930, 1496, 1453, 1379, 1265, 1136, 1080, $1028 \mathrm{~cm}^{-1}$. ${ }^{1}$ H-NMR $\left(400 \mathrm{MHz}, \mathrm{CDCl}_{3}\right): \delta 7.50-6.92(\mathrm{~m}, 10 \mathrm{H}), 5.68(\mathrm{dq}, J=10.6,7.1 \mathrm{~Hz}, 1 \mathrm{H}), 5.48(\mathrm{dd}, J=$ 10.6, 6.4 Hz, 1H), 4.14-3.97 (m, 3H), 3.89 (d, $J=13.0 \mathrm{~Hz}, 1 \mathrm{H}), 3.69$ (d, J=12.9 Hz, 1H), 3.34 (s, $1 \mathrm{H}), 3.10(\mathrm{~d}, J=7.8 \mathrm{~Hz}, 1 \mathrm{H}), 2.84(\mathrm{t}, J=8.0 \mathrm{~Hz}, 1 \mathrm{H}), 2.28(\mathrm{~d}, J=12.5 \mathrm{~Hz}, 1 \mathrm{H}), 1.90(\mathrm{~d}, J=12.5$ 
$\mathrm{Hz}, 1 \mathrm{H}), 1.68(\mathrm{~d}, J=7.1 \mathrm{~Hz}, 3 \mathrm{H}), 1.58(\mathrm{~s}, 3 \mathrm{H}) \cdot{ }^{13} \mathrm{C}-\mathbf{N M R}\left(100 \mathrm{MHz}, \mathrm{CDCl}_{3}\right): \delta 137.5,136.9$, 130.6 (2C), 129.7 (2C), 129.3 (2C), 129.1 (2C), 128.5, 128.3, 127.4, 127.3, 85.4, 82.1, 75.6, 63.7, 63.4, 62.4, 59.4, 34.1, 17.8, 14.8 ppm. ESI-MS m/z (rel int): (pos) 377.2 ([M+H] $\left.]^{+}, 100\right)$; (neg) $375.2\left([\mathrm{M}-\mathrm{H}]^{-}, 100\right)$. HRMS (ESI): $[\mathrm{M}+\mathrm{H}]^{+}$Calculated for: $\mathrm{C}_{24} \mathrm{H}_{29} \mathrm{~N}_{2} \mathrm{O}_{2}{ }^{+}$: 377.2224 , found: 377.2229. Absolute difference (ppm): 1.33.

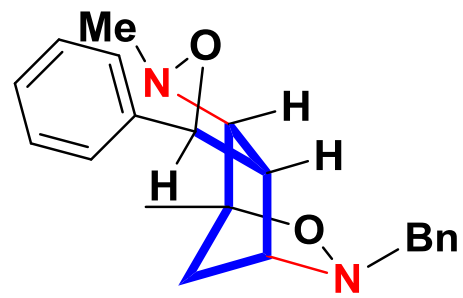

\section{5-benzyl-1,7-dimethyl-3-phenylhexahydro-1H-4,7-methanoisoxazolo[4,3-d][1,2] oxazine} (1 m): Cinnamaldehyde $(13 \mathrm{mg}, 0.1 \mathrm{mmol})$ was reacted using general method and the resulting BIX was purified by silica gel flash chromatography (10:1 heptanes/EtOAc), yielding $1 \mathbf{m}(28 \mathrm{mg}$, 82\%) as a colorless oil. TLC: $R_{f} 0.40$ (2:1 heptanes/EtOAc). IR (thin film): 3033, 2935, 1496, 1453, 1380, 1265, 1207, 1130, 1107, 1081, $1053 \mathrm{~cm}^{-1} .{ }^{\mathbf{1}} \mathbf{H}-\mathbf{N M R}\left(400 \mathrm{MHz}, \mathrm{CDCl}_{3}\right): \delta 7.49-6.95$ $(\mathrm{m}, 10 \mathrm{H}), 4.52(\mathrm{~d}, J=7.8 \mathrm{~Hz}, 1 \mathrm{H}), 4.04(\mathrm{~d}, J=12.9 \mathrm{~Hz}, 1 \mathrm{H}), 3.72(\mathrm{~d}, J=12.9 \mathrm{~Hz}, 1 \mathrm{H}), 3.41(\mathrm{~s}$, $1 \mathrm{H}), 3.11(\mathrm{t}, J=8.1 \mathrm{~Hz}, 1 \mathrm{H}), 2.92(\mathrm{dd}, J=8.4,1.6 \mathrm{~Hz}, 1 \mathrm{H}), 2.78(\mathrm{~s}, 3 \mathrm{H}), 2.46(\mathrm{t}, J=11.4 \mathrm{~Hz}, 1 \mathrm{H})$, $1.98(\mathrm{~d}, J=11.4 \mathrm{~Hz}, 1 \mathrm{H}), 1.47(\mathrm{~s}, 3 \mathrm{H}) .{ }^{13} \mathbf{C}-\mathbf{N M R}\left(100 \mathrm{MHz}, \mathrm{CDCl}_{3}\right): \delta 138.5,136.8,129.2,128.7$, 128.6, 128.5, 128.4, 128.3 (2C), 128.2, 127.4, 127.0, 85.2, 82.1, 78.5, 63.2, 62.4, 61.9, 46.0, 34.0, 14.7 ppm. ESI-MS m/z (rel int): (pos) 337.2 ([M+H] $\left.]^{+}, 100\right)$; (neg) $335.2\left([\mathrm{M}-\mathrm{H}]^{-}, 100\right)$. HRMS (ESI): $[\mathrm{M}+\mathrm{H}]^{+}$Calculated for: $\mathrm{C}_{21} \mathrm{H}_{25} \mathrm{~N}_{2} \mathrm{O}_{2}{ }^{+}: 337.1911$, found: 337.1915. Absolute difference (ppm): 1.19 .

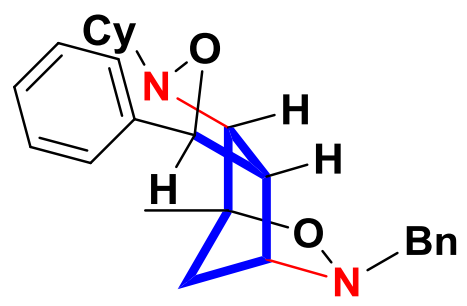

5-benzyl-1-cyclohexyl-7-methyl-3-phenylhexahydro-1H-4,7-methanoisoxazolo[4,3-

d][1,2]oxazine (1n): Cinnamaldehyde $(13 \mathrm{mg}, 0.1 \mathrm{mmol})$ was reacted using general method and the resulting BIX was purified by silica gel flash chromatography (10:1 heptanes/EtOAc), yielding 1n $(32 \mathrm{mg}, 79 \%)$ as a white solid. MP: $160-162^{\circ} \mathrm{C}$. TLC: $R_{f} 0.65$ (2:1 heptanes/EtOAc). IR (thin film): 3053, 2933, 1496, 1451, 1351, 1264, 1084, $1028 \mathrm{~cm}^{-1} .{ }^{\mathbf{1}} \mathbf{H}-\mathbf{N M R}\left(400 \mathrm{MHz}, \mathrm{CDCl}_{3}\right): \delta 7.47-$ $7.15(\mathrm{~m}, 10 \mathrm{H}), 4.53(\mathrm{~d}, J=6.4 \mathrm{~Hz}, 1 \mathrm{H}), 4.03(\mathrm{~d}, J=12.9 \mathrm{~Hz}, 1 \mathrm{H}), 3.71(\mathrm{~d}, J=12.9 \mathrm{~Hz}, 1 \mathrm{H}), 3.49-$ $3.33(\mathrm{~m}, 2 \mathrm{H}), 3.15(\mathrm{t}, J=7.4 \mathrm{~Hz}, 1 \mathrm{H}), 2.51(\mathrm{tt}, J=11.2,3.5 \mathrm{~Hz}, 1 \mathrm{H}), 2.40(\mathrm{~d}, J=11.3 \mathrm{~Hz}, 1 \mathrm{H})$, $1.98-2.04(\mathrm{~m}, 1 \mathrm{H}), 1.95(\mathrm{~d}, J=11.3,1.8 \mathrm{~Hz}, 1 \mathrm{H}), 1.86-1,69(\mathrm{~m}, 3 \mathrm{H}), 1.45(\mathrm{~s}, 3 \mathrm{H}), 1.42-1.32(\mathrm{~m}$ 
2H), 1.25-0.98 (m, 4H). ${ }^{13} \mathrm{C}-\mathbf{N M R}\left(100 \mathrm{MHz}, \mathrm{CDCl}_{3}\right): \delta 140.0,136.9,129.3,128.5(2 \mathrm{C}), 128.4$, 128.0, (2C), 127.4, (2C), 127.0 (2C), 85.9, 82.0, 72.2, 65.3, 63.9, 62.4, 60.5, 34.1, 32.5, 27.1, 26.1, 25.7, 25.3, 15.2 ppm. ESI-MS m/z (rel int): (pos) $405.3\left([\mathrm{M}+\mathrm{H}]^{+}, 100\right)$; (neg) 403.3 ([M-H] $]^{-}$, 100). HRMS (ESI): $[\mathrm{M}+\mathrm{H}]^{+}$Calculated for: $\mathrm{C}_{26} \mathrm{H}_{33} \mathrm{~N}_{2} \mathrm{O}_{2}{ }^{+}:$405.2537, found: 405.2543. Absolute difference (ppm): 1.48 .

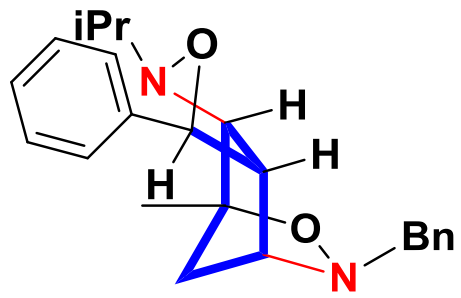

\section{5-benzyl-1-isopropyl-7-methyl-3-phenylhexahydro-1H-4,7-methanoisoxazolo[4,3-}

d][1,2] oxazine (10): Cinnamaldehyde (13 mg, $0.1 \mathrm{mmol})$ was reacted using general method and the resulting BIX was purified by silica gel flash chromatography (10:1 heptanes/EtOAc), yielding 10 (31 mg, 84\%) as a light yellow oil. TLC: $R_{f} 0.52$ (2:1 heptanes/EtOAc). IR (thin film): 3031, 2971, 1605, 1496, 1450, 1379, 1289, 1202, 1056, $1029 \mathrm{~cm}^{-1} .{ }^{\mathbf{1}} \mathbf{H}-\mathbf{N M R}\left(400 \mathrm{MHz}, \mathrm{CDCl}_{3}\right): \delta 7.39-$ $7.18(\mathrm{~m}, 10 \mathrm{H}), 4.53(\mathrm{~d}, J=6.4 \mathrm{~Hz}, 1 \mathrm{H}), 4.03(\mathrm{~d}, J=12.9 \mathrm{~Hz}, 1 \mathrm{H}), 3.71(\mathrm{~d}, J=12.9 \mathrm{~Hz}, 1 \mathrm{H}), 3.42$ (s, 1H), 3.35 (dd, $J=8.4,1.6 \mathrm{~Hz}, 1 \mathrm{H}), 3.14$ (t, $J=7.4 \mathrm{~Hz}, 1 \mathrm{H}), 2.93$ (qq, $J=7.3,7.3 \mathrm{~Hz}, 1 \mathrm{H}), 2.42$ $(\mathrm{d}, J=11.3 \mathrm{~Hz}, 1 \mathrm{H}), 1.96(\mathrm{~d}, J=11.3,1.9 \mathrm{~Hz}, 1 \mathrm{H}), 1.46(\mathrm{~s}, 3 \mathrm{H}), 1.96(\mathrm{dd}, J=7.3,7.3 \mathrm{~Hz}, 6 \mathrm{H})$. ${ }^{13}$ C-NMR (100 MHz, $\mathrm{CDCl}_{3}$ ): $\delta 140.0,136.9,129.3,128.5$ (2C), 128.4, 128.0, (2C), 127.4, (2C), 127.0, 126.9, 85.9, 81.9, 72.6, 63.9, 62.5, 60.7, 56.6, 34.1, 22.7, 22.0, $15.2 \mathrm{ppm}$. ESI-MS $\mathrm{m} / z$ (rel int): (pos) $365.2\left([\mathrm{M}+\mathrm{H}]^{+}, 100\right)$; (neg) 363.2 ([M-H] $\left.]^{-}, 100\right)$. HRMS (ESI): $[\mathrm{M}+\mathrm{H}]^{+}$Calculated for: $\mathrm{C}_{23} \mathrm{H}_{29} \mathrm{~N}_{2} \mathrm{O}_{2}{ }^{+}: 365.2224$, found: 365.2231 . Absolute difference (ppm): 1.92 .

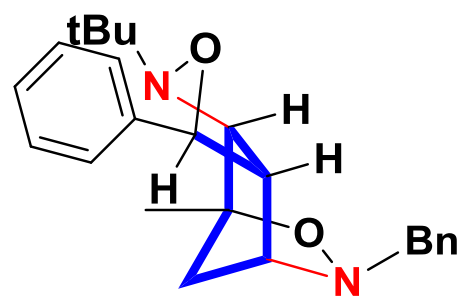

5-benzyl-1-isopropyl-7-methyl-3-phenylhexahydro-1H-4,7-methanoisoxazolo[4,3-

d][1,2] oxazine (1p): Cinnamaldehyde (13 mg, $0.1 \mathrm{mmol})$ was reacted using general method and the resulting BIX was purified by silica gel flash chromatography (10:1 heptanes/EtOAc), yielding 
$1 p(28 \mathrm{mg}, 73 \%)$ as a white solid. MP: $129-131^{\circ} \mathrm{C}$. TLC: $R_{f} 0.52(2: 1$ heptanes/EtOAc). IR (thin film): $3035,2981,1510,1455,1324,1255,1106,1070 \mathrm{~cm}^{-1} .{ }^{1} \mathbf{H}-\mathbf{N M R}\left(400 \mathrm{MHz}, \mathrm{CDCl}_{3}\right): \delta 7.44-$ $7.14(\mathrm{~m}, 10 \mathrm{H}), 4.54(\mathrm{~d}, J=7.5 \mathrm{~Hz}, 1 \mathrm{H}), 4.02(\mathrm{~d}, J=12.9 \mathrm{~Hz}, 1 \mathrm{H}), 3.72(\mathrm{~d}, J=12.9 \mathrm{~Hz}, 1 \mathrm{H}), 3.44-$ $3.40(\mathrm{~m}, 2 \mathrm{H}), 3.14(\mathrm{t}, J=8.4 \mathrm{~Hz}, 1 \mathrm{H}), 2.39(\mathrm{~d}, J=11.5 \mathrm{~Hz}, 1 \mathrm{H}), 1.97(\mathrm{~d}, J=11.6 \mathrm{~Hz}, 1 \mathrm{H}), 1.49$ $(\mathrm{s}, 3 \mathrm{H}), 1.13(\mathrm{~s}, 9 \mathrm{H}) .{ }^{13} \mathrm{C}-\mathbf{N M R}\left(100 \mathrm{MHz}, \mathrm{CDCl}_{3}\right): \delta 139.9,136.8,129.3(2 \mathrm{C}), 128.5(2 \mathrm{C}), 128.0$ (2C), 127.4 (2C), 126.8 (2C), 86.2, 81.6, 69.9, 63.9, 62.7, 62.0, 59.5, 34.8, 26.6, 15.3 ppm. ESI-MS $\mathrm{m} / \mathrm{z}$ (rel int): (pos) $379.2\left([\mathrm{M}+\mathrm{H}]^{+}, 100\right)$; (neg) $377.2\left([\mathrm{M}-\mathrm{H}]^{-}, 100\right)$. HRMS (ESI): $[\mathrm{M}+\mathrm{H}]^{+}$ Calculated for: $\mathrm{C}_{24} \mathrm{H}_{31} \mathrm{~N}_{2} \mathrm{O}_{2}{ }^{+}: 379.2380$, found: 379.2386 . Absolute difference (ppm): 1.58 .

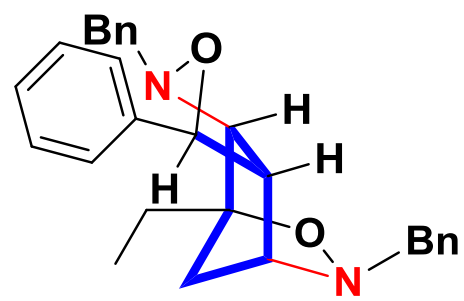

1,5-dibenzyl-7-ethyl-3-phenylhexahydro-1H-4,7-methanoisoxazolo[4,3-d][1,2] ]oxazine (1q): Cinnamaldehyde (13 mg, $0.1 \mathrm{mmol})$ was reacted using general method and the resulting BIX was purified by silica gel flash chromatography (10:1 heptanes/EtOAc), yielding 1q $(29 \mathrm{mg}, 68 \%)$ as a white solid. MP: $136-139^{\circ} \mathrm{C}$. TLC: $R_{f} 0.63$ (2:1 heptanes/EtOAc). IR (thin film): 3032,2974 , 1605, 1496, 1453, 1208, 1156, 1059, $1028 \mathrm{~cm}^{-1} .{ }^{\mathbf{1}} \mathbf{H}-\mathbf{N M R}\left(400 \mathrm{MHz}, \mathrm{CDCl}_{3}\right): \delta$ 7.43-7.14 (m, $15 \mathrm{H}), 4.57$ (d, $J=6.9 \mathrm{~Hz}, 1 \mathrm{H}), 4.11-3.83(\mathrm{~m}, 3 \mathrm{H}), 3.69$ (d, $J=12.8 \mathrm{~Hz}, 1 \mathrm{H}), 3.45$ (s, 1H), 3.27 $(\mathrm{dd}, J=8.4,1.5 \mathrm{~Hz}, 1 \mathrm{H}), 3.16(\mathrm{t}, J=7.5 \mathrm{~Hz}, 1 \mathrm{H}), 2.34(\mathrm{~d}, J=11.3 \mathrm{~Hz}, 1 \mathrm{H}), 2.09$ (dd, $J=11.5$, $2.0 \mathrm{~Hz}, 1 \mathrm{H}), 1.90(\mathrm{dq}, J=11.0,7.3 \mathrm{~Hz}, 1 \mathrm{H}), 1.75(\mathrm{dd}, J=11.2,7.3 \mathrm{~Hz}, 1 \mathrm{H}), 1.04$ (t, $J=7.3 \mathrm{~Hz}$, 3H). ${ }^{13} \mathrm{C}-\mathrm{NMR}\left(100 \mathrm{MHz}, \mathrm{CDCl}_{3}\right): \delta 139.5,137.4,136.9,129.3(2 \mathrm{C}), 129.1,128.5(2 \mathrm{C}), 128.4$ (2C), 128.2, 128.0 (2C), 127.4 (2C), 127.3, 126.8 (2C), 88.9, 82.2, 74.8, 63.5, 63.0, 62.2, 60.8, 30.9, 21.3, 8.8 ppm. ESI-MS m/z (rel int): (pos) 427.2 ([M+H $\left.]^{+}, 100\right)$; (neg) $425.2\left([\mathrm{M}-\mathrm{H}]^{-}, 100\right)$. HRMS (ESI): $[\mathrm{M}+\mathrm{H}]^{+}$Calculated for: $\mathrm{C}_{28} \mathrm{H}_{31} \mathrm{~N}_{2} \mathrm{O}_{2}{ }^{+}:$427.2380, found: 427.2386. Absolute difference (ppm): 1.40 . 


\section{SYNTHESIS OF CARBACYCLES FROM TABLES 1 AND 2:}

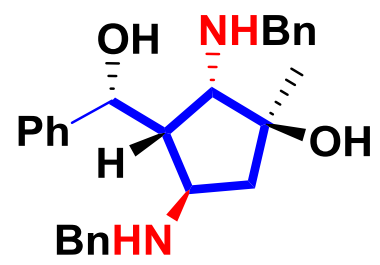

2,4-bis(benzylamino)-3-(hydroxy(phenyl)methyl)-1-methylcyclopentan-1-ol (2a): BIX 1a reacted under reductive cleavage conditions (general method) and after extraction with pentanes, CB 2 a was obtained as a white solid $(10 \mathrm{mg}, 96 \%)$. MP: $147-148^{\circ} \mathrm{C}$. TLC: $R_{f}: 0.11$ (1:1 heptanes/EtOAc). IR (thin film) 3267, 3221, 3032, 2930, 1604, 1496, 1453, 1363, $1266 \mathrm{~cm}^{-1}$. ${ }^{1}$ H-NMR $\left(400 \mathrm{MHz}, \mathrm{CDCl}_{3}\right): \delta$ 7.46-7.37 (m 2H), 7.35-7.21 (m, 11H), 7.15-7.03 (m, 2H), 4.42 $(\mathrm{d}, J=8.2 \mathrm{~Hz}, 1 \mathrm{H}), 4.23(\mathrm{~d}, J=14.7 \mathrm{~Hz}, 1 \mathrm{H}), 4.01(\mathrm{~d}, J=14.7 \mathrm{~Hz}, 1 \mathrm{H}), 3.72(\mathrm{~d}, J=13.1 \mathrm{~Hz}, 1 \mathrm{H})$, $3.60(\mathrm{~d}, J=13.1 \mathrm{~Hz}, 1 \mathrm{H}), 3.48(\mathrm{dd}, J=8.4,1.6 \mathrm{~Hz}, 1 \mathrm{H}), 2.94(\mathrm{td}, J=8.4,1.7 \mathrm{~Hz}, 1 \mathrm{H}), 2.28(\mathrm{dd}$, $J=13.9,5.0 \mathrm{~Hz}, 1 \mathrm{H}), 1.88(\mathrm{dd}, J=13.9,1.8 \mathrm{~Hz}, 1 \mathrm{H}), 1.43(\mathrm{~s}, 3 \mathrm{H}) .{ }^{13} \mathbf{C}-\mathbf{N M R}\left(100 \mathrm{MHz}, \mathrm{CDCl}_{3}\right)$ : $\delta 138.9,138.4,137.8,129.0(2 \mathrm{C}), 128.6(2 \mathrm{C}), 128.5$ (2C), 128.3 (2C), 128.2 (2C), $127.4(2 \mathrm{C})$, 127.2, 126.8, 126.5, 83.3, 81.1, 80.9, 63.8, 62.6, 60.5, 51.2, 41.3, 21.5 ppm. ESI-MS m/z (rel int): (pos) $417.3\left([\mathrm{M}+\mathrm{H}]^{+}, 100\right)$; (neg) $415.3\left([\mathrm{M}-\mathrm{H}]^{-}, 100\right)$. HRMS (ESI): $[\mathrm{M}+\mathrm{H}]^{+} \mathrm{Calculated}$ for: $\mathrm{C}_{27} \mathrm{H}_{33} \mathrm{~N}_{2} \mathrm{O}_{2}{ }^{+}: 417.2537$, found: 417.2531. Absolute difference (ppm): 1.44.<smiles>C[C@]1(O)C[C@@H](N)[C@H]([C@H](O)c2ccc(Br)cc2)[C@@H]1Br</smiles>

2,4-bis(benzylamino)-3-((4-bromophenyl)(hydroxy)methyl)-1-methylcyclopentan-1-ol (2b): BIX 1b reacted under reductive cleavage conditions (general method) and after extraction with pentanes, CB $2 \mathbf{b}$ was obtained as a white solid (9 mg, 96\%). MP: $138-141^{\circ} \mathrm{C}$. TLC: $R_{f}: 0.05(1: 1$ heptanes/EtOAc). IR (thin film) 3282, 3243, 3031, 2928, 1604, 1454, 1265, $1160 \mathrm{~cm}^{-1}$. ${ }^{\mathbf{1}} \mathbf{H}-\mathbf{N M R}$ $\left(400 \mathrm{MHz}, \mathrm{CDCl}_{3}\right): \delta 7.49-6.9(\mathrm{~m} \mathrm{14H}), 4.36(\mathrm{~d}, J=8.2 \mathrm{~Hz}, 1 \mathrm{H}), 4.23(\mathrm{~d}, J=14.7 \mathrm{~Hz}, 1 \mathrm{H}), 3.98$ $(\mathrm{d}, J=14.7 \mathrm{~Hz}, 1 \mathrm{H}), 3.72(\mathrm{~d}, J=13.3 \mathrm{~Hz}, 1 \mathrm{H}), 3.60(\mathrm{~d}, J=13.2 \mathrm{~Hz}, 1 \mathrm{H}), 3.47(\mathrm{dd}, J=8.5 \mathrm{~Hz}$, $1 \mathrm{H}), 3.25(\mathrm{~d}, J=4.7 \mathrm{~Hz}, 1 \mathrm{H}), 2.85(\mathrm{t}, J=8.4 \mathrm{~Hz}, 1 \mathrm{H}), 2.26(\mathrm{dd}, J=13.9,4.8 \mathrm{~Hz}, 1 \mathrm{H}), 1.86(\mathrm{~d}, J$ $=13.9 \mathrm{~Hz}, 1 \mathrm{H}), 1.43(\mathrm{~s}, 3 \mathrm{H}) .{ }^{13} \mathbf{C}-\mathbf{N M R}\left(100 \mathrm{MHz}, \mathrm{CDCl}_{3}\right): \delta 138.5,138.1,137.7,131.7,128.9$ (2C), 128.8, 128.6 (2C), 128.4, 128.3, 128.2 (2C), 128.1 (2C), 127.4, 127.3, 122.1, 82.6, 81.1, 80.9, 63.9, 62.6, 60.4, 51.2, 41.4, 21.4 ppm. ESI-MS $\mathrm{m} / z$ (rel int): (pos) $495.2\left([\mathrm{M}+\mathrm{H}]^{+}, 100\right.$ ); (neg) $493.2\left([\mathrm{M}-\mathrm{H}]^{-}, 100\right)$. HRMS (ESI): $[\mathrm{M}+\mathrm{H}]^{+}$Calculated for: $\mathrm{C}_{27} \mathrm{H}_{32} \mathrm{BrN}_{2} \mathrm{O}_{2}{ }^{+}$: 495.1642 , found: 495.1647. Absolute difference (ppm): 1.01. 


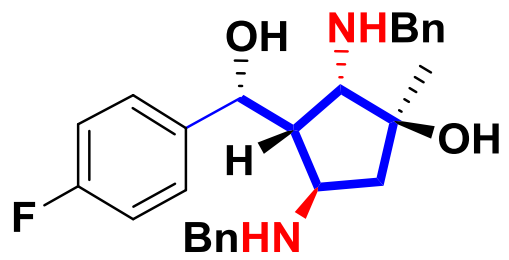

2,4-bis(benzylamino)-3-((4-fluorophenyl)(hydroxy)methyl)-1-methylcyclopentan-1-ol (2c): BIX 1c reacted under reductive cleavage conditions (general method) and after extraction with pentanes, CB 2c was obtained as a white solid $(9 \mathrm{mg}, 86 \%)$. MP: $117-120^{\circ} \mathrm{C}$. TLC: $R_{f}: 0.03(1: 1$ heptanes/EtOAc). IR (thin film) 3223, 3218, 3018, 2927, 1605, 1511, 1454, 1265, $1225 \mathrm{~cm}^{-1}$. ${ }^{1}$ H-NMR (400 MHz, $\left.\mathrm{CDCl}_{3}\right): \delta$ 7.49-6.5 (m 14H), $4.39(\mathrm{~d}, J=8.2 \mathrm{~Hz}, 1 \mathrm{H}), 4.23(\mathrm{~d}, J=14.7 \mathrm{~Hz}$, $1 \mathrm{H}), 3.99(\mathrm{~d}, J=14.7 \mathrm{~Hz}, 1 \mathrm{H}), 3.71(\mathrm{~d}, J=13.1 \mathrm{~Hz}, 1 \mathrm{H}), 3.61(\mathrm{~d}, J=13.2 \mathrm{~Hz}, 1 \mathrm{H}), 3.47(\mathrm{~d}, J=$ $8.5 \mathrm{~Hz}, 1 \mathrm{H}), 3.24(\mathrm{~d}, J=4.7 \mathrm{~Hz}, 1 \mathrm{H}), 2.85(\mathrm{t}, J=8.5 \mathrm{~Hz}, 1 \mathrm{H}), 2.27(\mathrm{dd}, J=14.0,4.8 \mathrm{~Hz}, 1 \mathrm{H}), 1.86$ $(\mathrm{d}, J=13.7 \mathrm{~Hz}, 1 \mathrm{H}), 1.42(\mathrm{~s}, 3 \mathrm{H}) .{ }^{13} \mathrm{C}-\mathbf{N M R}\left(100 \mathrm{MHz}, \mathrm{CDCl}_{3}\right): \delta 138.5,137.7,134.7,128.9$, 128.8, 128.6, 128.5 (2C), 128.3, 128.2 (2C), 128.1, 128.0 (2C), 127.4, 127.2, 115.6, 115.4, 82.6, 81.1, 80.9, 63.8, 62.6, 60.4, 51.2, 41.4, 21.4 ppm. ESI-MS m/z (rel int): (pos) $435.2\left([\mathrm{M}+\mathrm{H}]^{+}\right.$, 100); (neg) $433.2\left([\mathrm{M}-\mathrm{H}]^{-}, 100\right)$. HRMS (ESI): $[\mathrm{M}+\mathrm{H}]^{+}$Calculated for: $\mathrm{C}_{27} \mathrm{H}_{32} \mathrm{FN}_{2} \mathrm{O}_{2}{ }^{+}$: 435.2442 , found: 435.2447. Absolute difference (ppm): 1.15.

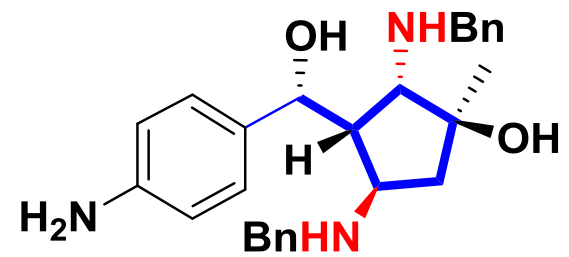

2,4-bis(benzylamino)-3-(hydroxy(4-nitrophenyl)methyl)-1-methylcyclopentan-1-ol (2d): BIX 1d reacted under reductive cleavage conditions (general method) and after extraction with pentanes, CB 2d was obtained as a light yellow solid (12 mg, 92\%). MP: $61-64^{\circ} \mathrm{C}$. TLC: $R_{f}: 0.10$ (1:1 heptanes/EtOAc). IR (thin film) 3298, 3243, 3054, 2918, 1422, 1363, 1266, $1180 \mathrm{~cm}^{-1}$. ${ }^{1} \mathbf{H}-\mathbf{N M R}\left(400 \mathrm{MHz}, \mathrm{CDCl}_{3}\right): \delta$ 7.39-7.16 (m 9H), $7.09(\mathrm{~d}, J=7.7 \mathrm{~Hz}, 1 \mathrm{H}), 6.95-6.91(\mathrm{~m}, 2 \mathrm{H})$, $6.63(\mathrm{dd}, J=11.1,7.8 \mathrm{~Hz}, 2 \mathrm{H}), 4.61(\mathrm{~d}, J=10.5 \mathrm{~Hz}, 1 \mathrm{H}), 4.11(\mathrm{~d}, J=12.4 \mathrm{~Hz}, 1 \mathrm{H}), 3.88(\mathrm{~d}, J=$ $12.6 \mathrm{~Hz}, 1 \mathrm{H}), 3.46(\mathrm{~d}, J=8.7 \mathrm{~Hz}, 1 \mathrm{H}), 3.37-3.22(\mathrm{~m}, 2 \mathrm{H}), 3.11(\mathrm{~d}, J=9.7 \mathrm{~Hz}, 1 \mathrm{H}), 2.84(\mathrm{~s}, 1 \mathrm{H})$, $1.84(\mathrm{~d}, J=6.5 \mathrm{~Hz}, 1 \mathrm{H}), 1.69$ (d, $J=13.6 \mathrm{~Hz}, 1 \mathrm{H}), 1.47(\mathrm{~s}, 3 \mathrm{H}) .{ }^{13} \mathbf{C}-\mathbf{N M R}\left(100 \mathrm{MHz}, \mathrm{CDCl}_{3}\right): \delta$ 146.2, 139.4, 129.2, 128.8 (4C), 128.4 (4C), 128.1 (2C), 127.6, 127.0, 124.5, 117.5, 116.7, 82.4, 76.4, 69.7, 58.9, 54.1, 51.3, 49.5, 43.1, 22.7 ppm. ESI-MS m/z (rel int): (pos) $432.2\left([\mathrm{M}+\mathrm{H}]^{+}\right.$, 100); (neg) $430.2\left([\mathrm{M}-\mathrm{H}]^{-}, 100\right)$. HRMS (ESI): $[\mathrm{M}+\mathrm{H}]^{+}$Calculated for: $\mathrm{C}_{27} \mathrm{H}_{34} \mathrm{~N}_{3} \mathrm{O}_{2}{ }^{+}: 432.2646$, found: 432.2651. Absolute difference (ppm): 1.16. 


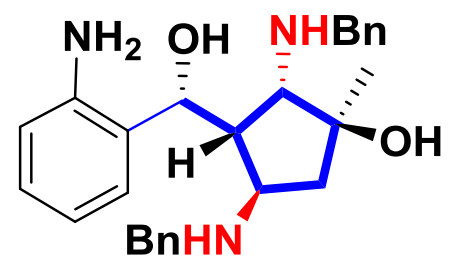

2,4-bis(benzylamino)-3-(hydroxy(2-nitrophenyl)methyl)-1-methylcyclopentan-1-ol (2e): BIX 1e reacted under reductive cleavage conditions (general method) and after extraction with pentanes, CB 2e was obtained as a yellow solid (16 mg, 98\%). MP: 87-88 ${ }^{\circ}$ C. TLC: $R_{f}: 0.03$ (1:1 heptanes/EtOAc). IR (thin film) 3382, 3319, 3035, 2914, 1511, 1455, 1383, 1262, $1067 \mathrm{~cm}^{-1}$. ${ }^{1}$ H-NMR $\left(400 \mathrm{MHz}, \mathrm{CDCl}_{3}\right): \delta$ 7.40-7.12 (m 9H), $7.07(\mathrm{t}, J=7.9 \mathrm{~Hz}, 1 \mathrm{H}), 6.97-6.93(\mathrm{~m}, 2 \mathrm{H})$, 6.68-6.50 (m, 2H), $4.59(\mathrm{~d}, J=10.3 \mathrm{~Hz}, 1 \mathrm{H}), 4.08(\mathrm{~d}, J=12.5 \mathrm{~Hz}, 1 \mathrm{H}), 3.85(\mathrm{~d}, J=12.6 \mathrm{~Hz}, 1 \mathrm{H})$, $3.42(\mathrm{~d}, J=8.6 \mathrm{~Hz}, 1 \mathrm{H}), 3.32-3.23(\mathrm{~m}, 2 \mathrm{H}), 3.06(\mathrm{t}, J=10.7 \mathrm{~Hz}, 1 \mathrm{H}), 2.81(\mathrm{~s}, 1 \mathrm{H}), 1.85$ (dd, $J=$ 13.8, $6.4 \mathrm{~Hz}, 1 \mathrm{H}), 1.66(\mathrm{~d}, J=13.6 \mathrm{~Hz}, 1 \mathrm{H}), 1.43(\mathrm{~s}, 3 \mathrm{H}) .{ }^{13} \mathbf{C}-\mathbf{N M R}\left(100 \mathrm{MHz}, \mathrm{CDCl}_{3}\right): \delta 146.1$, 139.3, 139.0, 129.1 (2C), 128.7 (2C), 128.3 (2C), 128.0 (2C), 127.5 (2C), 127.0 (2C), 124.6, 117.5, 116.7, 82.0, 76.3, 69.5, 58.6, 54.0, 51.2, 49.1, 43.2, 22.7 ppm. ESI-MS $\mathrm{m} / z$ (rel int): (pos) 432.2 $\left([\mathrm{M}+\mathrm{H}]^{+}, 100\right)$; (neg) $430.2\left([\mathrm{M}-\mathrm{H}]^{-}, 100\right)$. HRMS (ESI): $[\mathrm{M}+\mathrm{H}]^{+}$Calculated for: $\mathrm{C}_{27} \mathrm{H}_{34} \mathrm{~N}_{3} \mathrm{O}_{2}{ }^{+}$: 432.2646, found: 432.2638. Absolute difference (ppm): 1.85 .<smiles>COc1ccc([C@@H](O)[C@H]2[C@@H](N)C[C@](C)(O)[C@H]2Br)cc1</smiles>

2,4-bis(benzylamino)-3-(hydroxy(4-methoxyphenyl)methyl)-1-methylcyclopentan-1-ol (2f): BIX 1f reacted under reductive cleavage conditions (general method) and after extraction with pentanes, CB $2 f$ was obtained as a white solid (11 mg, 78\%). MP: $113-116^{\circ} \mathrm{C}$. TLC: $R_{f}: 0.02(1: 1$ heptanes/EtOAc). IR (thin film) 3305, 3284, 3023, 2931, 1612, 1515, 1367, 1265, $1250 \mathrm{~cm}^{-1}$. ${ }^{1} \mathbf{H}-N M R\left(400 \mathrm{MHz}, \mathrm{CDCl}_{3}\right): \delta 7.40(\mathrm{~d}, J=7.2 \mathrm{~Hz}, 2 \mathrm{H}), 7.35-7.21(\mathrm{~m} \mathrm{8H}), 7.10(\mathrm{~d}, J=7.1 \mathrm{~Hz}$, $2 \mathrm{H}), 6.87(\mathrm{~d}, J=8.6 \mathrm{~Hz}, 2 \mathrm{H}), 4.38(\mathrm{~d}, J=8.2 \mathrm{~Hz}, 1 \mathrm{H}), 4.22(\mathrm{~d}, J=14.6 \mathrm{~Hz}, 1 \mathrm{H}), 3.99(\mathrm{~d}, J=14.6$ $\mathrm{Hz}, 1 \mathrm{H}), 3.90(\mathrm{~s}, 3 \mathrm{H}), 3.71(\mathrm{~d}, J=13.0 \mathrm{~Hz}, 1 \mathrm{H}), 3.58(\mathrm{~d}, J=13.0 \mathrm{~Hz}, 1 \mathrm{H}), 3.47$ (dd, $J=8.5,1.5$ $\mathrm{Hz}, 1 \mathrm{H}), 3.23(\mathrm{~d}, J=4.7 \mathrm{~Hz}, 1 \mathrm{H}), 2.91(\mathrm{td}, J=8.4,1.6 \mathrm{~Hz}, 1 \mathrm{H}), 2.26(\mathrm{dd}, J=13.9,4.9 \mathrm{~Hz}, 1 \mathrm{H})$, $1.85(\mathrm{~d}, J=13.8,2.0 \mathrm{~Hz}, 1 \mathrm{H}), 1.42(\mathrm{~s}, 3 \mathrm{H}) .{ }^{13} \mathbf{C}-\mathbf{N M R}\left(100 \mathrm{MHz}, \mathrm{CDCl}_{3}\right): \delta 159.4,138.7,137.8$, 130.7, 129.0 (2C), 128.5 (2C), 128.3 (4C), 128.2 (2C), 127.3, 127.1, 114.0 (2C), 83.0, 81.2, 80.9, 63.7, 62.6, 60.5, 55.3, 51.3, 41.4, 21.5 ppm. ESI-MS $\mathrm{m} / z$ (rel int): (pos) $447.3\left([\mathrm{M}+\mathrm{H}]^{+}, 100\right)$; (neg) $445.3\left([\mathrm{M}-\mathrm{H}]^{-}, 100\right)$. HRMS (ESI): $[\mathrm{M}+\mathrm{H}]^{+}$Calculated for: $\mathrm{C}_{28} \mathrm{H}_{35} \mathrm{~N}_{2} \mathrm{O}_{3}{ }^{+}: 447.2642$, found: 447.2637. Absolute difference (ppm): 1.12. 


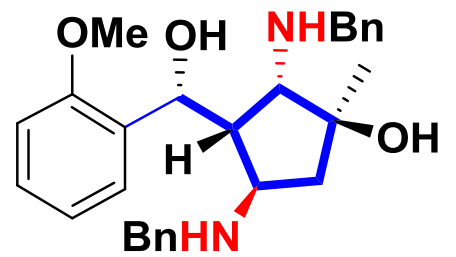

2,4-bis(benzylamino)-3-(hydroxy(2-methoxyphenyl)methyl)-1-methylcyclopentan-1-ol (2g): BIX 1 g reacted under reductive cleavage conditions (general method) and after extraction with pentanes, CB $2 \mathbf{g}$ was obtained as a white solid $(12 \mathrm{mg}, 88 \%)$. MP: $84-87^{\circ} \mathrm{C}$. TLC: $R_{f}: 0.03$ (1:1 heptanes/EtOAc). IR (thin film) 3245, 3216, 3054, 2944, 1601, 1493, 1454, 1264, 1250, $1160 \mathrm{~cm}^{-}$ ${ }^{1} .{ }^{1} \mathbf{H}-N M R\left(400 \mathrm{MHz}, \mathrm{CDCl}_{3}\right): \delta 7.53(\mathrm{~d}, J=7.7 \mathrm{~Hz}, 1 \mathrm{H}), 7.42-7.35(\mathrm{~m} 6 \mathrm{H}), 7.33-7.16(\mathrm{~m}, 3 \mathrm{H})$, 7.05-6.97 (m, 3H), $6.83(\mathrm{~d}, J=8.2 \mathrm{~Hz}, 1 \mathrm{H}), 5.19(\mathrm{~d}, J=7.4 \mathrm{~Hz}, 1 \mathrm{H}), 3.95(\mathrm{~d}, J=12.3 \mathrm{~Hz}, 1 \mathrm{H})$, $3.82(\mathrm{~d}, J=12.3 \mathrm{~Hz}, 1 \mathrm{H}), 3.73(\mathrm{~s}, 3 \mathrm{H}), 3.58-3.53(\mathrm{~m}, 2 \mathrm{H}), 3.23(\mathrm{~d}, J=7.7 \mathrm{~Hz}, 1 \mathrm{H}), 3.17(\mathrm{t}, J=$ $4.8 \mathrm{~Hz}, 1 \mathrm{H}), 2.75-2.26(\mathrm{~m}, 1 \mathrm{H}), 1.92(\mathrm{dd}, J=14.0,6.8 \mathrm{~Hz}, 1 \mathrm{H}), 1.64(\mathrm{~d}, J=13.8 \mathrm{~Hz}, 1 \mathrm{H}), 1.42(\mathrm{~s}$, 3H). ${ }^{13}$ C-NMR (100 MHz, CDCl 3 ): $\delta 156.1,139.5,139.4,131.9,128.8(2 \mathrm{C}), 128.5$ (2C), 128.3 (2C), $128.0(2 \mathrm{C}), 127.5(2 \mathrm{C}), 127.4,127.0,120.9,110.4,82.4,70.3,68.9,58.0,55.2,54.6,54.2$, 51.5, 43.6, 22.0 ppm. ESI-MS $\mathrm{m} / \mathrm{z}$ (rel int): (pos) 447.3 ([M+H] $\left.]^{+}, 100\right)$; (neg) $445.3\left([\mathrm{M}-\mathrm{H}]^{-}\right.$, 100). HRMS (ESI): $[\mathrm{M}+\mathrm{H}]^{+}$Calculated for: $\mathrm{C}_{28} \mathrm{H}_{35} \mathrm{~N}_{2} \mathrm{O}_{3}{ }^{+}: 447.2642$, found: 447.2649. Absolute difference (ppm): 1.57 .

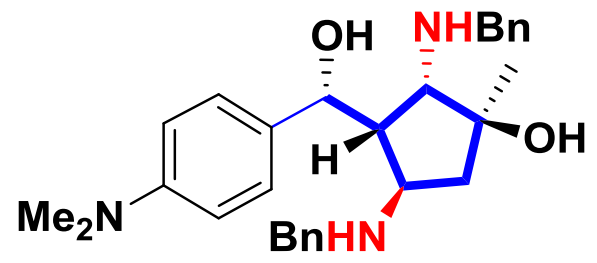

2,4-bis(benzylamino)-3-((4-(dimethylamino)phenyl)(hydroxy)methyl)-1-methylcyclopentan1-ol (2h): BIX $1 \mathrm{~h}$ reacted under reductive cleavage conditions (general method) and after extraction with pentanes, CB $\mathbf{2 h}$ was obtained as a yellow oil $(8 \mathrm{mg}, 87 \%)$. TLC: $R_{f}: 0.01$ (1:1 heptanes/EtOAc). IR (thin film) 3320, 3283, 3016, 2928, 1615, 1525, 1454, 1351, 1265, $1166 \mathrm{~cm}^{-}$ ${ }^{1}{ }^{1} \mathbf{H}-\mathbf{N M R}\left(400 \mathrm{MHz}, \mathrm{CDCl}_{3}\right): \delta 7.40(\mathrm{~d}, J=7.5 \mathrm{~Hz}, 2 \mathrm{H}), 7.36-7.18(\mathrm{~m}, 8 \mathrm{H}), 7.16-7.06(\mathrm{~m}, 2 \mathrm{H})$, $6.70(\mathrm{~d}, J=7.5 \mathrm{~Hz}, 2 \mathrm{H}), 4.35(\mathrm{~d}, J=8.3 \mathrm{~Hz}, 1 \mathrm{H}), 4.20(\mathrm{~d}, J=14.6 \mathrm{~Hz}, 1 \mathrm{H}), 3.98(\mathrm{~d}, J=14.6 \mathrm{~Hz}$, $1 \mathrm{H}), 3.72(\mathrm{~d}, J=12.9 \mathrm{~Hz}, 1 \mathrm{H}), 3.57(\mathrm{~d}, J=12.9 \mathrm{~Hz}, 1 \mathrm{H}), 3.46(\mathrm{~d}, J=8.5 \mathrm{~Hz}, 1 \mathrm{H}), 3.21(\mathrm{~d}, J=4.6$ $\mathrm{Hz}, 1 \mathrm{H}), 2.97-2.92(\mathrm{~m}, 7 \mathrm{H}), 2.25(\mathrm{dd}, J=13.9,4.7 \mathrm{~Hz}, 1 \mathrm{H}), 1.87(\mathrm{~d}, J=14.0 \mathrm{~Hz}, 1 \mathrm{H}), 1.41(\mathrm{~s}$, $3 \mathrm{H}) .{ }^{13} \mathbf{C}-\mathbf{N M R}\left(100 \mathrm{MHz}, \mathrm{CDCl}_{3}\right): \delta 150.7,138.6,137.9,129.3,129.1,128.7,128.6,128.5(2 \mathrm{C})$, $128.3,128.1,127.5,127.3,127.1,127.0,125.8,112.6,112.4,83.4,81.2,80.9,63.3,62.6,60.6$, 51.3, 41.2, 40.5 (2C), 21.5 ppm. ESI-MS $m / z$ (rel int): (pos) 460.3 ([M+H $]^{+}, 100$ ); (neg) 458.3 ([M-H] $\left.]^{-}, 100\right)$. HRMS (ESI): $[\mathrm{M}+\mathrm{H}]^{+}$Calculated for: $\mathrm{C}_{29} \mathrm{H}_{38} \mathrm{~N}_{3} \mathrm{O}_{2}{ }^{+}: 460.2959$, found: 460.2964 . Absolute difference (ppm): 1.09 . 


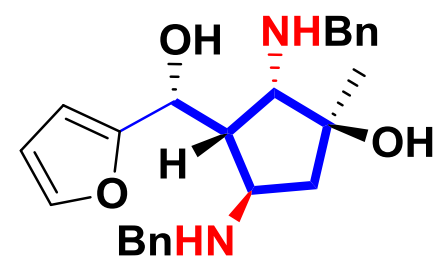

2,4-bis(benzylamino)-3-((4-(dimethylamino)phenyl)(hydroxy)methyl)-1-methylcyclopentan1-ol (2i): BIX 1i reacted under reductive cleavage conditions (general method) and after extraction with pentanes, CB $2 \mathbf{i}$ was obtained as a light yellow solid $(10 \mathrm{mg}, 87 \%)$. MP: $106-109^{\circ} \mathrm{C}$. TLC: $R_{f}: 0.15$ (1:1 heptanes/EtOAc). IR (thin film) 3267, 3221, 3032, 2930, 1604, 1496, 1453, 1363, $1266 \mathrm{~cm}^{-1} .{ }^{1} \mathbf{H}-\mathbf{N M R}\left(400 \mathrm{MHz}, \mathrm{CDCl}_{3}\right): \delta 7.38(\mathrm{~s}, 1 \mathrm{H}), 7.35-7.20(\mathrm{~m}, 8 \mathrm{H}), 7.16(\mathrm{~d}, J=7.2 \mathrm{~Hz}$, $2 \mathrm{H}), 6.36(\mathrm{~s}, 1 \mathrm{H}), 6.29(\mathrm{~s}, 1 \mathrm{H}), 4.81(\mathrm{~d}, J=7.1 \mathrm{~Hz}, 1 \mathrm{H}), 3.86(\mathrm{~d}, J=12.1 \mathrm{~Hz}, 1 \mathrm{H}), 3.75-3.62(\mathrm{~m}$, 2H), $3.57(\mathrm{~d}, J=13.2 \mathrm{~Hz}, 1 \mathrm{H}), 3.35$ (d, $J=7.5 \mathrm{~Hz}, 1 \mathrm{H}), 3.21$ (t, $J=4.6 \mathrm{~Hz}, 1 \mathrm{H}), 2.76-2.72(\mathrm{~m}$, 1H), $1.83(\mathrm{dd}, J=14.3,6.4 \mathrm{~Hz}, 1 \mathrm{H}), 1.70(\mathrm{~d}, J=13.9 \mathrm{~Hz}, 1 \mathrm{H}), 1.42(\mathrm{~s}, 3 \mathrm{H}) .{ }^{13} \mathrm{C}-\mathbf{N M R}(100 \mathrm{MHz}$, $\left.\mathrm{CDCl}_{3}\right): \delta 156.5,141.8,139.2,139.1,128.8(2 \mathrm{C}), 128.5(2 \mathrm{C}), 128.4(2 \mathrm{C}), 128.0(2 \mathrm{C}), 127.6,127.2$, 110.3, 106.7, 82.6, 70.5, 69.0, 58.7, 54.7, 52.5, 51.7, 42.8, 21.9 ppm. ESI-MS $m / z$ (rel int): (pos) $407.2\left([\mathrm{M}+\mathrm{H}]^{+}, 100\right)$; (neg) $405.2\left([\mathrm{M}-\mathrm{H}]^{-}, 100\right)$. HRMS (ESI): $[\mathrm{M}+\mathrm{H}]^{+}$Calculated for: $\mathrm{C}_{25} \mathrm{H}_{31} \mathrm{~N}_{2} \mathrm{O}_{3}{ }^{+}$: 407.2329, found: 407.2321. Absolute difference (ppm): 1.96 .

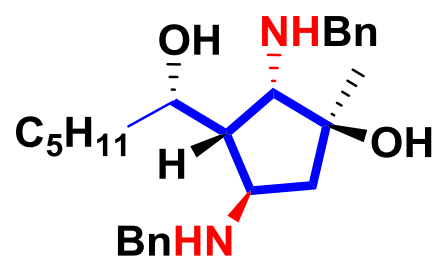

2,4-bis(benzylamino)-3-(1-hydroxyhexyl)-1-methylcyclopentan-1-ol (2j): BIX $\mathbf{1 j}$ reacted under reductive cleavage conditions (general method) and after extraction with pentanes, CB $\mathbf{2} \mathbf{j}$ was obtained as a light yellow oil $(16 \mathrm{mg}, 95 \%)$. TLC: $R_{f}: 0.02$ (1:1 heptanes/EtOAc). IR (thin film) 3345, 3311, 3053, 2931, 1511, 1453, 1358, 1264, 1133, $1098 \mathrm{~cm}^{-1} .{ }^{\mathbf{1}} \mathbf{H}-\mathbf{N M R}(400 \mathrm{MHz}$, $\left.\mathrm{CDCl}_{3}\right): \delta 7.35-7.25(\mathrm{~m}, 10 \mathrm{H}), 3.97(\mathrm{~d}, J=12.2 \mathrm{~Hz}, 1 \mathrm{H}), 3.89-3.78(\mathrm{~m}, 2 \mathrm{H}), 3.73-3.58(\mathrm{~m}, 2 \mathrm{H})$, $3.28(\mathrm{~d}, J=7.3 \mathrm{~Hz}, 1 \mathrm{H}), 3.21(\mathrm{t}, J=5.4 \mathrm{~Hz}, 1 \mathrm{H}), 2.15(\mathrm{t}, J=5.8 \mathrm{~Hz}, 1 \mathrm{H}), 1.83(\mathrm{dd}, J=14.1,6.9$ $\mathrm{Hz}, 1 \mathrm{H}), 1.72(\mathrm{~d}, J=13.9 \mathrm{~Hz}, 1 \mathrm{H}), 1.59-1.45(\mathrm{~m}, 2 \mathrm{H}), 1.44(\mathrm{~s}, 3 \mathrm{H}), 1.38-1.21(\mathrm{~m}, 6 \mathrm{H}), 0.89$ (t, $J$ $=6.9 \mathrm{~Hz}, 3 \mathrm{H}) .{ }^{13} \mathbf{C}-\mathbf{N M R}\left(100 \mathrm{MHz}, \mathrm{CDCl}_{3}\right): \delta 139.1,128.9,128.8,128.7,128.6(2 \mathrm{C}), 128.5$, 128.3 (2C), 127.6, 127.4 (2C), 82.6, 72.5, 70.2, 58.7, 54.7, 53.7, 51.9, 42.7, 36.8, 32.0, 25.8, 22.6, 22.2, 14.1 ppm. ESI-MS m/z (rel int): (pos) 411.3 ([M+H] $\left.]^{+}, 100\right)$; (neg) 409.3 ([M-H $\left.]^{-}, 100\right)$. HRMS (ESI): $[\mathrm{M}+\mathrm{H}]^{+}$Calculated for: $\mathrm{C}_{26} \mathrm{H}_{39} \mathrm{~N}_{2} \mathrm{O}_{2}{ }^{+}$: 411.3006, found: 411.3012. Absolute difference (ppm): 1.46 . 


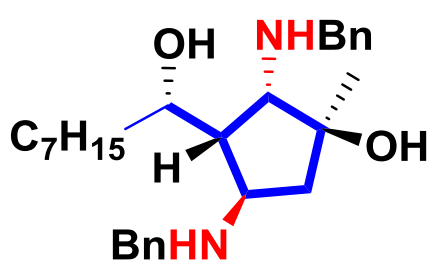

2,4-bis(benzylamino)-3-(1-hydroxyoctyl)-1-methylcyclopentan-1-ol (2k): BIX 1k reacted under reductive cleavage conditions (general method) and after extraction with pentanes, CB $\mathbf{2 k}$ was obtained as a light yellow oil $(8 \mathrm{mg}, 76 \%)$. TLC: $R_{f}: 0.01(1: 1$ heptanes/EtOAc). IR (thin film) 3321, 3280, 3035, 2928, 1601, 1520, 1453, 1358, 1265, 1106, $1070 \mathrm{~cm}^{-1}$. ${ }^{1} \mathbf{H}-\mathbf{N M R}$ $\left(400 \mathrm{MHz}, \mathrm{CDCl}_{3}\right): \delta 7.51-7.14(\mathrm{~m}, 10 \mathrm{H}), 4.11(\mathrm{~d}, J=14.6 \mathrm{~Hz}, 1 \mathrm{H}), 3.89-3.81(\mathrm{~m}, 2 \mathrm{H}), 3.71(\mathrm{~d}$, $J=13.0 \mathrm{~Hz}, 1 \mathrm{H}), 3.49-3.42(\mathrm{~m}, 1 \mathrm{H}), 3.28(\mathrm{dd}, J=8.3,1.5 \mathrm{~Hz}, 1 \mathrm{H}), 3.09(\mathrm{~d}, J=4.7 \mathrm{~Hz}, 1 \mathrm{H}), 2.55$ $(\mathrm{dd}, J=8.2,1.5 \mathrm{~Hz}, 1 \mathrm{H}), 2.12(\mathrm{dd}, J=13.8,4.8 \mathrm{~Hz}, 1 \mathrm{H}), 1.80(\mathrm{~d}, J=13.8 \mathrm{~Hz}, 1 \mathrm{H}), 1.73(\mathrm{~d}, J=$ $2.8 \mathrm{~Hz}, 1 \mathrm{H}), 1.65-1.58(\mathrm{~m}, 1 \mathrm{H}), 1.55-1.48(\mathrm{~m}, 1 \mathrm{H}), 1.36(\mathrm{~s}, 3 \mathrm{H}), 1.32-1.21(\mathrm{~m}, 9 \mathrm{H}), 0.88(\mathrm{t}, J=$ $6.9 \mathrm{~Hz}, 3 \mathrm{H}) .{ }^{13} \mathrm{C}-\mathrm{NMR}\left(100 \mathrm{MHz}, \mathrm{CDCl}_{3}\right): \delta 138.2,129.5,129.0,128.9,128.7(2 \mathrm{C}), 128.3(2 \mathrm{C})$, $128.2,128.1,127.5,127.0,81.2,80.9,80.7,62.7,61.5,61.1,60.4,51.5,41.1,33.4,31.9,29.7$, 26.2, 22.7, 21.5, 14.1 ppm. ESI-MS m/z (rel int): (pos) $439.3\left([\mathrm{M}+\mathrm{H}]^{+}, 100\right)$; (neg) $437.3\left([\mathrm{M}-\mathrm{H}]^{-}\right.$ , 100). HRMS (ESI): $[\mathrm{M}+\mathrm{H}]^{+}$Calculated for: $\mathrm{C}_{28} \mathrm{H}_{43} \mathrm{~N}_{2} \mathrm{O}_{2}{ }^{+}: 439.3319$, found: 439.3324 . Absolute difference (ppm): 1.14 .

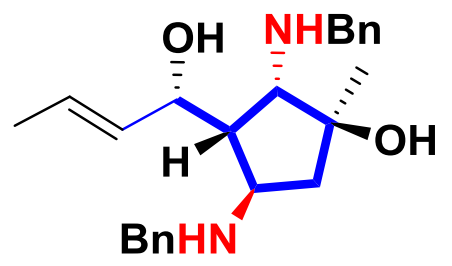

2,4-bis(benzylamino)-3-((E)-1-hydroxybut-2-en-1-yl)-1-methylcyclopentan-1-ol (2I): BIX 11 reacted under reductive cleavage conditions (general method) and after extraction with pentanes, CB 2l was obtained as a light yellow oil (14 mg, 86\%). TLC: $R_{f}: 0.01$ (1:1 heptanes/EtOAc). IR (thin film) 3364, 3324, 3054, 2935, 1601, 1554, 1453, 1304, 1264, 1108, $1061 \mathrm{~cm}^{-1}$. ${ }^{\mathbf{1}} \mathbf{H}-\mathbf{N M R}$ $\left(400 \mathrm{MHz}, \mathrm{CDCl}_{3}\right): \delta 7.45-7.19(\mathrm{~m}, 10 \mathrm{H}), 5.70(\mathrm{dq}, J=14.8,7.1 \mathrm{~Hz}, 1 \mathrm{H}), 5.49(\mathrm{dd}, J=15.4,6.8$ $\mathrm{Hz}, 1 \mathrm{H}), 4.10(\mathrm{t}, J=7.3 \mathrm{~Hz}, 1 \mathrm{H}), 3.97(\mathrm{~d}, J=12.3 \mathrm{~Hz}, 1 \mathrm{H}), 3.88-3.73(\mathrm{~m}, 2 \mathrm{H}), 3.64(\mathrm{~d}, J=13.0$ $\mathrm{Hz}, 1 \mathrm{H}), 3.33(\mathrm{~d}, J=7.7 \mathrm{~Hz}, 1 \mathrm{H}), 3.13(\mathrm{~s}, 1 \mathrm{H}), 2.27-2.14(\mathrm{~m}, 1 \mathrm{H}), 1.82(\mathrm{dd}, J=14.0,6.8 \mathrm{~Hz}, 1 \mathrm{H})$, 1.74-1.165 (m, 4H), 1.42 (s, 3H). ${ }^{13} \mathbf{C}-\mathbf{N M R}\left(100 \mathrm{MHz}, \mathrm{CDCl}_{3}\right): \delta 1393,139.2,133.0,128.7(2 \mathrm{C})$, 128.6 (2C), 128.5 (2C), 128.4, 128.2 (2C), 127.6, 127.3, 82.5, 73.4, 70.1, 58.5, 54.5, 54.0, 51.9, 42.9, 22.2, 17.8 ppm. ESI-MS m/z (rel int): (pos) $381.3\left([\mathrm{M}+\mathrm{H}]^{+}, 100\right)$; (neg) $379.3\left([\mathrm{M}-\mathrm{H}]^{-}\right.$, 100). HRMS (ESI): $[\mathrm{M}+\mathrm{H}]^{+}$Calculated for: $\mathrm{C}_{24} \mathrm{H}_{33} \mathrm{~N}_{2} \mathrm{O}_{2}{ }^{+}: 381.2537$, found: 381.2543 . Absolute difference (ppm): 1.57 . 


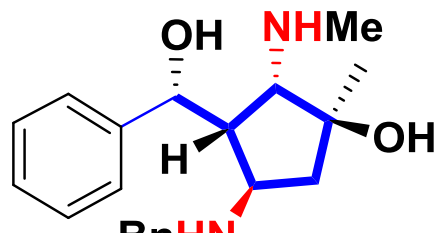

BnHN

4-(benzylamino)-3-(hydroxy(phenyl)methyl)-1-methyl-2-(methylamino) cyclopentan-1-ol (2m): BIX $1 \mathrm{~m}$ reacted under reductive cleavage conditions (general method) and after extraction with pentanes, CB $\mathbf{2 m}$ was obtained as a colorless oil (12 mg, 75\%). TLC: $R_{f}: 0.13$ (1:1 heptanes/EtOAc). IR (thin film) 3320, 3247, 3054, 2935, 1508, 1453, 1366, 1264, 1154, $1098 \mathrm{~cm}^{-}$ ${ }^{1} .{ }^{1} \mathbf{H}-\mathbf{N M R}\left(400 \mathrm{MHz}, \mathrm{CDCl}_{3}\right): \delta 7.48-7.15(\mathrm{~m}, 8 \mathrm{H}), 7.01-6.97(\mathrm{~m}, 2 \mathrm{H}), 4.68(\mathrm{~d}, J=8.1 \mathrm{~Hz}, 1 \mathrm{H})$, $3.54(\mathrm{~d}, J=13.1 \mathrm{~Hz}, 1 \mathrm{H}), 3.40(\mathrm{~d}, J=13.1 \mathrm{~Hz}, 1 \mathrm{H}), 3.07(\mathrm{~d}, J=8.6 \mathrm{~Hz}, 2 \mathrm{H}), 2.53-2.49(\mathrm{~m}, 4 \mathrm{H})$, $1.86(\mathrm{~d}, J=14.1 \mathrm{~Hz}, 1 \mathrm{H}), 1.70(\mathrm{~d}, J=14.1 \mathrm{~Hz}, 1 \mathrm{H}), 1.38(\mathrm{~s}, 3 \mathrm{H}) .{ }^{13} \mathbf{C}-\mathbf{N M R}\left(100 \mathrm{MHz}, \mathrm{CDCl}_{3}\right)$ : $\delta$ 143.4, 138.8, 128.7 (2C), 128.6, 128.5 (2C), 128.2, 128.0, 127.5, 127.2, 126.6, 82.0, 74.8, 72.3, 58.1, 55.1, 51.6, 43.0, 36.8, $22.2 \mathrm{ppm}$. ESI-MS $\mathrm{m} / \mathrm{z}$ (rel int): (pos) $341.2\left([\mathrm{M}+\mathrm{H}]^{+}, 100\right)$; (neg) $339.2\left([\mathrm{M}-\mathrm{H}]^{-}, 100\right)$. HRMS (ESI): $[\mathrm{M}+\mathrm{H}]^{+}$Calculated for: $\mathrm{C}_{21} \mathrm{H}_{29} \mathrm{~N}_{2} \mathrm{O}_{2}{ }^{+}$: 341.2224 , found: 341.2228. Absolute difference (ppm): 1.17.

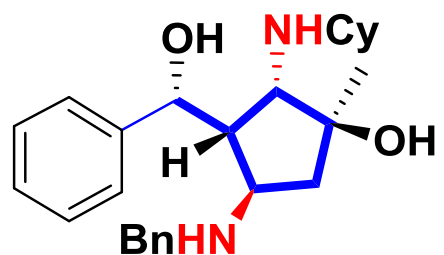

4-(benzylamino)-2-(cyclohexylamino)-3-(hydroxy(phenyl)methyl)-1-methylcyclopentan-1-ol (2n): BIX 1n reacted under reductive cleavage conditions (general method) and after extraction with pentanes, CB 2n was obtained as a light yellow oil (16 mg, 91\%). TLC: $R_{f}: 0.10$ (1:1 heptanes/EtOAc). IR (thin film) 3388, 3328, 3050, 2928, 1513, 1452, 1340, 1265, 1133, $1098 \mathrm{~cm}^{-}$ 1. ${ }^{1}$ H-NMR $\left(400 \mathrm{MHz}, \mathrm{CDCl}_{3}\right): \delta 7.50-7.11(\mathrm{~m}, 8 \mathrm{H}), 6.93-6.89(\mathrm{~m}, 2 \mathrm{H}), 4.58(\mathrm{~d}, J=9.2 \mathrm{~Hz}, 1 \mathrm{H})$, 3.48-3.42 (m, 2H), $3.31(\mathrm{~d}, J=13.2 \mathrm{~Hz}, 1 \mathrm{H}), 2.92$ (t, $J=3.8 \mathrm{~Hz}, 1 \mathrm{H}), 2.53-2.49$ (m. 2H), 1.99 (d, $J=12.7 \mathrm{~Hz}, 1 \mathrm{H}), 1.88-1.82(\mathrm{~m}, 2 \mathrm{H}), 1.72-1.67(\mathrm{~m}, 3 \mathrm{H}), 1.63-1.58(\mathrm{~m}, 1 \mathrm{H}), 1.36(\mathrm{~s}, 3 \mathrm{H}), 1.33-$ 1.21(m, 4H), 1.18-1.14 (m, 3H), 1.03-0.98 (m, 1H). ${ }^{13}$ C-NMR $\left(100 \mathrm{MHz}, \mathrm{CDCl}_{3}\right): \delta 142.9,138.8$, 128.4 (4C), 128.0 (2C), 127.8, 127.6, 127.1, 127.0, 81.9, 74.8, 66.9, 58.0, 56.8, 54.9, 51.4, 43.1, 34.0, 33.2, 25.8, 25.2, 24.7, $22.6 \mathrm{ppm}$. ESI-MS $\mathrm{m} / \mathrm{z}$ (rel int): (pos) 409.3 ([M+H] $\left.]^{+}, 100\right)$; (neg) $407.3\left([\mathrm{M}-\mathrm{H}]^{-}, 100\right)$. HRMS (ESI): $[\mathrm{M}+\mathrm{H}]^{+}$Calculated for: $\mathrm{C}_{26} \mathrm{H}_{37} \mathrm{~N}_{2} \mathrm{O}_{2}{ }^{+}:$409.2850, found: 409.2855. Absolute difference (ppm): 1.22. 


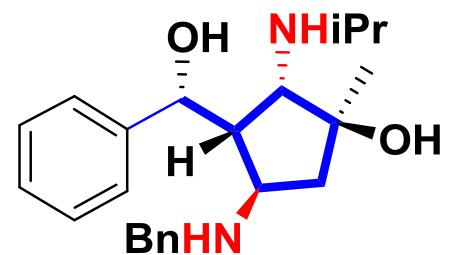

4-(benzylamino)-3-(hydroxy(phenyl)methyl)-2-(isopropylamino)-1-methylcyclopentan-1-ol (2o): BIX 10 reacted under reductive cleavage conditions (general method) and after extraction with pentanes, CB 20 was obtained as a light yellow oil (15 mg, 76\%). TLC: $R_{f}: 0.05$ (1:1 heptanes/EtOAc). IR (thin film) 3405, 3320, 3035, 2965, 1516, 1454, 1385, 1265, 1136, $1067 \mathrm{~cm}^{-}$ ${ }^{1} .{ }^{1} \mathbf{H}-N M R\left(400 \mathrm{MHz}, \mathrm{CDCl}_{3}\right): \delta 7.53-7.20(\mathrm{~m}, 8 \mathrm{H}), 7.10-7.05(\mathrm{~m}, 2 \mathrm{H}), 4.92(\mathrm{~d}, J=8.2 \mathrm{~Hz}, 1 \mathrm{H})$, $3.62-3.51(\mathrm{~m}, 2 \mathrm{H}), 3.43(\mathrm{~d}, J=13.1 \mathrm{~Hz}, 1 \mathrm{H}), 3.26(\mathrm{~d}, J=6.6 \mathrm{~Hz}, 1 \mathrm{H}), 3.15-3.02(\mathrm{~m}, 1 \mathrm{H}), 2.93-$ $2.86(\mathrm{~m}, 1 \mathrm{H}), 2.21(\mathrm{dd}, J=14.1,7.7 \mathrm{~Hz}, 1 \mathrm{H}), 2.01(\mathrm{dd}, J=14.2,4.4 \mathrm{~Hz}, 1 \mathrm{H}), 1.46(\mathrm{~s}, 3 \mathrm{H}), 1.24$ $(\mathrm{d}, J=6.2 \mathrm{~Hz}, 3 \mathrm{H}), 1.10(\mathrm{~d}, J=6.2 \mathrm{~Hz}, 3 \mathrm{H}) .{ }^{13} \mathbf{C}-\mathbf{N M R}\left(100 \mathrm{MHz}, \mathrm{CDCl}_{3}\right): \delta 142.1,128.8(2 \mathrm{C})$, 128.7 (2C), 128.6 (2C), 128.0 (2C), 127.9 (2C), 126.8, 80.2, 73.2, 67.3, 57.2, 51.8, 51.0, 50.3, 42.4, 23.4, 22.0, 21.4 ppm. ESI-MS m/z (rel int): (pos) 369.3 ([M+H $\left.]^{+}, 100\right)$; (neg) 367.3 ([M-H] $]^{-}$ , 100). HRMS (ESI): [M+H] $]^{+}$Calculated for: $\mathrm{C}_{23} \mathrm{H}_{33} \mathrm{~N}_{2} \mathrm{O}_{2}{ }^{+}: 369.2537$, found: 369.2543 . Absolute difference (ppm): 1.63 .

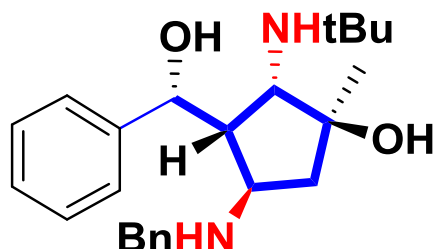

4-(benzylamino)-3-(hydroxy(phenyl)methyl)-2-(isopropylamino)-1-methylcyclopentan-1-ol (2p): BIX 1p reacted under reductive cleavage conditions (general method) and after extraction with pentanes, CB 2p was obtained as a colorless oil $(18 \mathrm{mg}, 93 \%)$. TLC: $R_{f}$ : 0.03 (1:1 heptanes/EtOAc). IR (thin film) 3425, 3340, 3023, 2965, 1511, 1444, 1265, 1126, $1065 \mathrm{~cm}^{-1}$. ${ }^{1}$ H-NMR (400 MHz, $\left.\mathrm{CDCl}_{3}\right): \delta$ 7.41-7.12 (m, 8H), 6.91-6.84 (m, 2H), $4.58(\mathrm{~d}, J=10.3 \mathrm{~Hz}, 1 \mathrm{H})$, $3.66(\mathrm{~d}, J=9.1 \mathrm{~Hz}, 1 \mathrm{H}), 3.40(\mathrm{~d}, J=12.8 \mathrm{~Hz}, 1 \mathrm{H}), 3.21(\mathrm{~d}, J=12.8 \mathrm{~Hz}, 1 \mathrm{H}), 2.62-2.58(\mathrm{~m}, 1 \mathrm{H})$, $2.14(\mathrm{t}, J=9.7 \mathrm{~Hz}, 1 \mathrm{H}), 2.03(\mathrm{dd}, J=13.0,5.7 \mathrm{~Hz}, 1 \mathrm{H}), 1.71(\mathrm{dd}, J=13.5,5.6 \mathrm{~Hz}, 1 \mathrm{H}), 1.34(\mathrm{~s}$, 3H), $1.22(\mathrm{~s}, 9 \mathrm{H}) .{ }^{13} \mathbf{C}-\mathbf{N M R}\left(100 \mathrm{MHz}, \mathrm{CDCl}_{3}\right): \delta 142.5,139.3,128.5,128.4,128.3,128.0,127.8$ (2C), 127.2 (2C), 127.0, 126.9, 78.7, 76.8, 64.9, 56.8, 53.6, 51.8, 51.6, 45.8, 29.4 (3C), 23.9 ppm. ESI-MS $\mathrm{m} / \mathrm{z}$ (rel int): (pos) $383.3\left([\mathrm{M}+\mathrm{H}]^{+}, 100\right)$; (neg) $381.3\left([\mathrm{M}-\mathrm{H}]^{-}, 100\right)$. HRMS (ESI): $[\mathrm{M}+\mathrm{H}]^{+}$Calculated for: $\mathrm{C}_{24} \mathrm{H}_{35} \mathrm{~N}_{2} \mathrm{O}_{2}{ }^{+}: 383.2693$, found: 383.2687. Absolute difference (ppm): 1.57 . 


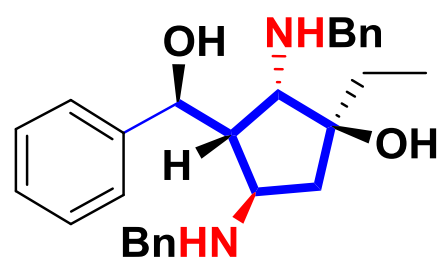

2,4-bis(benzylamino)-1-ethyl-3-(hydroxy(phenyl)methyl)cyclopentan-1-ol (2q): BIX 1q reacted under reductive cleavage conditions (general method) and after extraction with pentanes, CB 2q was obtained as a light yellow oil (11 mg, 75\%). TLC: $R_{f}: 0.03$ (1:1 heptanes/EtOAc). IR (thin film) $3416,3302,3032,2964,1514,1454,1385,1262,1132,1024 \mathrm{~cm}^{-1} .{ }^{1} \mathbf{H}-\mathbf{N M R}(400 \mathrm{MHz}$, $\left.\mathrm{CDCl}_{3}\right): \delta$ 7.49-7.18 (m, 13H), 7.12-67.02 (m, 2H), $5.57(\mathrm{bs}, 2 \mathrm{H}), 4.40(\mathrm{~d}, J=8.0 \mathrm{~Hz}, 1 \mathrm{H}), 4.22$ $(\mathrm{d}, J=14.7 \mathrm{~Hz}, 1 \mathrm{H}), 3.97(\mathrm{~d}, J=14.8 \mathrm{~Hz}, 1 \mathrm{H}), 3.72-3.62(\mathrm{~m}, 2 \mathrm{H}), 3.51(\mathrm{~d}, J=8.5 \mathrm{~Hz}, 1 \mathrm{H}), 3.29$ $(\mathrm{d}, J=4.8 \mathrm{~Hz}, 1 \mathrm{H}), 3.04(\mathrm{t}, J=8.4 \mathrm{~Hz}, 1 \mathrm{H}), 2.15(\mathrm{~d}, J=13.4 \mathrm{~Hz}, 1 \mathrm{H}), 2.06(\mathrm{~d}, J=13.4 \mathrm{~Hz}, 1 \mathrm{H})$, $1.83(\mathrm{dq}, J=8.0,7.5 \mathrm{~Hz}, 1 \mathrm{H}), 1.72(\mathrm{dq}, J=8.0,7.5 \mathrm{~Hz}, 1 \mathrm{H}), 1.08(\mathrm{t}, J=7.5 \mathrm{~Hz}, 3 \mathrm{H}) .{ }^{13} \mathbf{C}-\mathbf{N M R}$ $\left(100 \mathrm{MHz}, \mathrm{CDCl}_{3}\right): \delta 138.8,137.8,137.3(2 \mathrm{C}), 128.8(2 \mathrm{C}), 128.6(2 \mathrm{C}), 128.3(2 \mathrm{C}), 128.2(2 \mathrm{C})$, 127.6 (2C), 127.1 (2C), 126.9 (2C), 84.0, 83.4, 80.7, 63.0, 62.5, 60.0, 50.8, 38.0, 27.6, 8.9 ppm. ESI-MS $m / z$ (rel int): (pos) $431.3\left([\mathrm{M}+\mathrm{H}]^{+}, 100\right)$; (neg) $429.3\left([\mathrm{M}-\mathrm{H}]^{-}, 100\right)$. HRMS (ESI): $[\mathrm{M}+\mathrm{H}]^{+}$Calculated for: $\mathrm{C}_{28} \mathrm{H}_{35} \mathrm{~N}_{2} \mathrm{O}_{2}{ }^{+}: 431.2693$, found: 431.2698. Absolute difference (ppm): 1.16. 


\section{X-RAy CRYstallograPhy DATA}

Samples preparation:

BIX 1f: Crystallized from an EtOAc/heptane solution.

CB 2e: Crystallized from pentanes.

\begin{tabular}{|c|c|c|}
\hline Crystal data & BIX 1f & CB2 2e \\
\hline Chemical formula & $\mathrm{C}_{28} \mathrm{H}_{30} \mathrm{~N}_{2} \mathrm{O}_{3}$ & $\mathrm{C}_{27} \mathrm{H}_{33} \mathrm{~N}_{3} \mathrm{O}_{2}$ \\
\hline$M_{\mathrm{r}}$ & 442.54 & 431.56 \\
\hline Crystal system & Triclinic & Orthorhombic \\
\hline space group & $P \overline{1}$ & $P 2{ }_{1} 2_{1} 2$ \\
\hline Temperature (K) & 100 & 100 \\
\hline$a(\AA)$ & $9.4356(4)$ & $13.9949(2)$ \\
\hline$b(\AA)$ & $10.6363(5)$ & $23.7478(4)$ \\
\hline$c(\AA)$ & $12.5968(5)$ & $7.0510(1)$ \\
\hline$\alpha\left(^{\circ}\right)$ & $106.8305(11)$ & 90 \\
\hline$\beta\left(^{\circ}\right)$ & $100.5058(11)$ & 90 \\
\hline$\gamma\left({ }^{\circ}\right)$ & $100.4574(11)$ & 90 \\
\hline$V\left(\AA^{3}\right)$ & $1152.32(9)$ & $2343.39(6)$ \\
\hline$Z$ & 2 & 4 \\
\hline Radiation type & Mo $K \alpha$ & Mo $K \alpha$ \\
\hline$\mu\left(\mathrm{mm}^{-1}\right)$ & 0.08 & 0.61 \\
\hline Crystal Density $\left(\mathrm{gcm}^{-3}\right)$ & 1.275 & 1.223 \\
\hline Crystal size $(\mathrm{mm})$ & $0.44 \times 0.24 \times 0.20$ & $0.21 \times 0.13 \times 0.12$ \\
\hline Diffractometer & $\begin{array}{l}\text { Bruker Photon } 100 \\
\text { CMOS } \\
\text { diffractometer }\end{array}$ & $\begin{array}{l}\text { Bruker D8 Venture } \\
\text { Photon } 2 \text { CMOS } \\
\text { diffractometer }\end{array}$ \\
\hline Absorption correction & $\begin{array}{l}\text { Multi-scan } \\
\text { SADABS V2014/5 } \\
\text { (Bruker AXS Inc.) }\end{array}$ & $\begin{array}{l}\text { Multi-scan } \\
\text { SADABS V2014/5 } \\
\text { (Bruker AXS Inc.) }\end{array}$ \\
\hline$T_{\min }$ & 0.94 & 0.82 \\
\hline$T_{\max }$ & 0.98 & 0.93 \\
\hline $\begin{array}{l}\text { No. of reflections } \\
\text { measured }\end{array}$ & 33951 & 20202 \\
\hline $\begin{array}{l}\text { No. of observed } \\
\text { reflections }[I>2 \sigma(I)]\end{array}$ & 6973 & 4118 \\
\hline$R_{\text {int }}$ & 0.030 & 0.039 \\
\hline$(\sin \theta / \lambda)_{\max }\left(\AA^{-1}\right)$ & 0.769 & 0.603 \\
\hline$R\left[F^{2}>2 \sigma\left(F^{2}\right)\right]$ & 0.052 & 0.028 \\
\hline$w R\left(F^{2}\right)$ & 0.134 & 0.066 \\
\hline$S$ & 1.03 & 1.05 \\
\hline No. of reflections & 8789 & 4302 \\
\hline
\end{tabular}




\begin{tabular}{|l|l|l|}
\hline No. of parameters & 300 & 313 \\
\hline H-atom treatment & $\begin{array}{l}\text { H-atom parameters } \\
\text { constrained }\end{array}$ & $\begin{array}{l}\text { H atoms treated by a } \\
\text { mixture of independent } \\
\text { and constrained } \\
\text { refinement }\end{array}$ \\
\hline$\Delta \rho_{\max }, \Delta \rho_{\min }\left(\mathrm{e} \AA^{-3}\right)$ & $0.56,-0.34$ & $0.14,-0.15$ \\
\hline Ellipsoid contour $\%$ & 35 & 35 \\
\hline
\end{tabular}

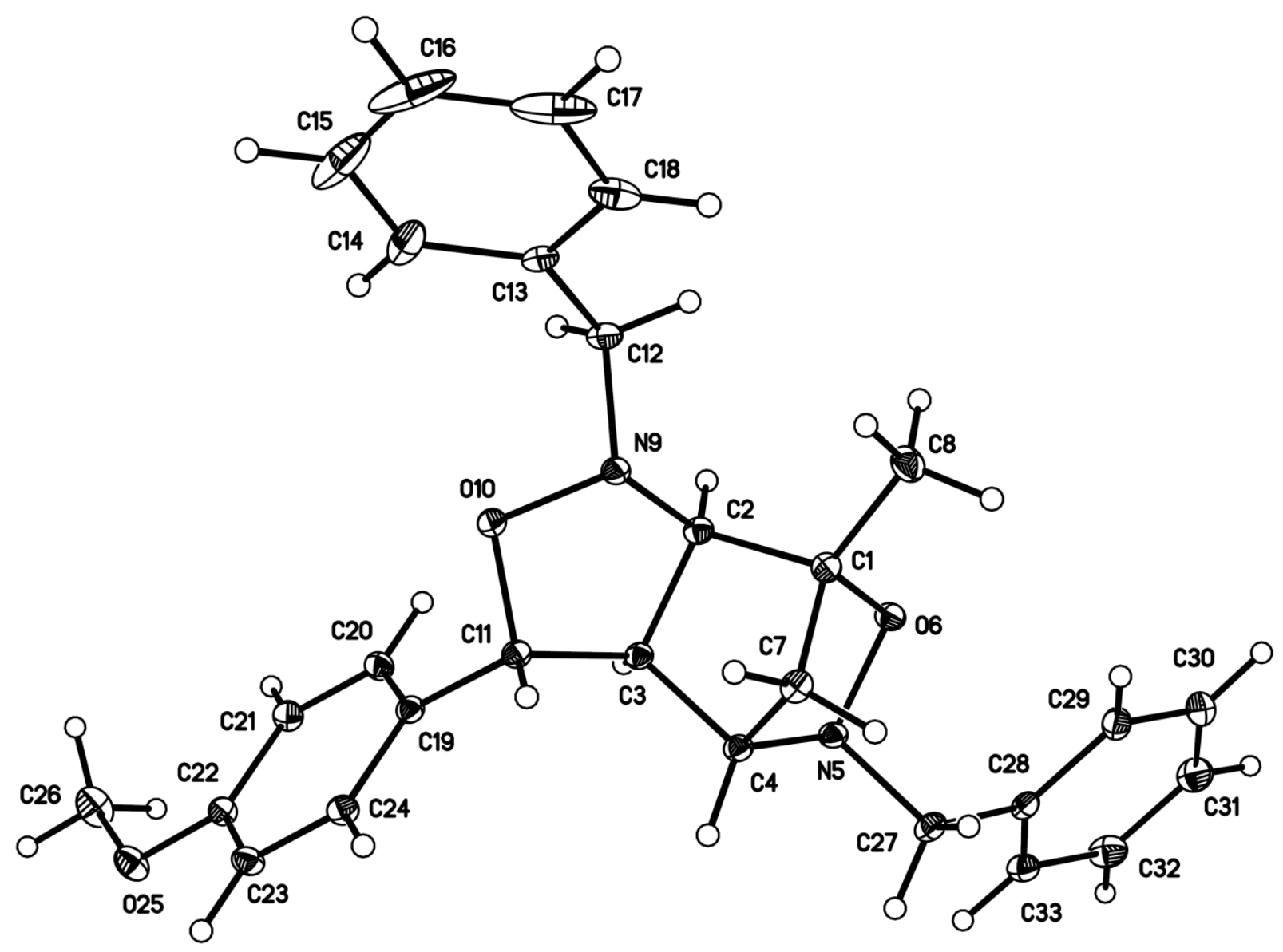

X-ray figure (CCDC 1937211, ellipsoid contour \% probability 35\%) of ent-BIX 1f 


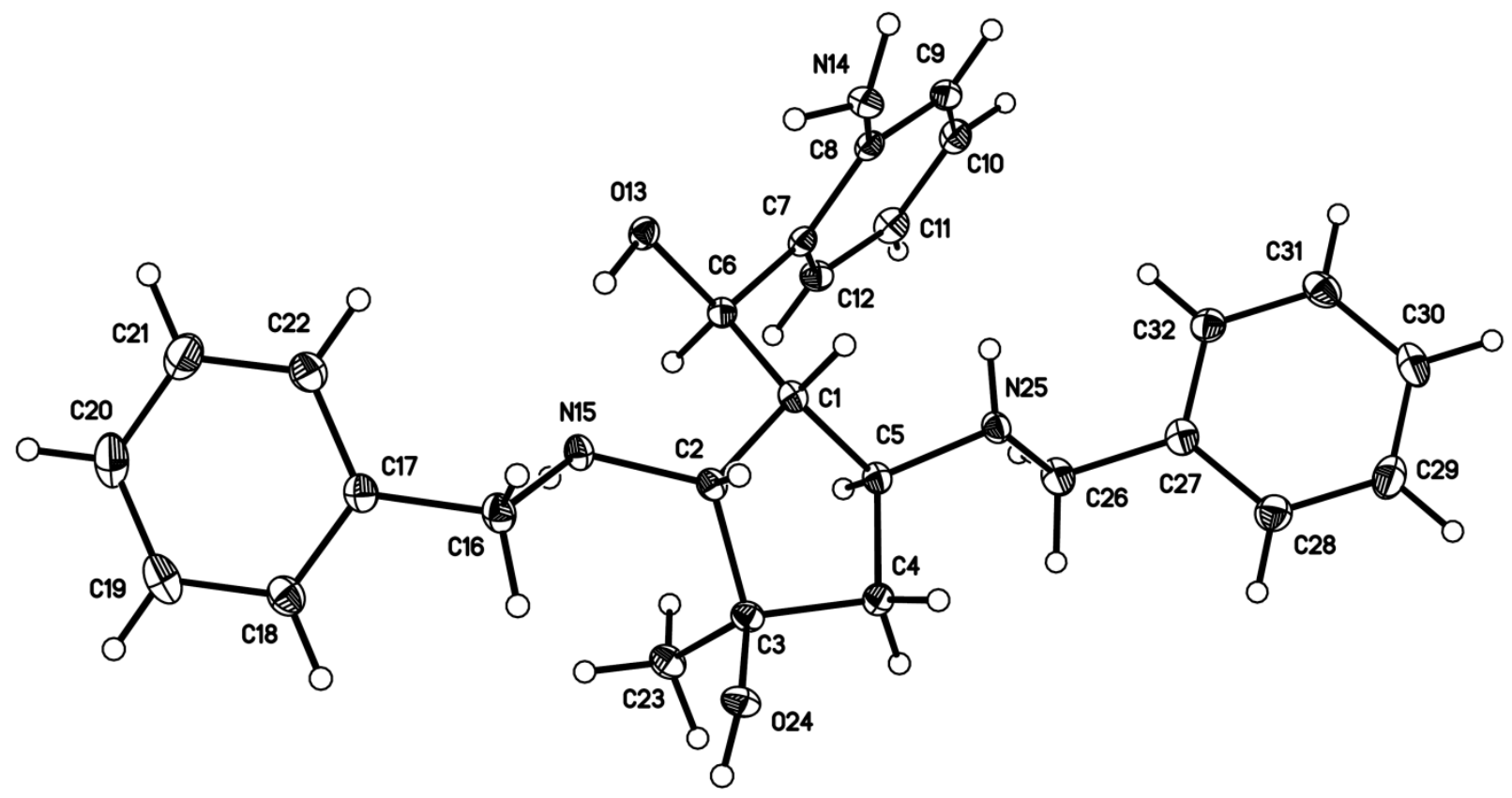

X-ray figure (CCDC 1990753, ellipsoid contour \% probability 35\%) of ent-CB 2e 
E. ${ }^{1}$ H-NMR AND ${ }^{13}$ C-NMR SPECTRA 


\section{PD170-2-H.10.1.1r}

${ }^{1} \mathrm{H}-\mathrm{NMR}$ 400MHz

in chloroform-d

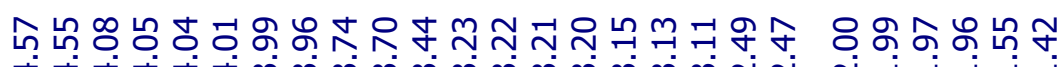

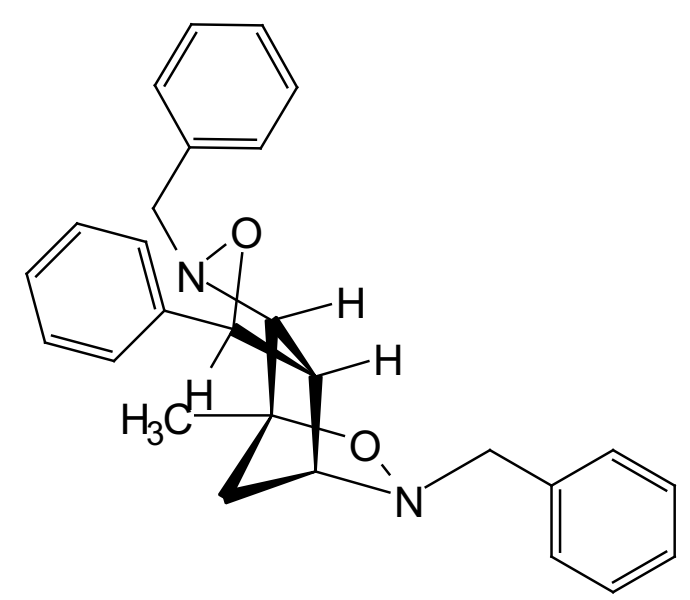

$1 a$

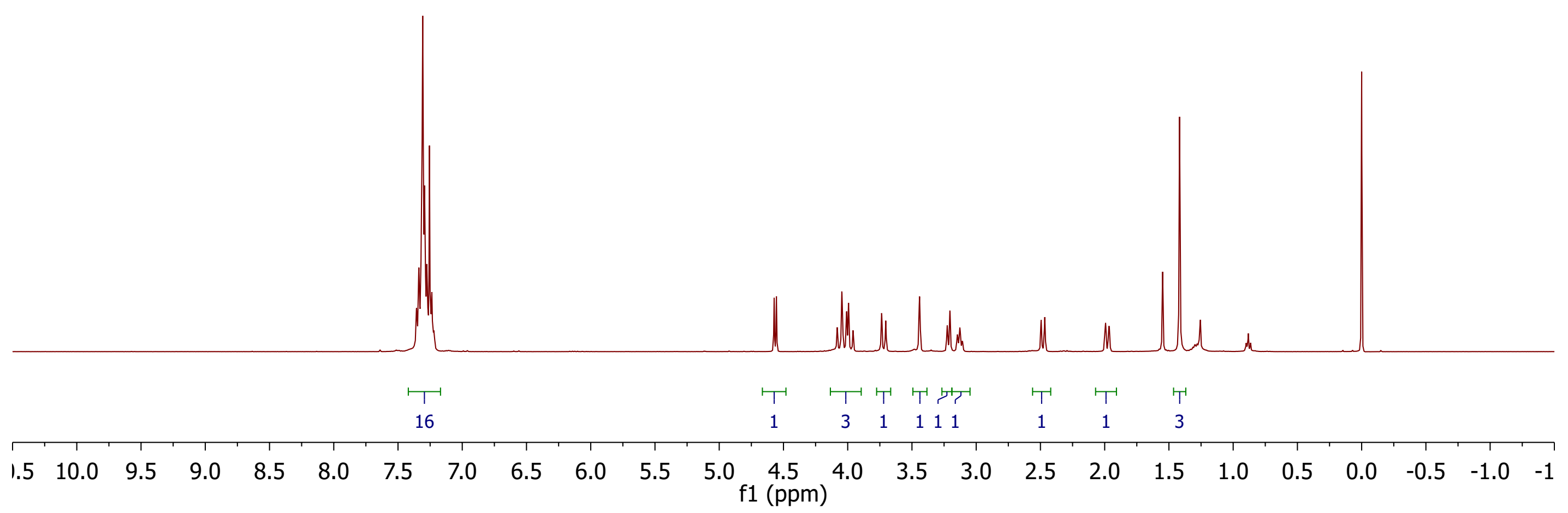


${ }^{13} \mathrm{C}-\mathrm{NMR} 100 \mathrm{MHz}$

in chloroform-d

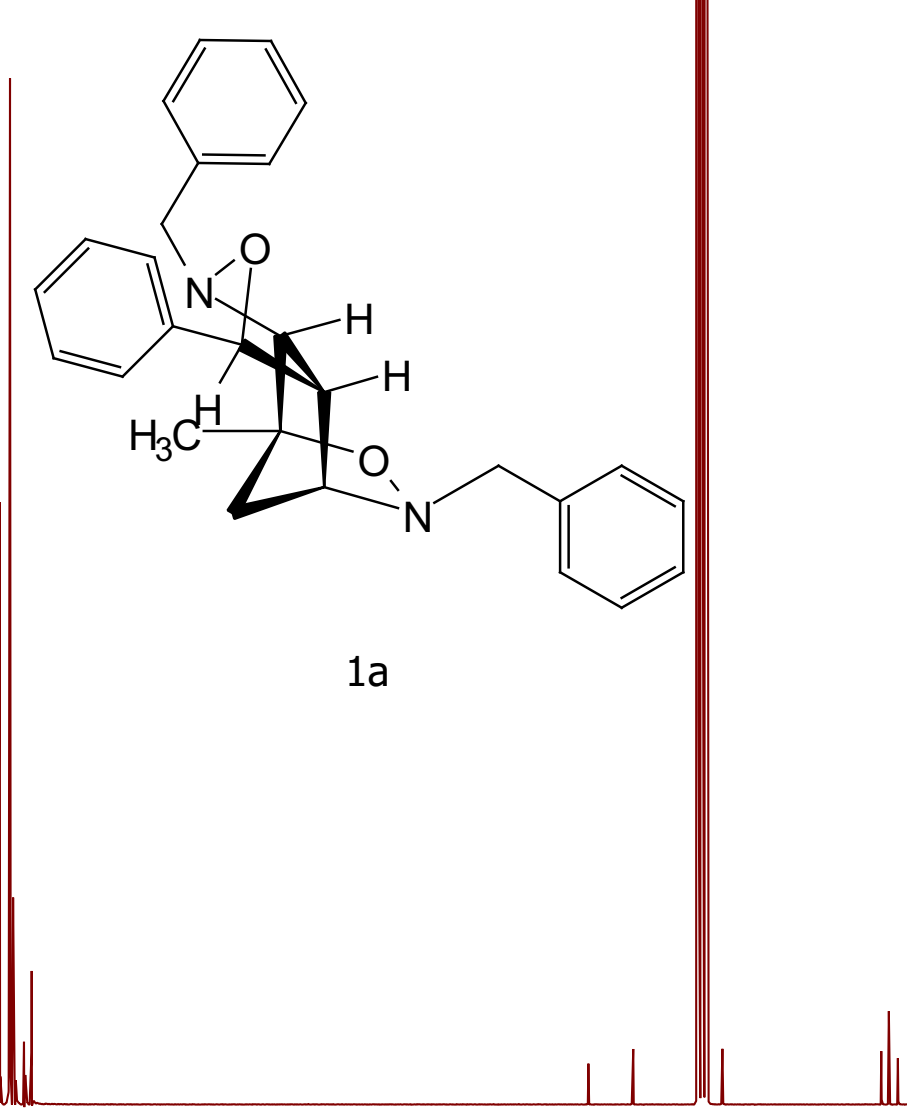

180

\begin{tabular}{|c|c|c|c|c|c|c|c|c|c|}
\hline 80 & 70 & 60 & 50 & 40 & 30 & 20 & 10 & 0 & -1 \\
\hline
\end{tabular}




\section{${ }^{1} \mathrm{H}-\mathrm{NMR} 400 \mathrm{MHz}$ \\ in chloroform-d}

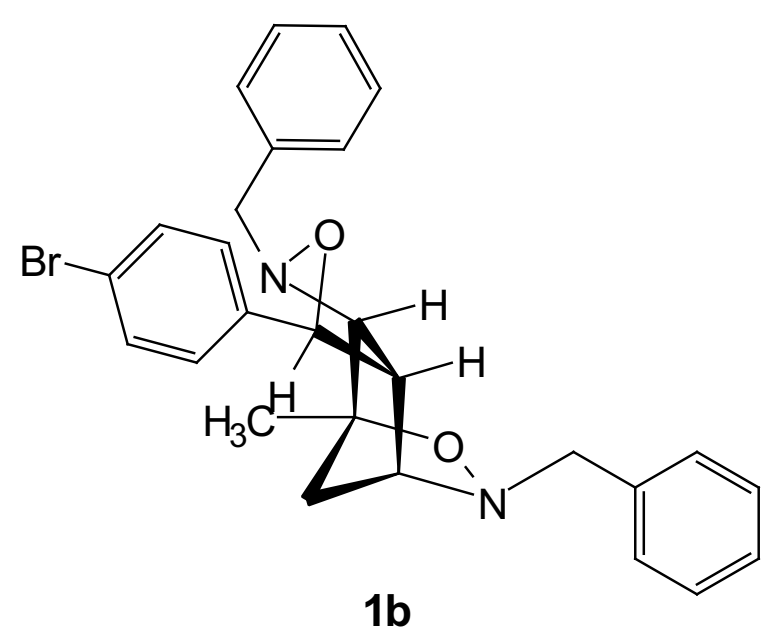

$1 b$

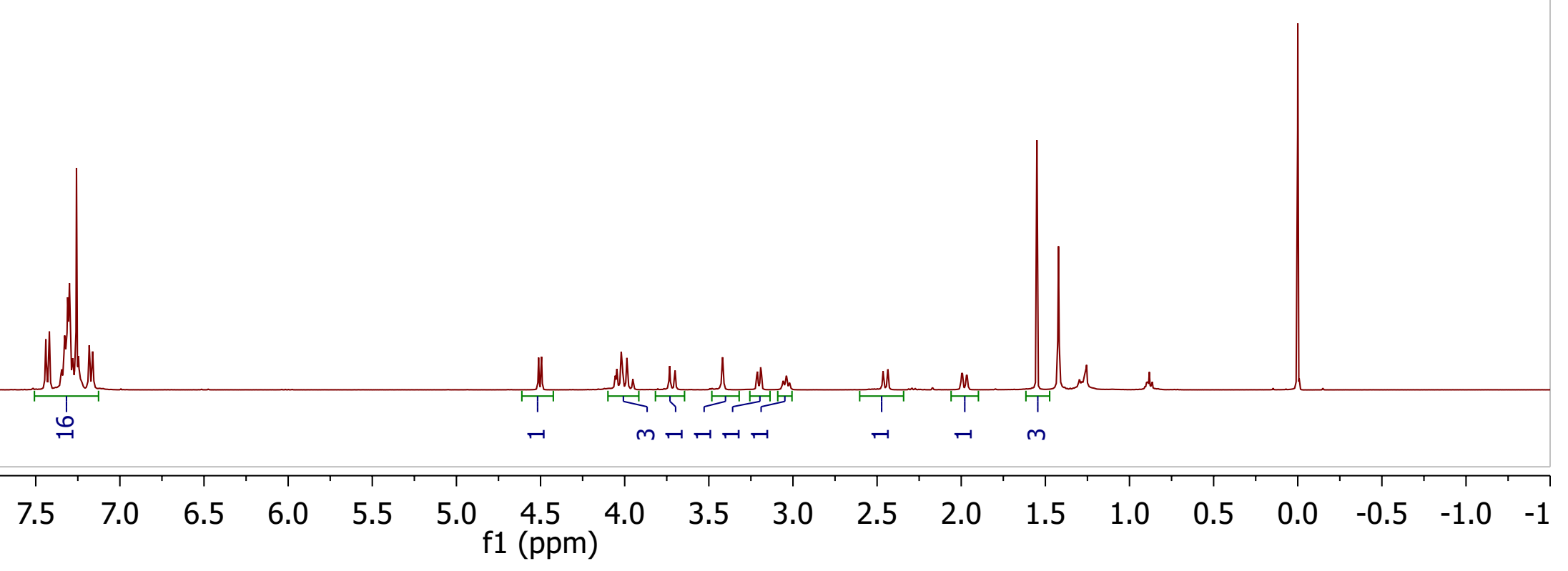




\section{PD226-2-C.12.1.1r}

${ }^{13} \mathrm{C}-\mathrm{NMR} 100 \mathrm{MHz}$ in chloroform-d

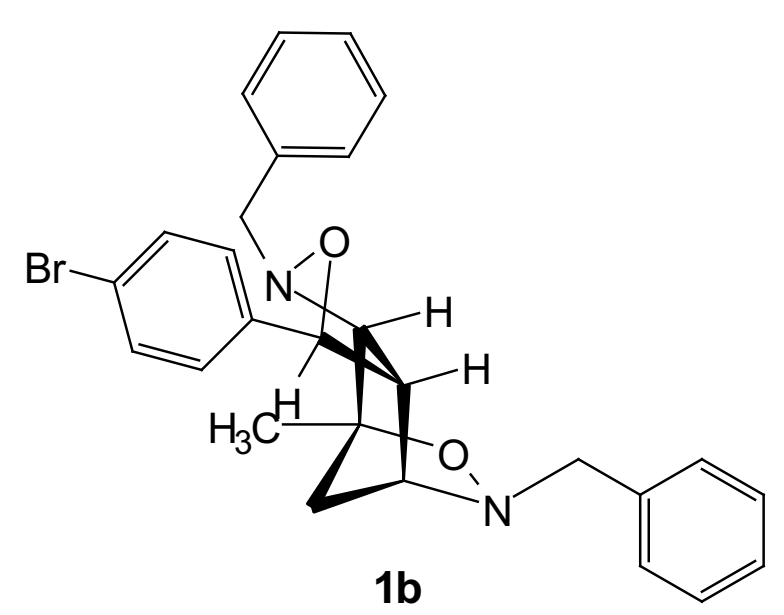

|l

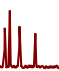




\section{${ }^{1} \mathrm{H}-\mathrm{NMR} 400 \mathrm{MHz}$}

in chloroform-d

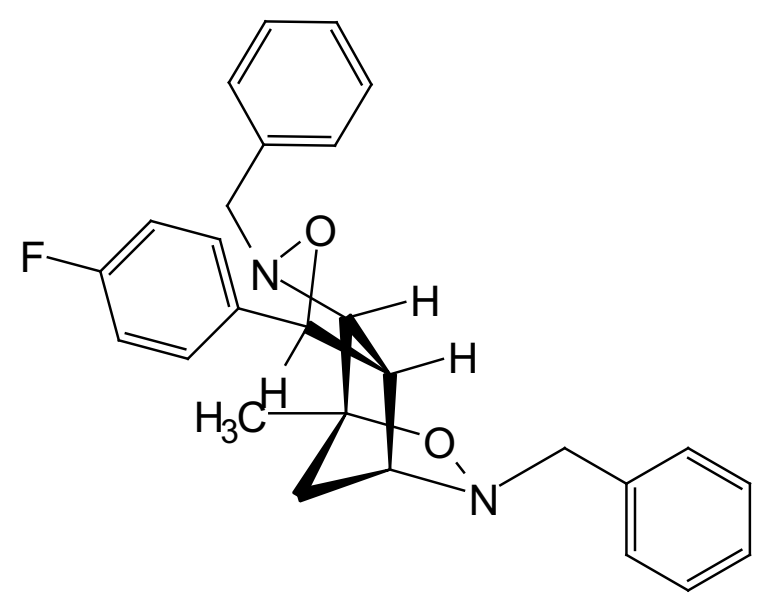

$1 \mathrm{c}$

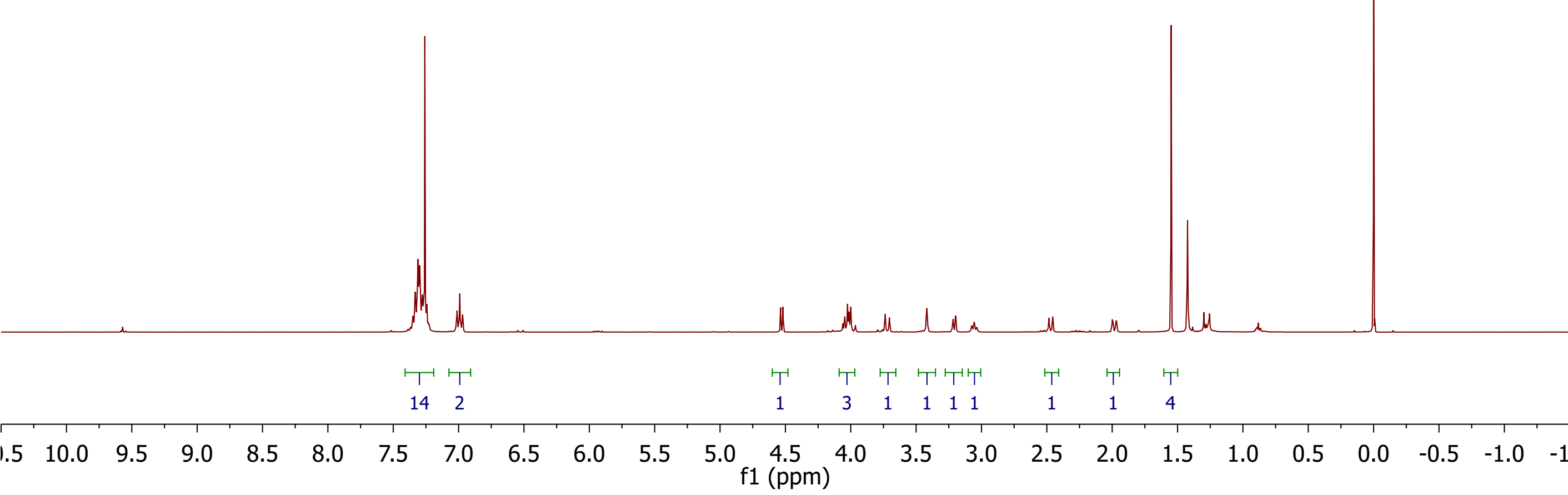


${ }^{13} \mathrm{C}-\mathrm{NMR} 100 \mathrm{MHz}$ in chloroform-d
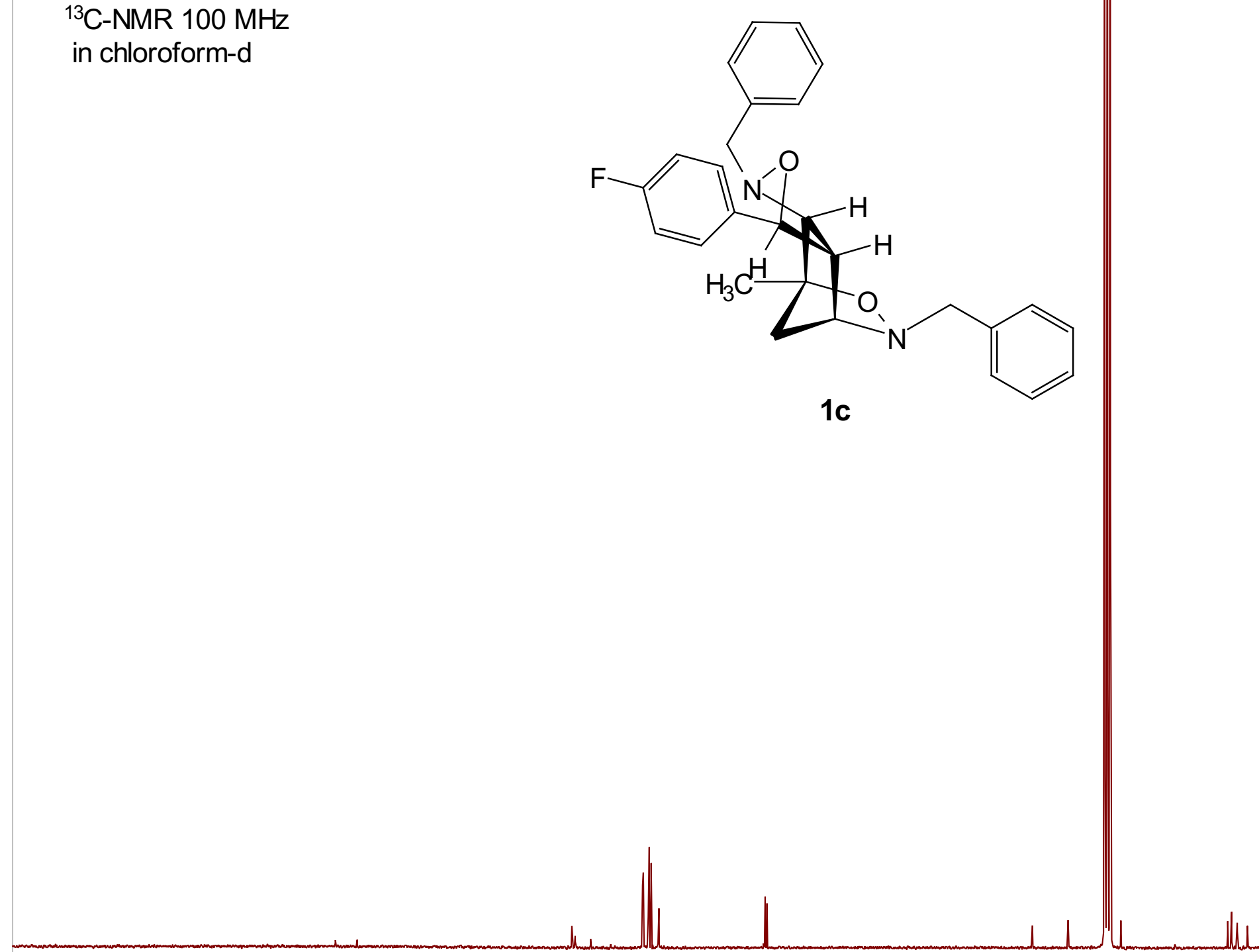


\section{${ }^{1} \mathrm{H}-\mathrm{NMR} 400 \mathrm{MHz}$ \\ in chloroform-d}
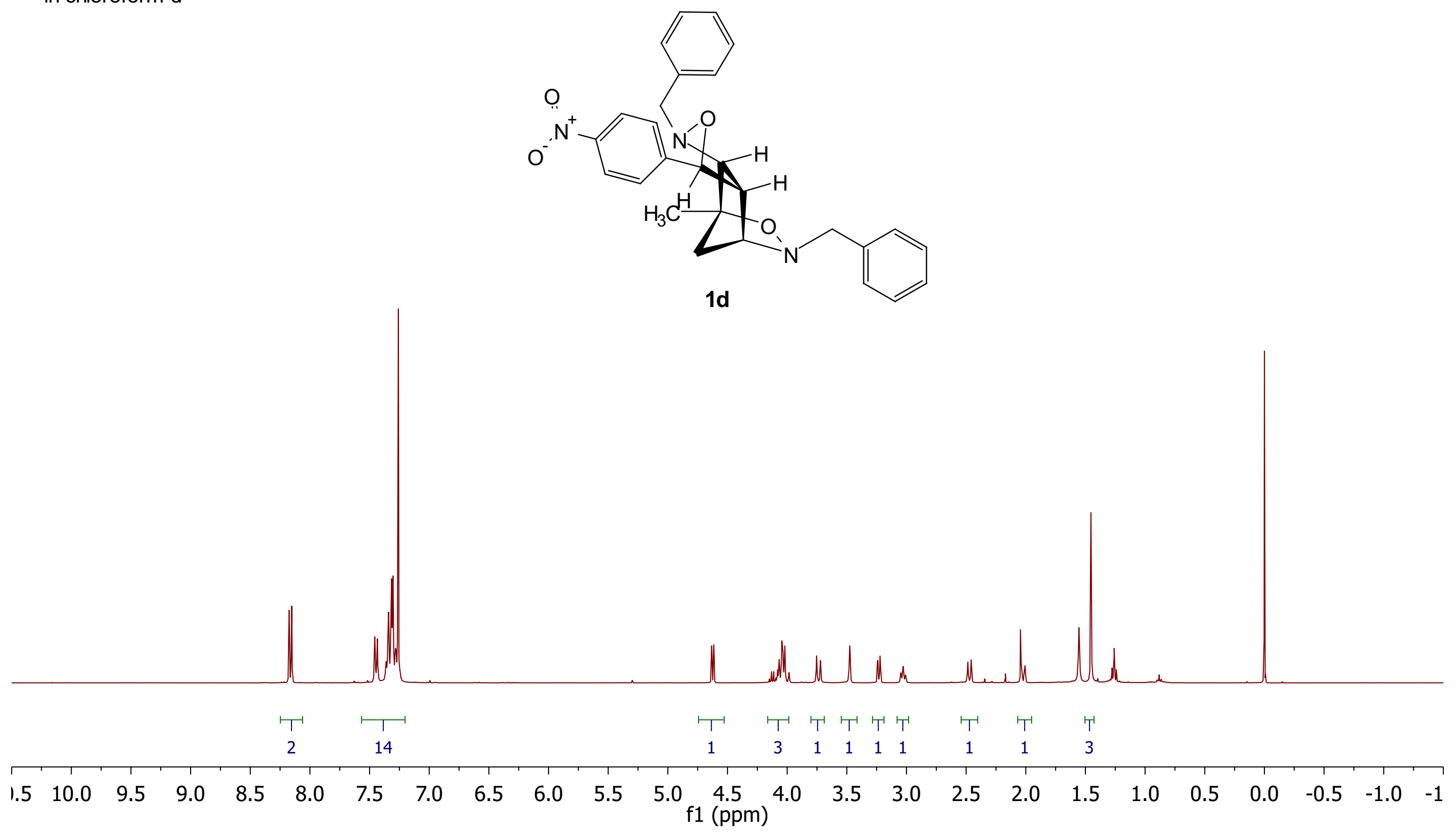
${ }^{13} \mathrm{C}-\mathrm{NMR} 100 \mathrm{MHz}$ in chloroform-d

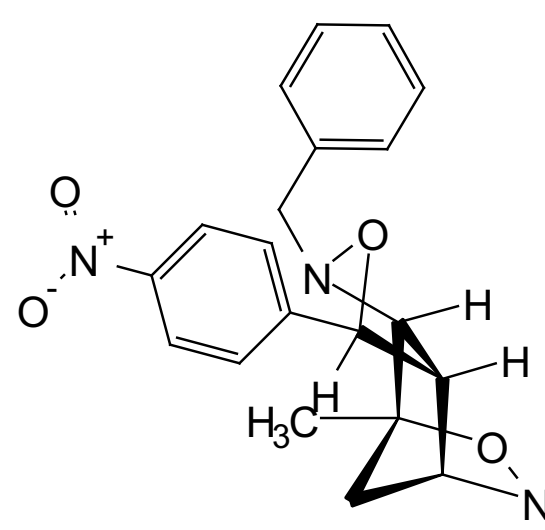

1d

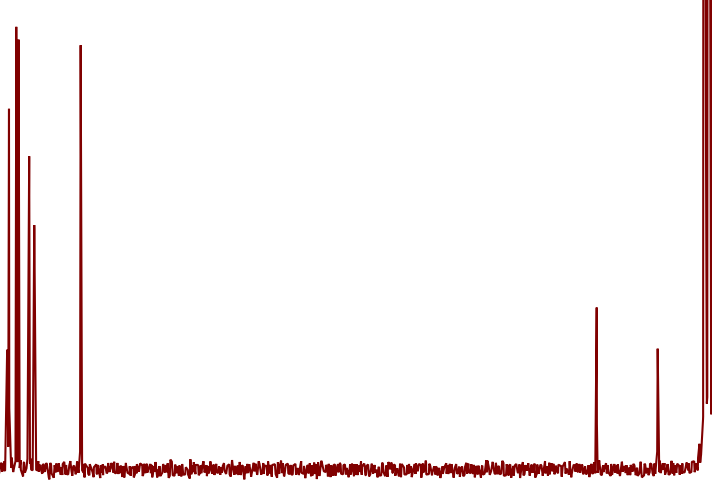

$90 \quad 180 \quad 170$

$170 \quad 160$

150

140

130

120

110

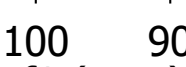

f1 (ppm)

\begin{tabular}{|c|c|c|c|c|c|c|c|c|c|}
\hline 80 & 70 & 60 & 50 & 40 & 30 & 20 & 10 & 0 & -1 \\
\hline
\end{tabular}


${ }^{1} \mathrm{H}-\mathrm{NMR} 400 \mathrm{MHz}$

in chloroform-d
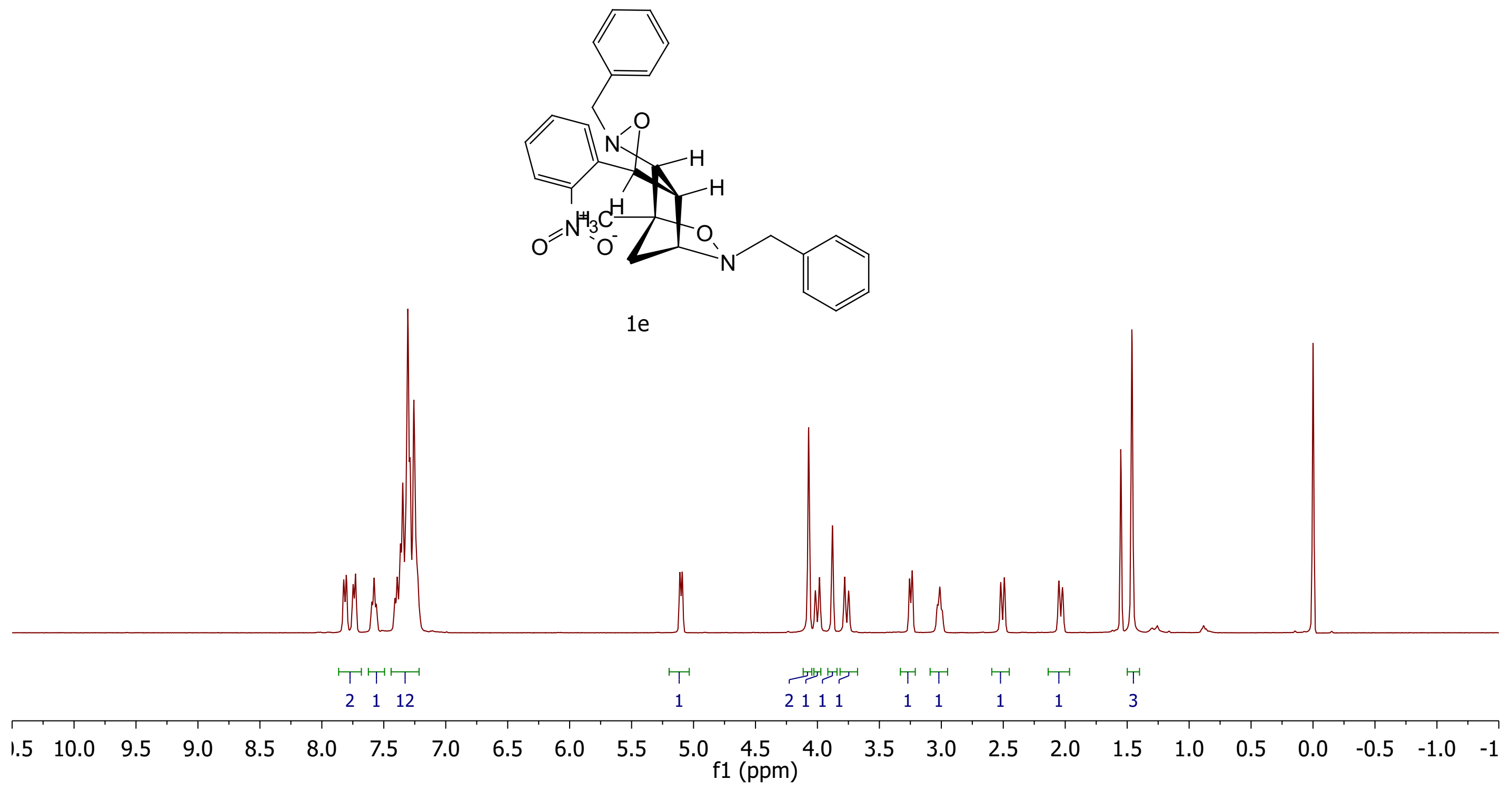
${ }^{13} \mathrm{C}-\mathrm{NMR} 100 \mathrm{MHz}$ in chloroform-d
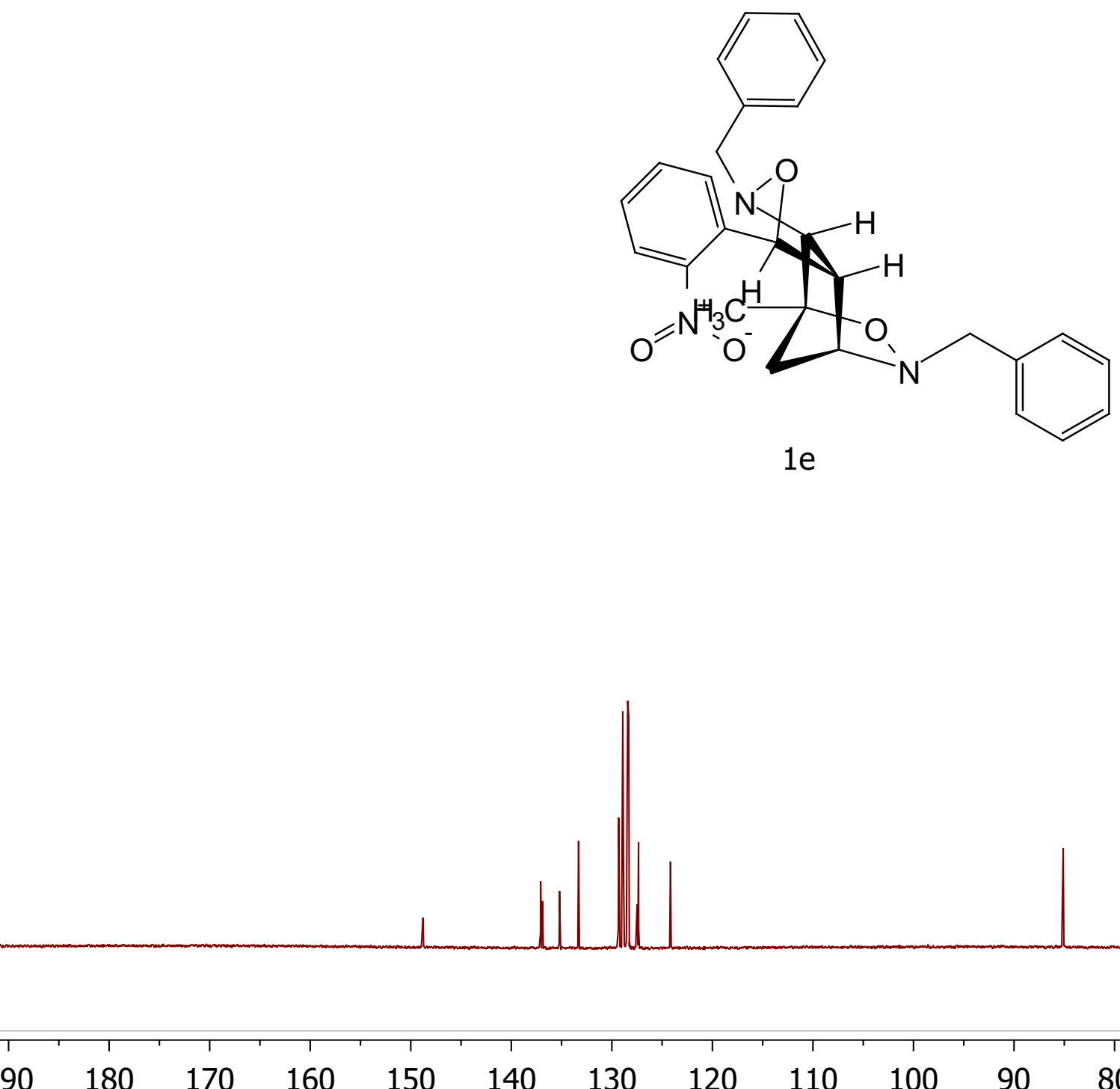

170

160

150

140

130

120

110 f1 (ppm)

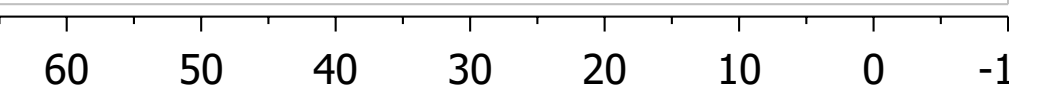




\section{${ }^{1} \mathrm{H}-\mathrm{NMR} 400 \mathrm{MHz}$}

in chloroform-d

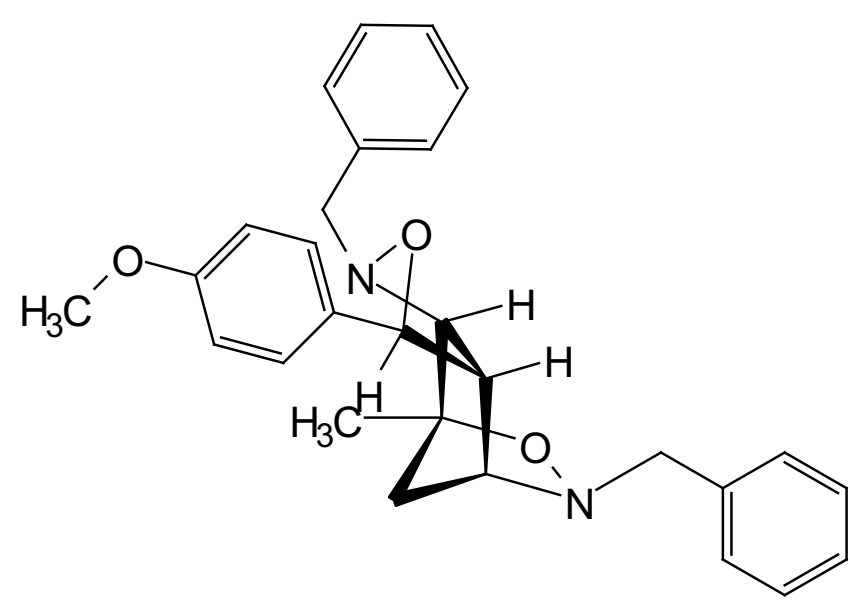

$1 f$

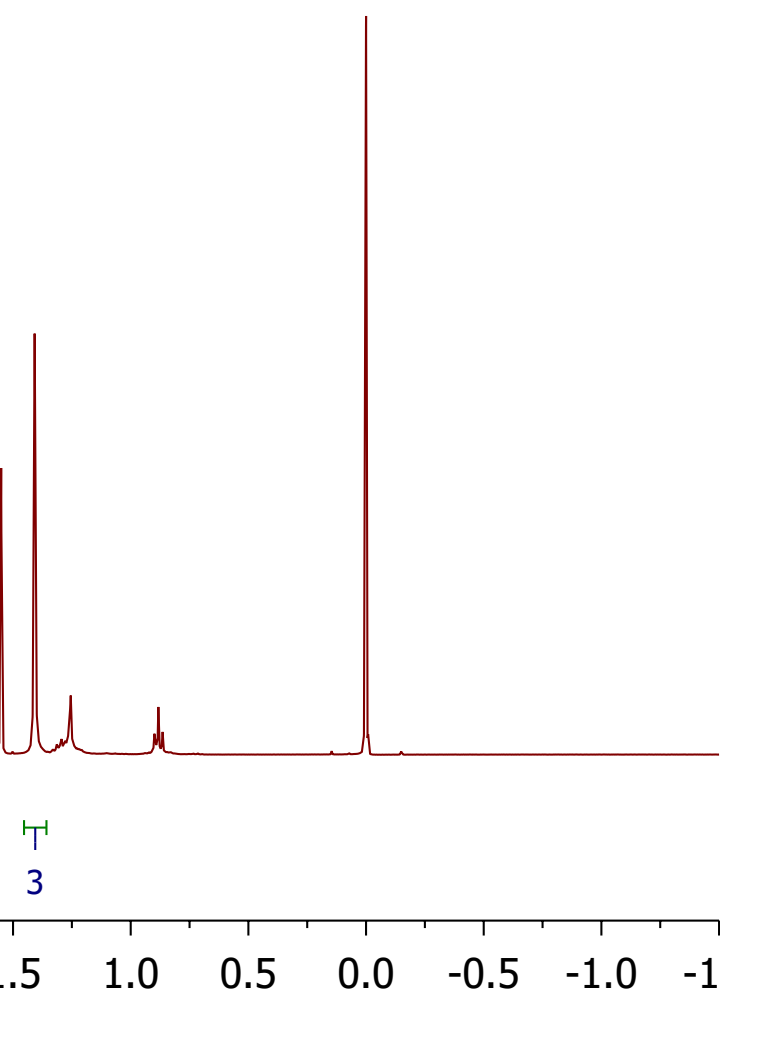




\section{PD161-2-pure-C.1.1.1r f

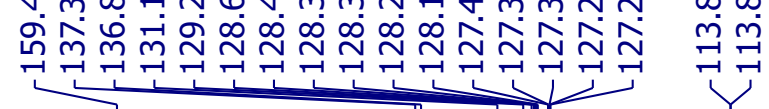

\section{${ }^{13} \mathrm{C}-\mathrm{NMR} 100 \mathrm{MHz}$}

in chloroform-d

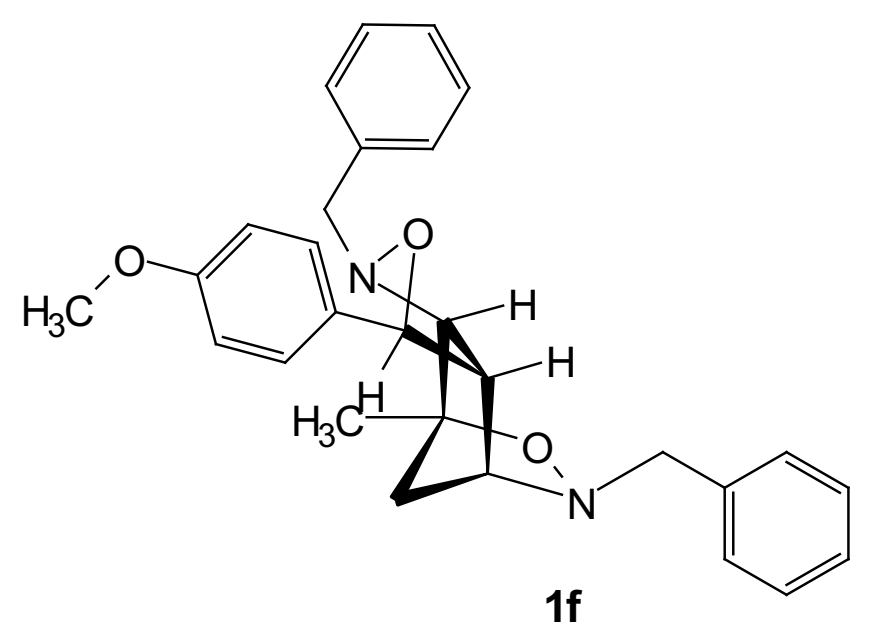

$\begin{array}{llllllllll}80 & 70 & 60 & 50 & 40 & 30 & 20 & 10 & 0 & -1\end{array}$


${ }^{1} \mathrm{H}-\mathrm{NMR} 400 \mathrm{MHz}$

in chloroform-d

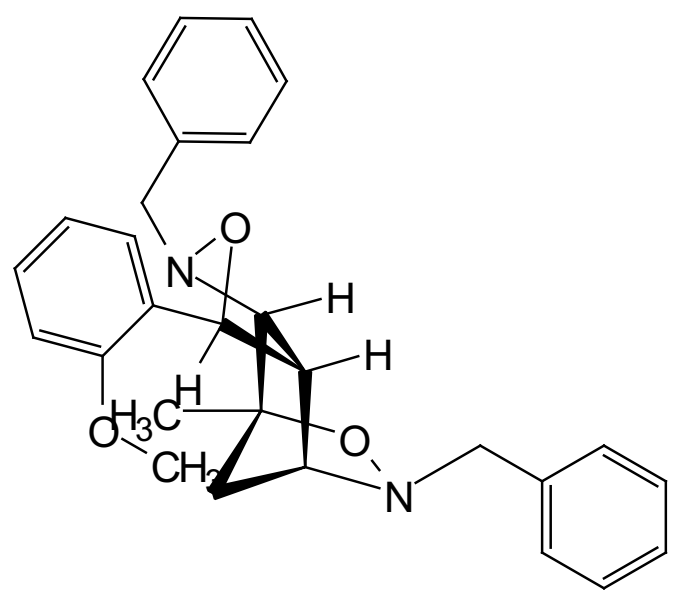

19

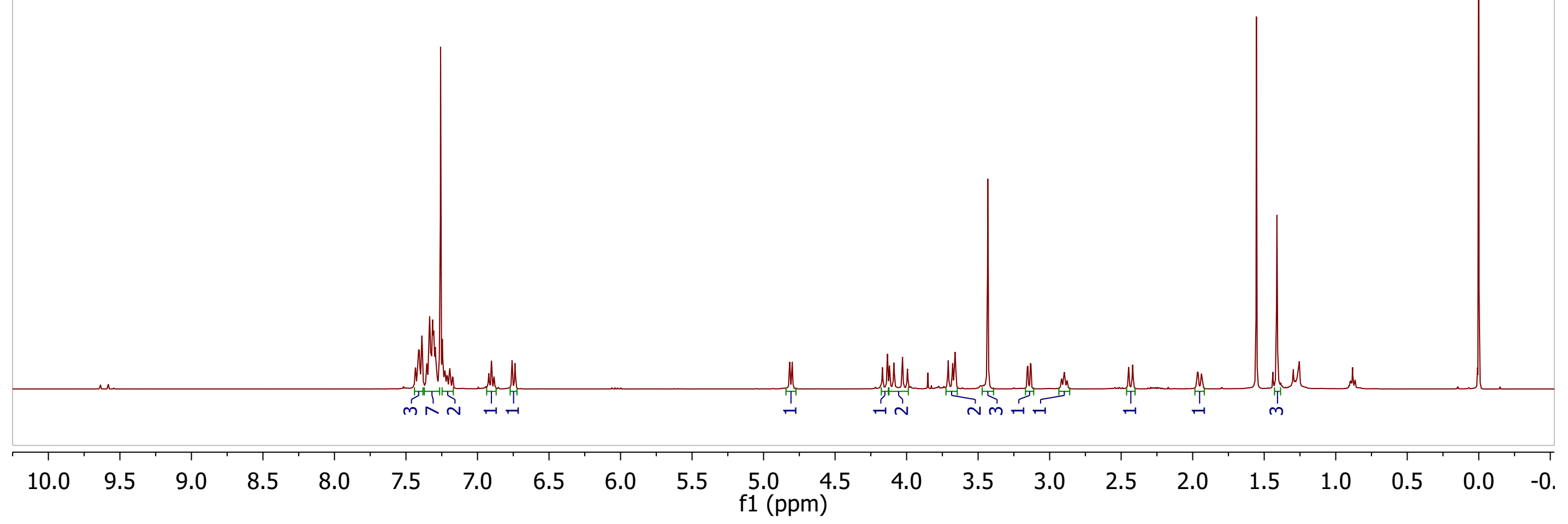


${ }^{13} \mathrm{C}-\mathrm{NMR} 100 \mathrm{MHz}$ in chloroform-d

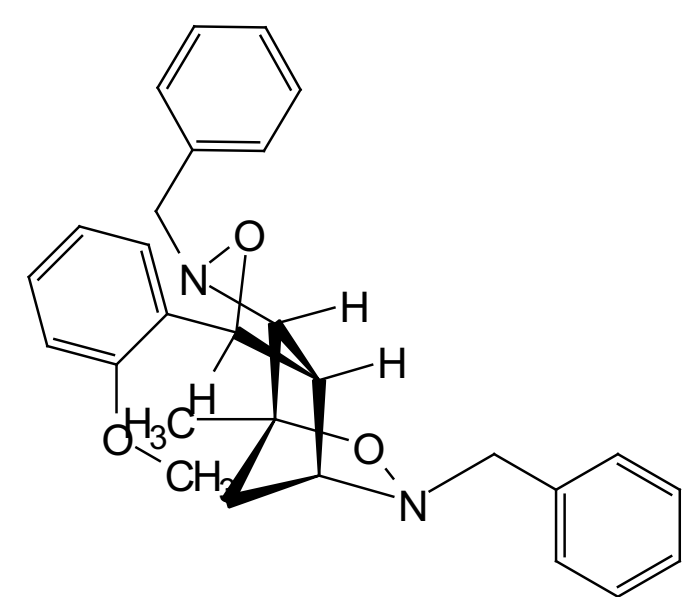

$1 \mathrm{~g}$ 


\section{${ }^{1} \mathrm{H}-\mathrm{NMR} 400 \mathrm{MHz}$}

in chloroform-d

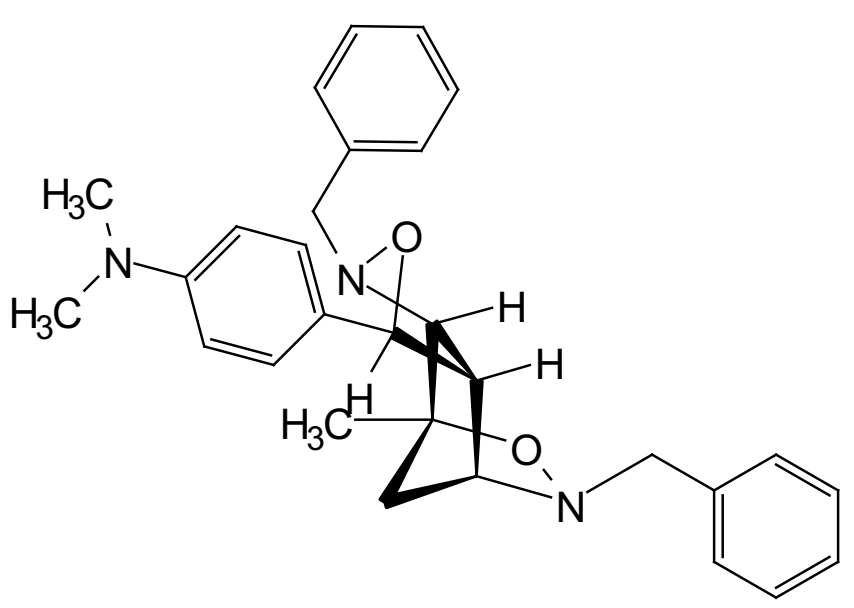

$1 \mathrm{~h}$

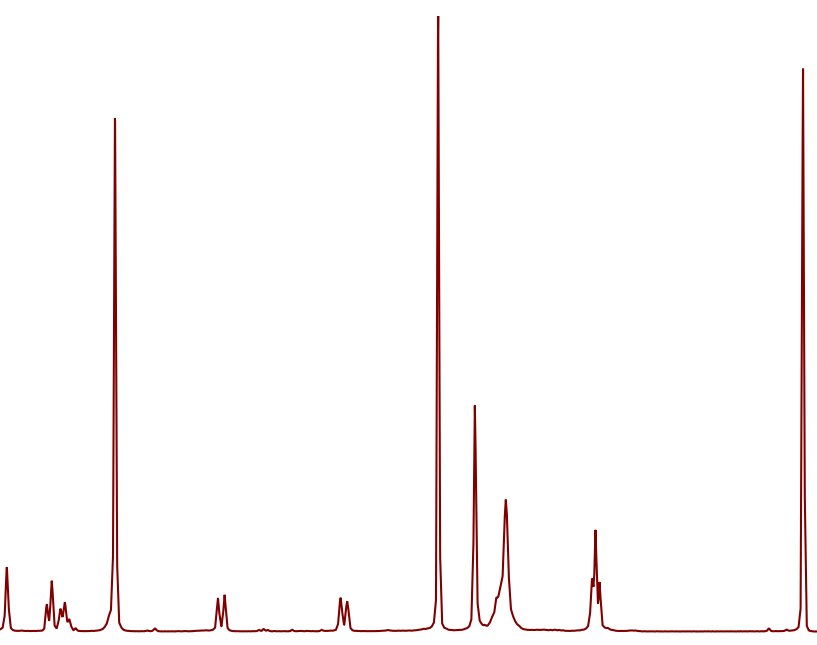

$\longmapsto \frac{1}{15}$

† म $\begin{array}{lllllllllll}1 & 3 & 1 & 1 & 2 & 6 & 1 & 1\end{array}$

\begin{tabular}{llllllllllllllllllllllllllll}
\hline .5 & 10.0 & 9.5 & 9.0 & 8.5 & 8.0 & 7.5 & 7.0 & 6.5 & 6.0 & 5.5 & 5.0 & $\begin{array}{l}4.5 \\
\text { f }\end{array}$ & 4.0 & 3.5 & 3.0 & 2.5 & 2.0 & 1.5 & 1.0 & 0.5 & 0.0 & -0.5 & -1.0 & -1
\end{tabular}


acqfil

BEA-2018-145-P-Carbon

${ }^{13} \mathrm{C}-\mathrm{NMR} 100 \mathrm{MHz}$

in chloroform-d

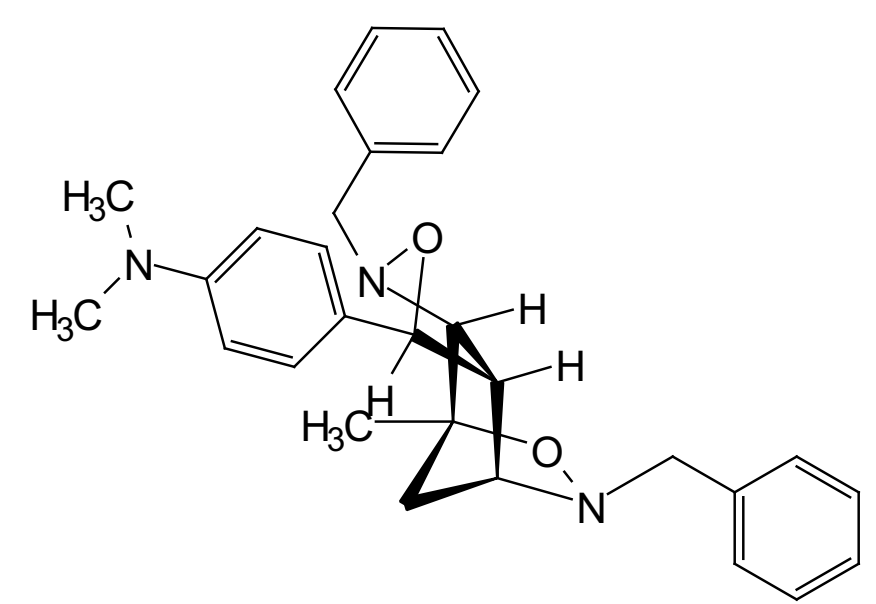

$1 \mathrm{~h}$

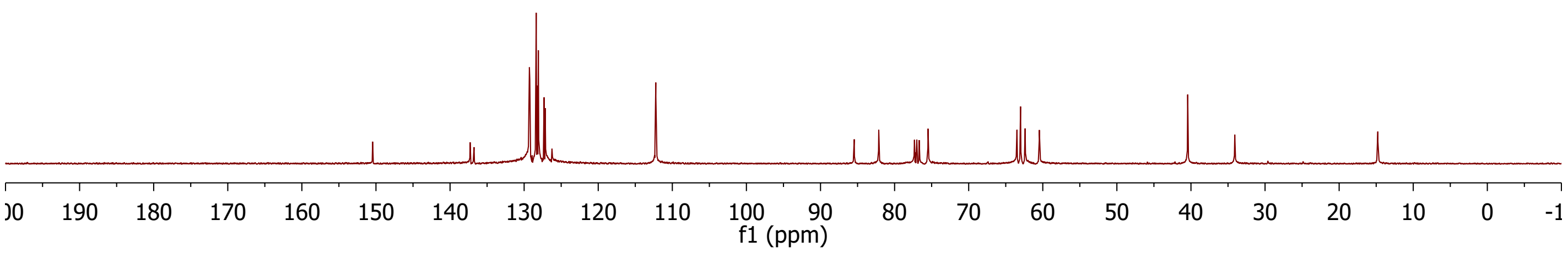


${ }^{13} \mathrm{C}-\mathrm{NMR} 100 \mathrm{MHz}$ in chloroform-d

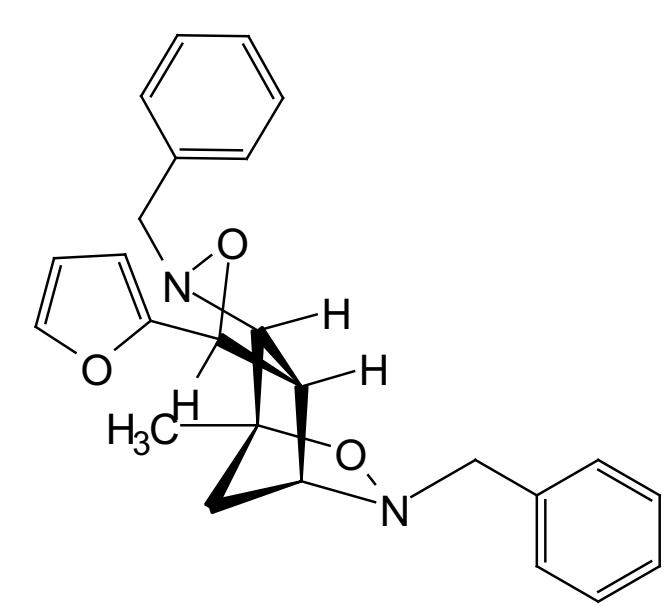

$1 i$

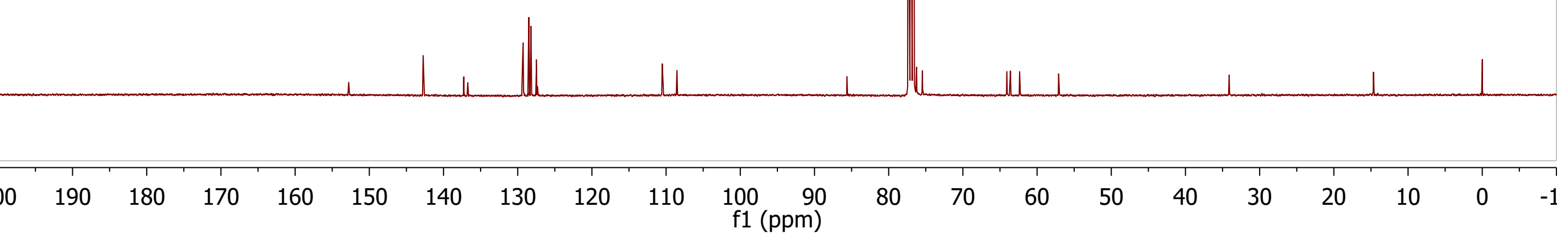




\section{${ }^{1} \mathrm{H}-\mathrm{NMR} 400 \mathrm{MHz}$} in chloroform-d

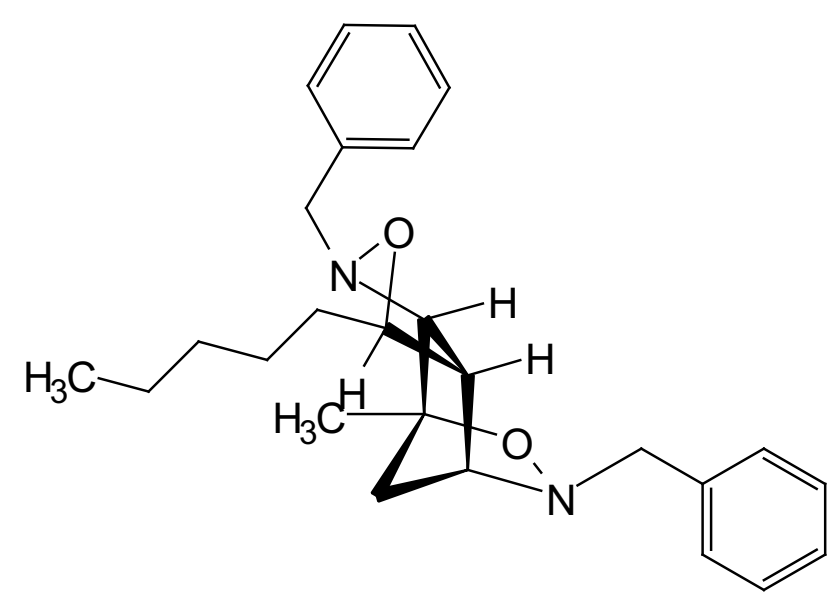

$1 \mathrm{j}$

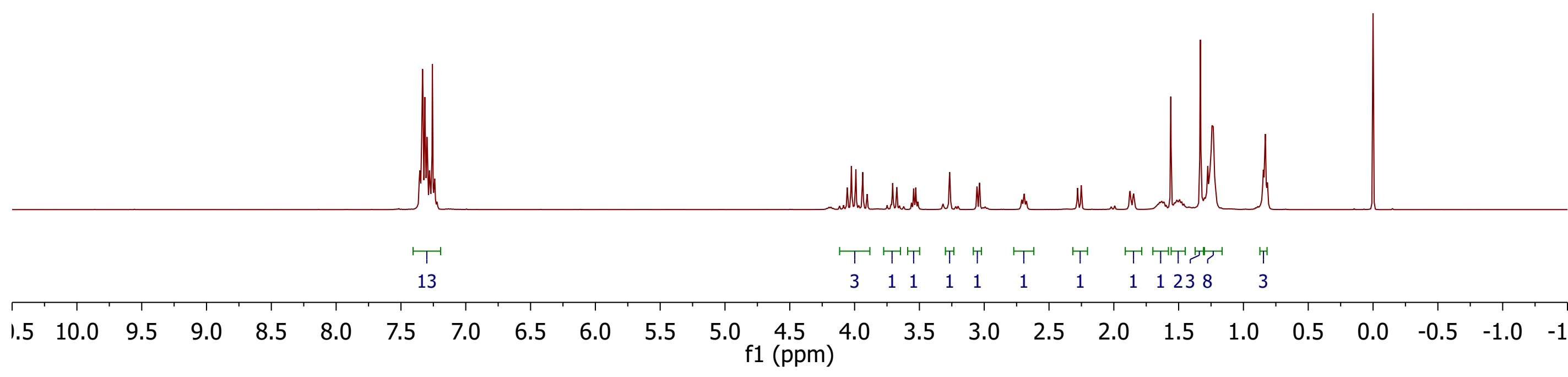




\section{PD213-2-C.12.1.1r}

${ }^{13} \mathrm{C}-\mathrm{NMR} 100 \mathrm{MHz}$

in chloroform-d

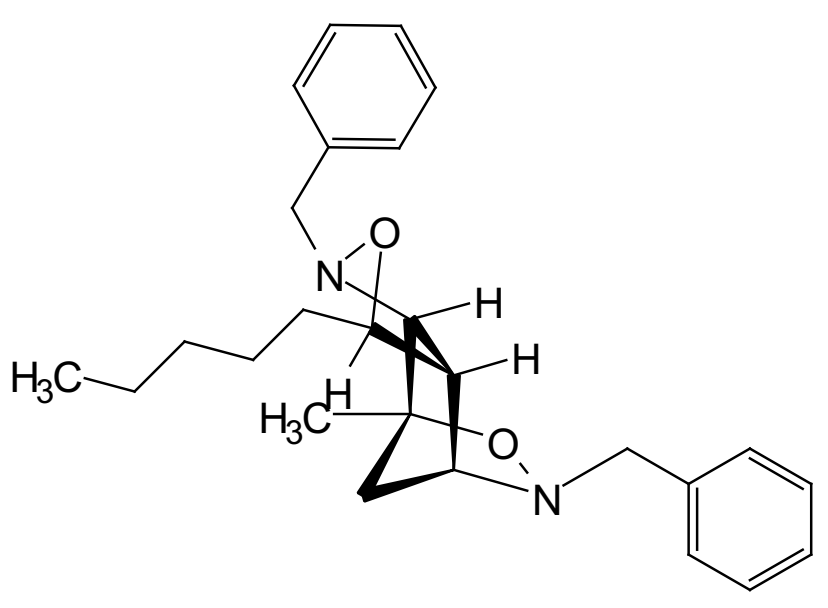

1j
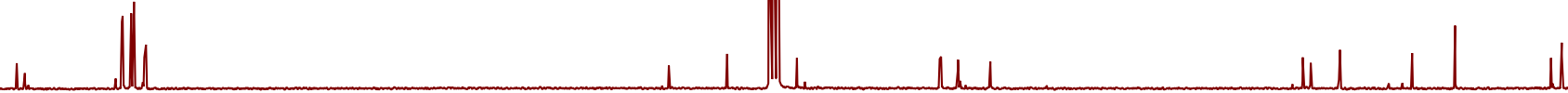

\begin{tabular}{|c|c|c|c|c|c|c|c|c|c|}
\hline 1 & ${ }_{7}^{1}$ & 10 & ${ }_{1}$ & 1 & 20 & ${ }_{1}$ & $T_{1}$ & 1 & 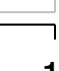 \\
\hline
\end{tabular}


${ }^{1} \mathrm{H}-\mathrm{NMR} 400 \mathrm{MHz}$

in chloroform-d

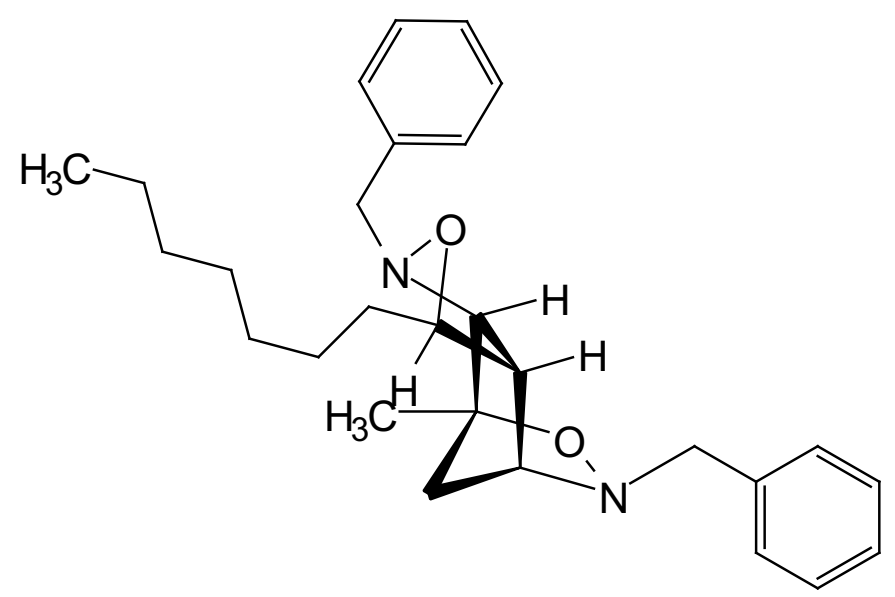

$1 \mathrm{k}$
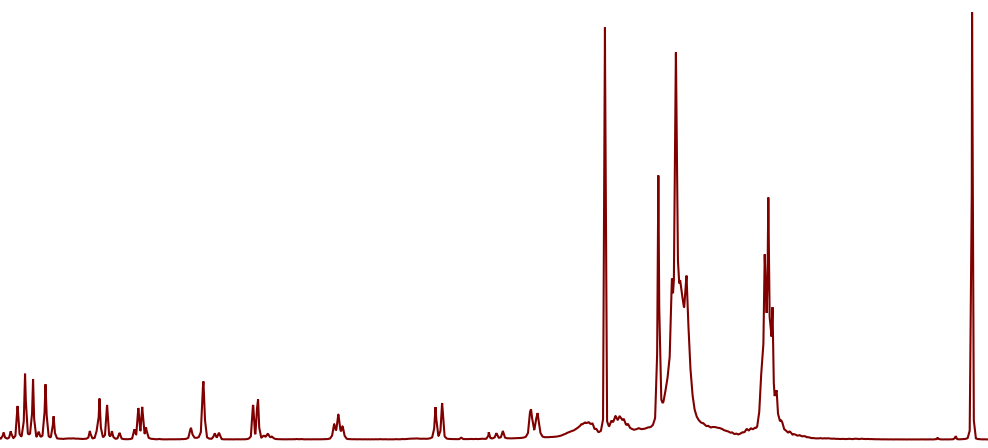

$T^{\top}$

† T

$\begin{array}{llllllllllll}3 & 1 & 1 & 1 & 1 & 1 & 1 & 11 & 3 & 1 & 15 & 6\end{array}$ 


\section{PD213-2-C.12.1.1r}

${ }^{13} \mathrm{C}-\mathrm{NMR} 100 \mathrm{MHz}$ in chloroform-d

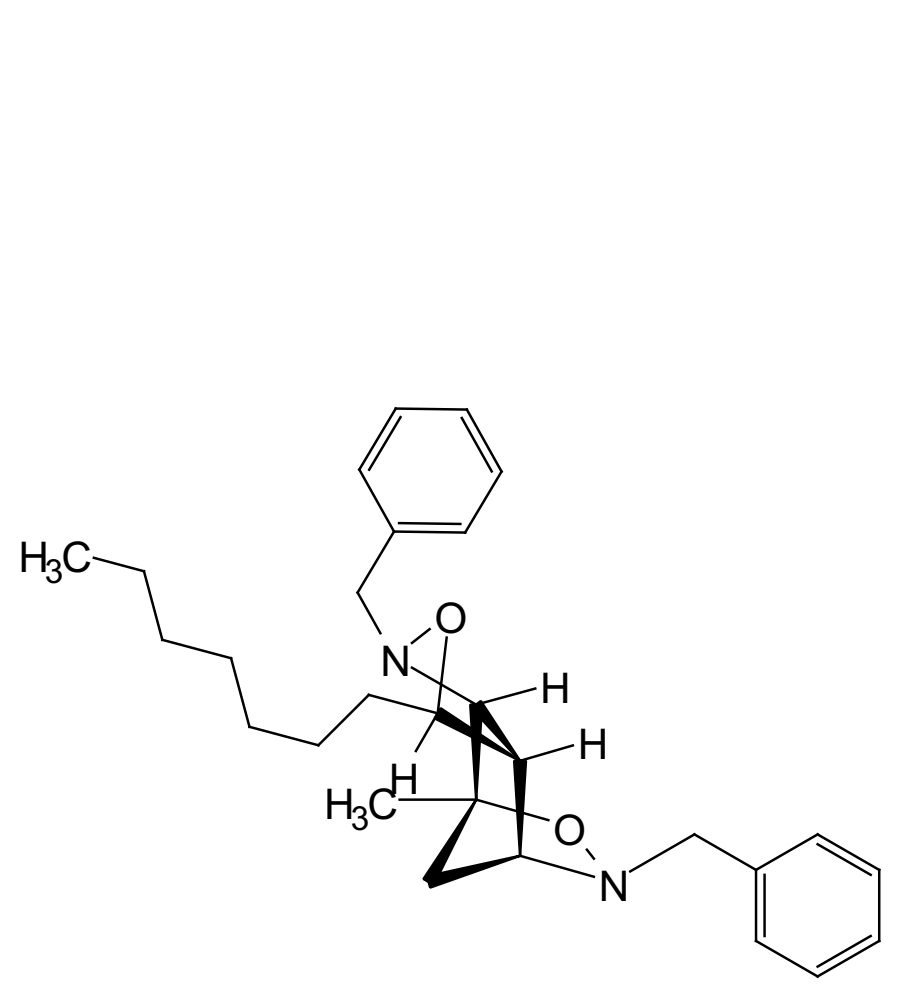

$1 \mathrm{k}$
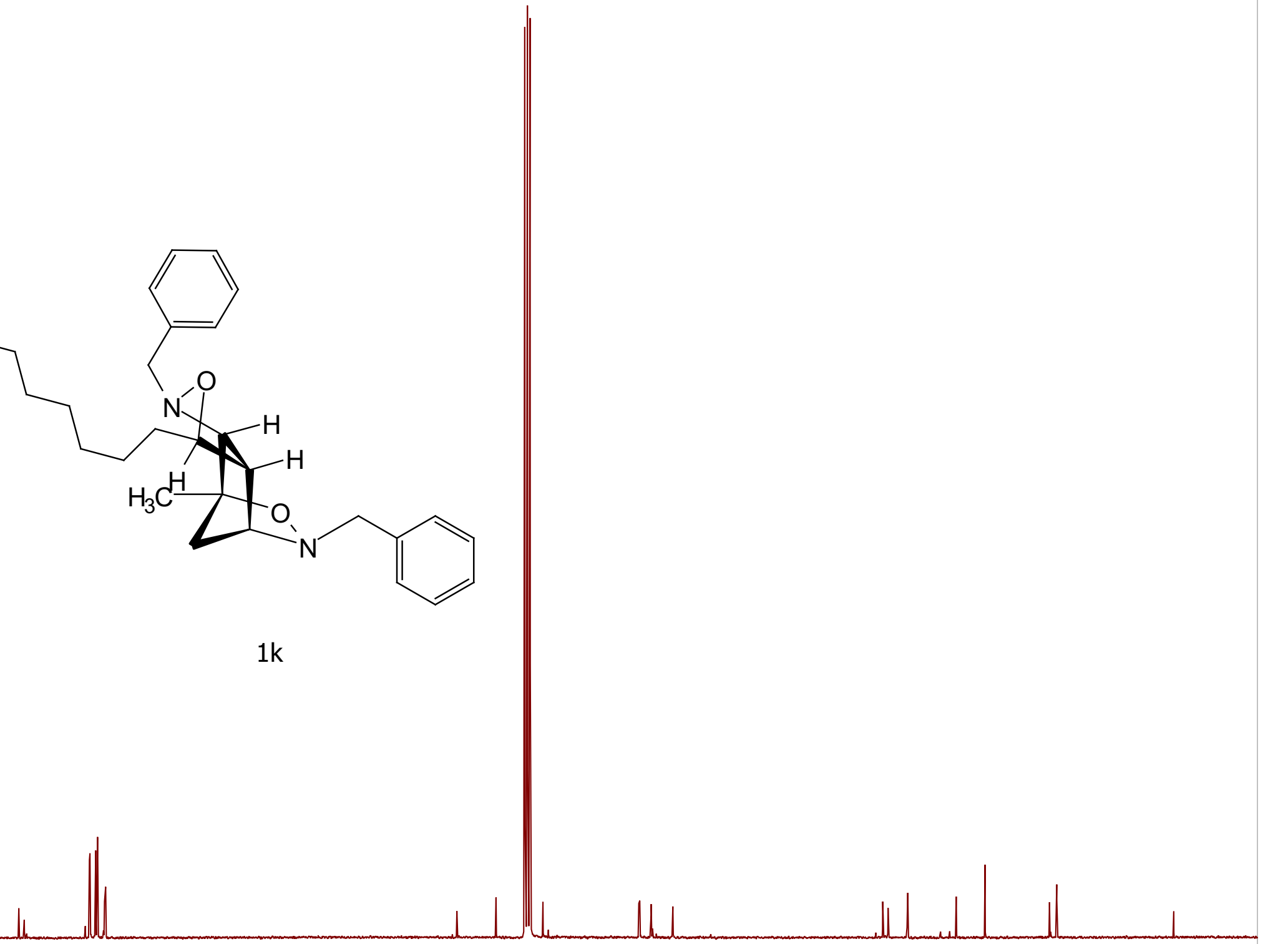

\begin{tabular}{|c|c|c|c|c|c|c|c|c|c|}
\hline 1 & ${ }_{1}$ & $I_{1}$ & 1 & 1 & 1 & 1 & 1 & $T_{1}$ & 5 \\
\hline
\end{tabular}




\section{${ }^{1} \mathrm{H}-\mathrm{NMR} 400 \mathrm{MHz}$}

in chloroform-d
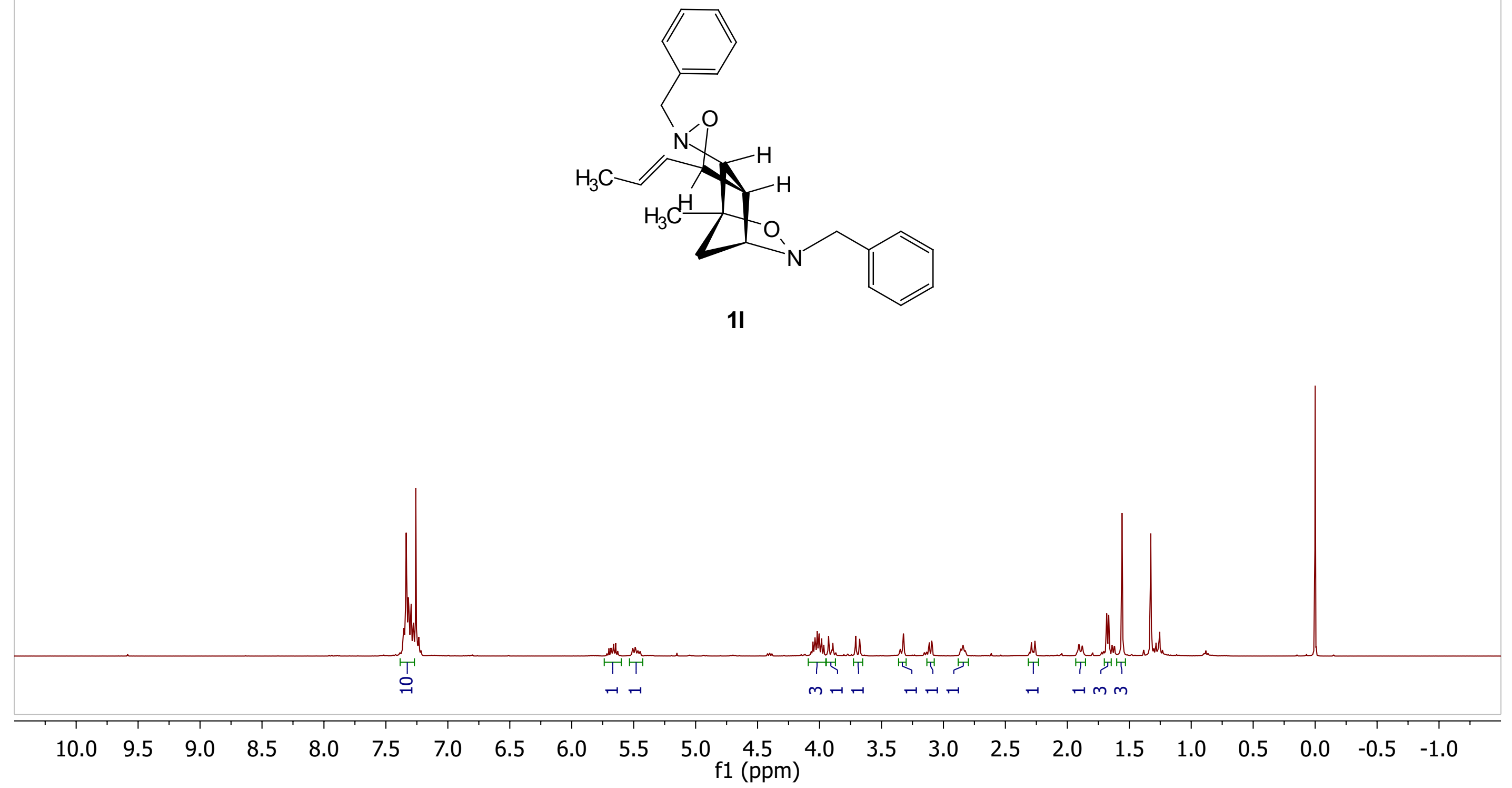


\section{PD241-2-C.12.1.1r}

${ }^{13} \mathrm{C}-\mathrm{NMR} 100 \mathrm{MHz}$ in chloroform-d

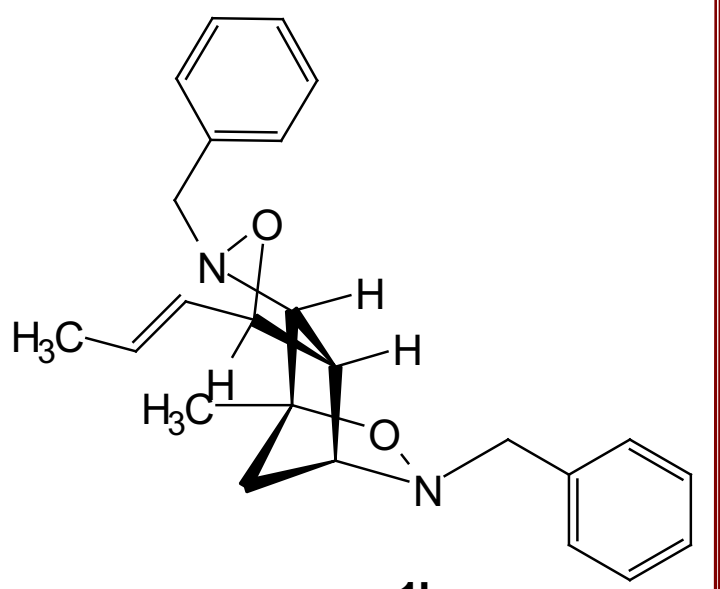

11 
${ }^{1} \mathrm{H}-\mathrm{NMR} 400 \mathrm{MHz}$

in chloroform-d
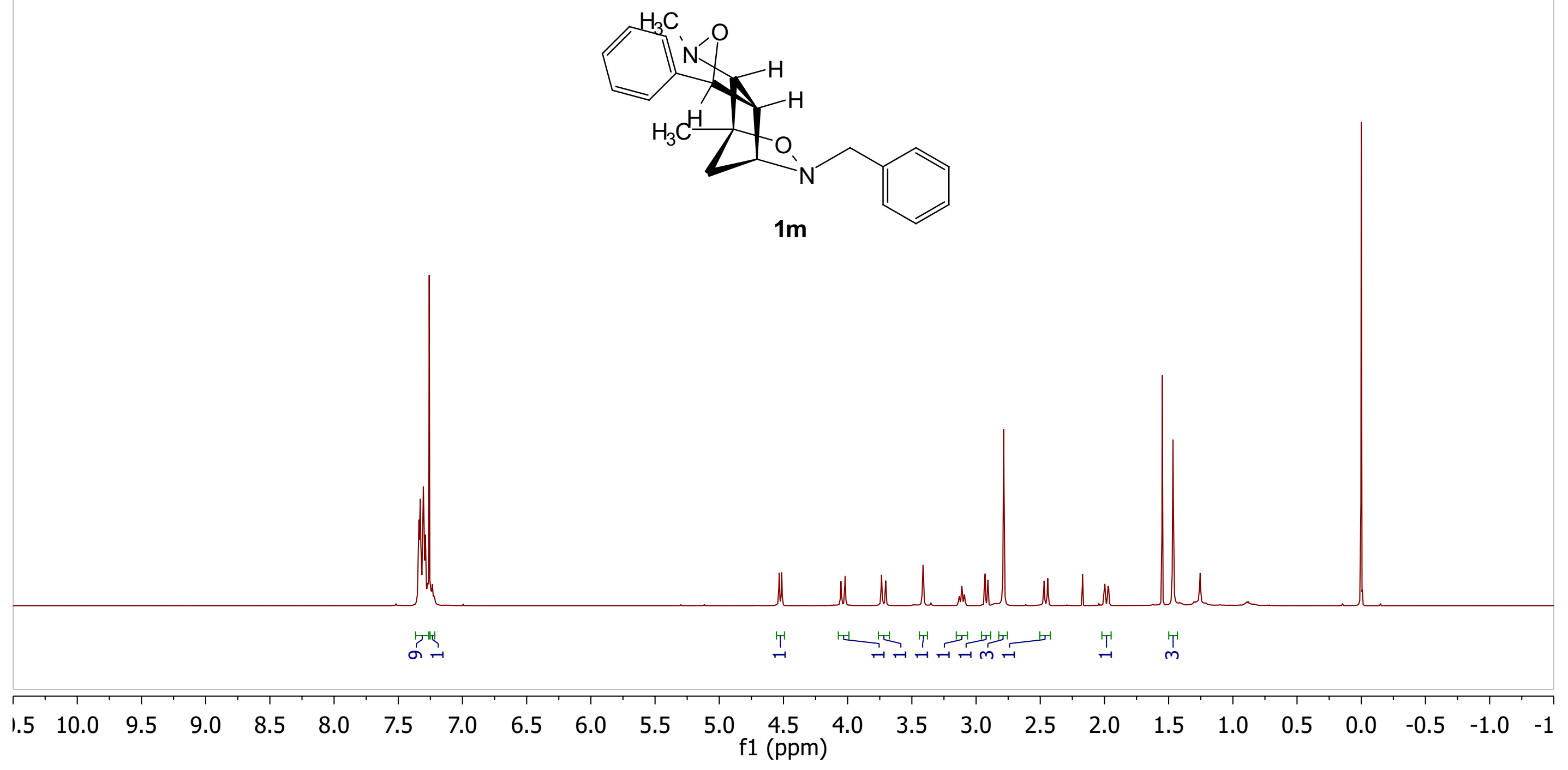
${ }^{13} \mathrm{C}-\mathrm{NMR} 100 \mathrm{MHz}$ in chloroform-d

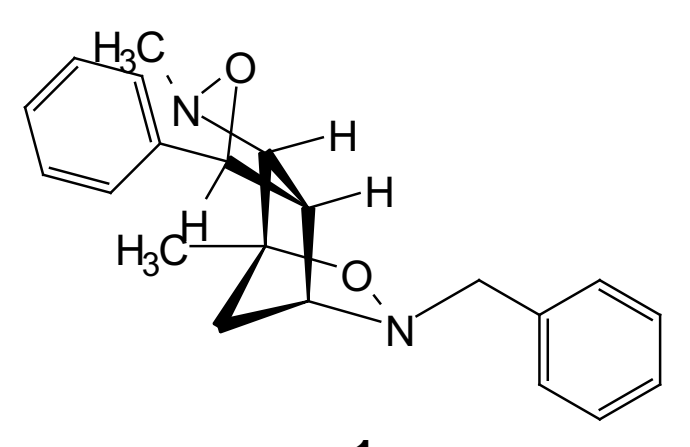

$1 \mathrm{~m}$
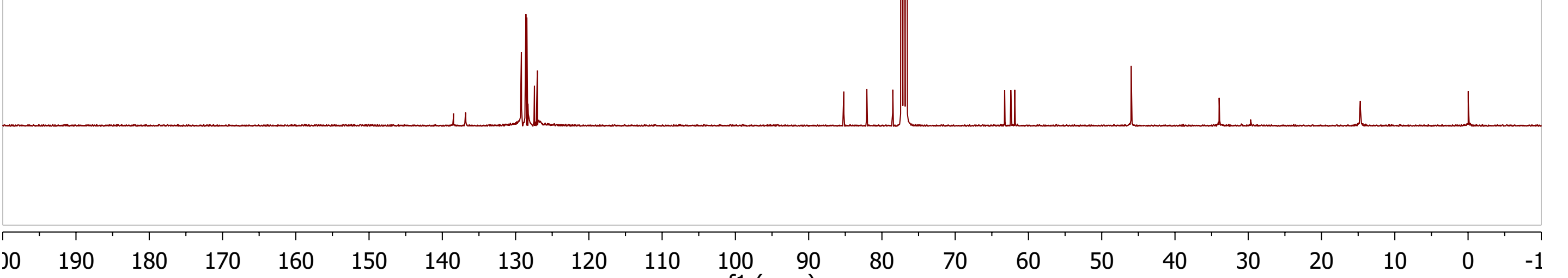
${ }^{1} \mathrm{H}-\mathrm{NMR} 400 \mathrm{MHz}$

in chloroform-d
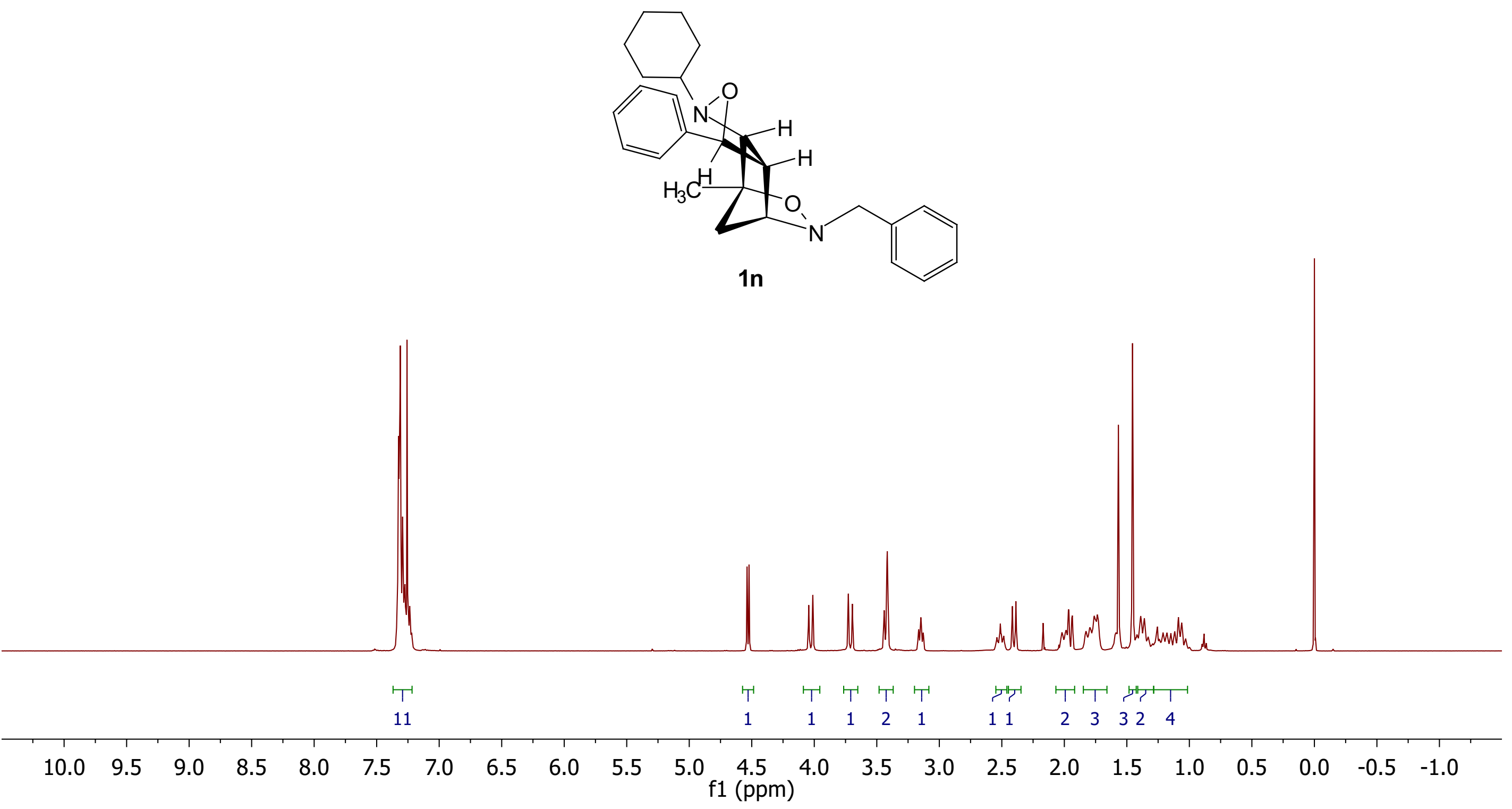


\section{PD165-1-C.12.1.1r}

${ }^{13} \mathrm{C}-\mathrm{NMR} 100 \mathrm{MHz}$

in chloroform-d

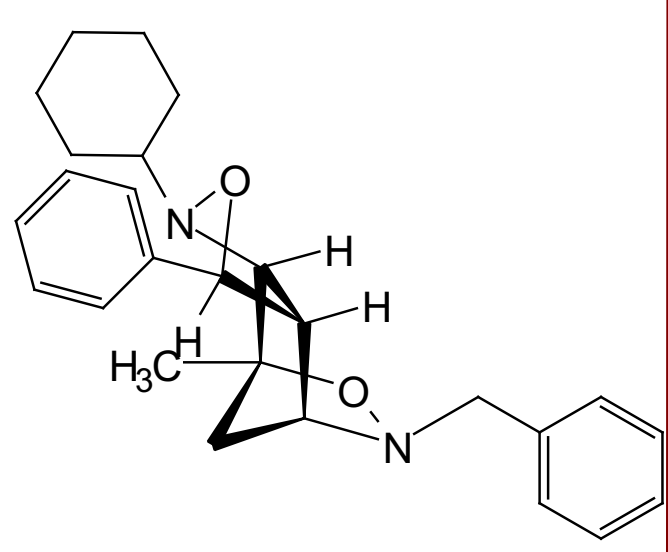

1n
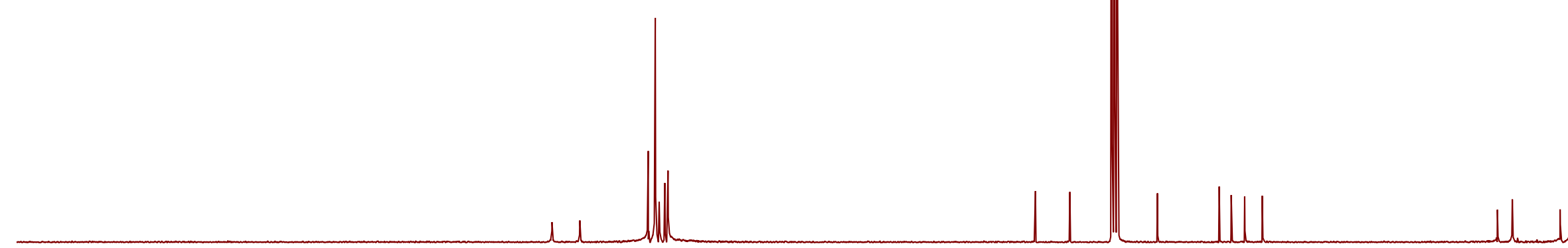

80

70

$60 \quad 50$

50

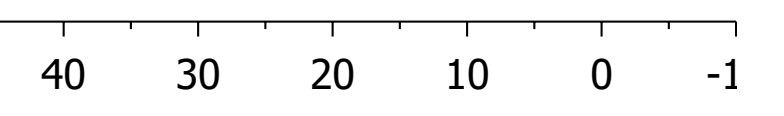




\section{${ }^{1} \mathrm{H}-\mathrm{NMR} 400 \mathrm{MHz}$}

in chloroform-d
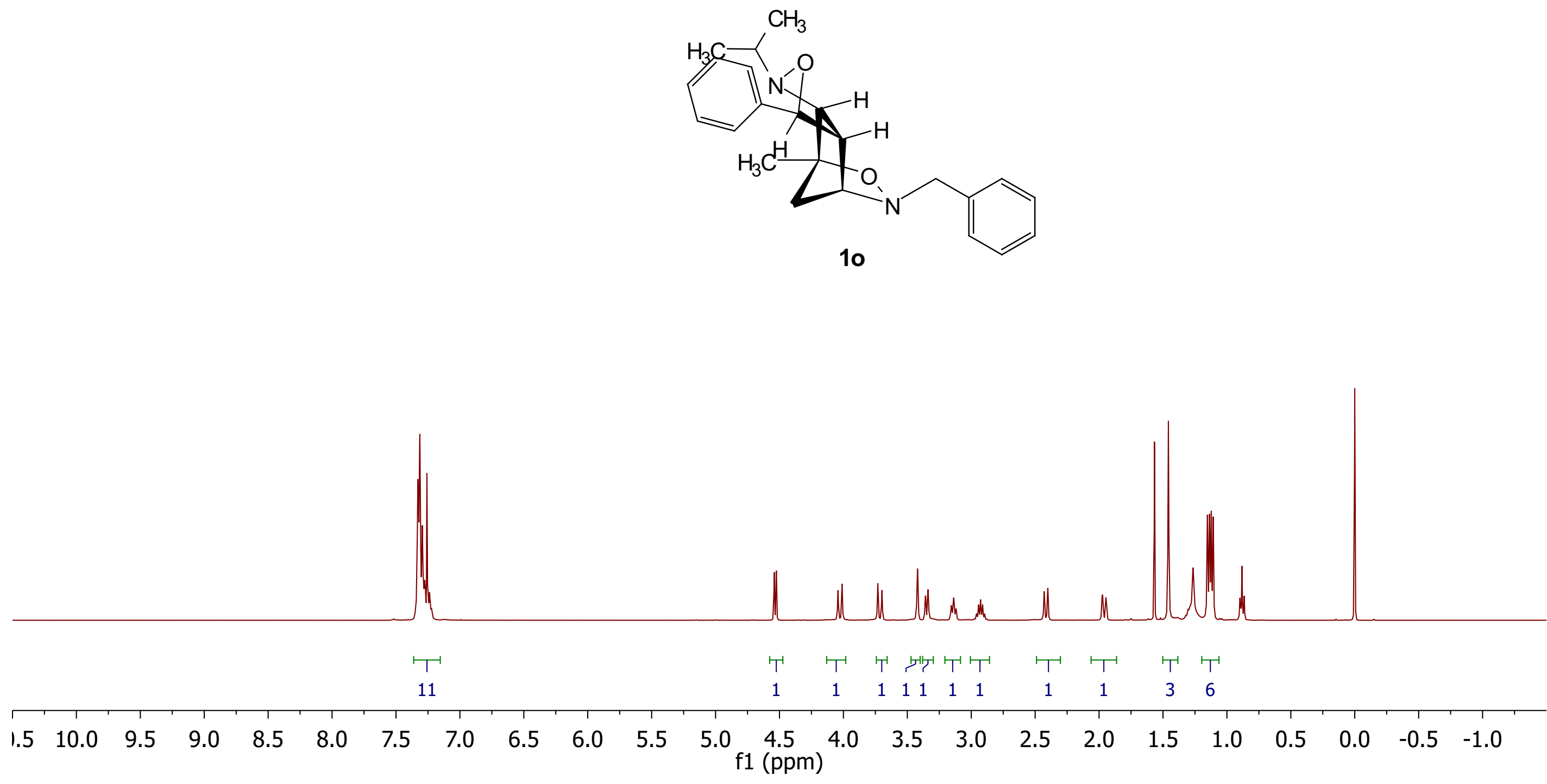
${ }^{13} \mathrm{C}-\mathrm{NMR} 100 \mathrm{MHz}$

in chloroform-d
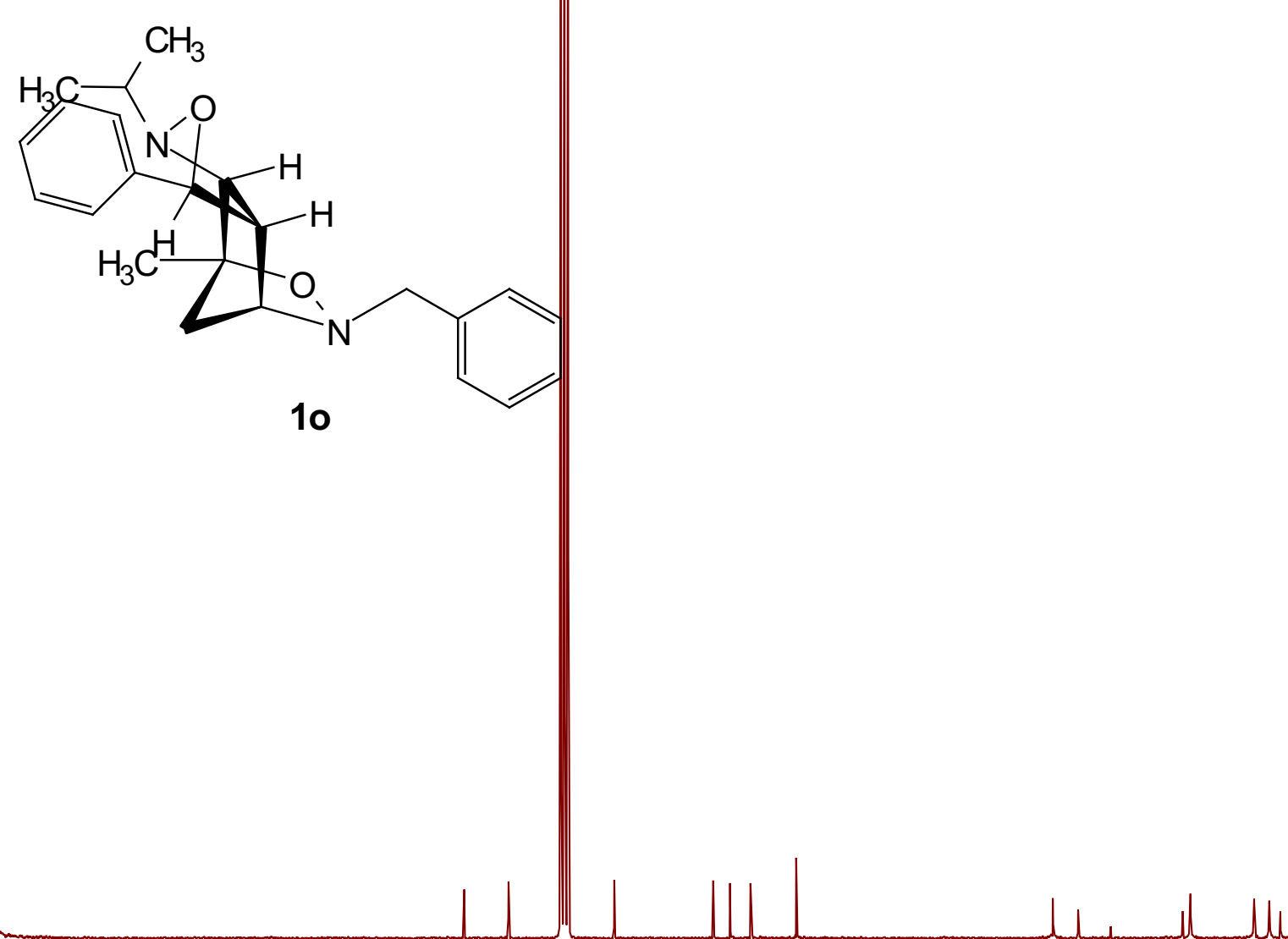

$\begin{array}{llllllllll}1 & 1 & 1 & 1 & 1 & 1 & 1 & 1 & 1 & 1\end{array}$




\section{PD275-3-1-H.1.1.1r}

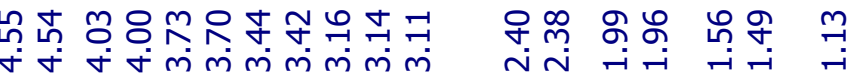
许

\section{${ }^{1} \mathrm{H}-\mathrm{NMR} 400 \mathrm{MHz}$}

in chloroform-d

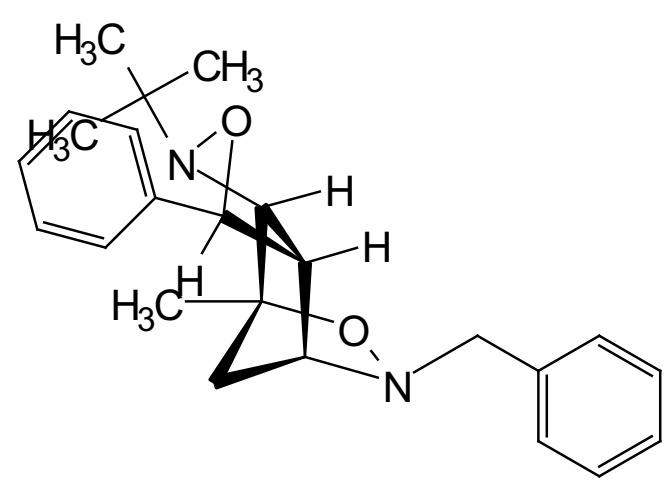

$1 p$

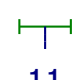

T

$1 \quad \begin{array}{llll}1 & 1 & 2 & 1\end{array}$

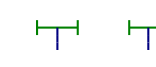

† 
${ }^{13} \mathrm{C}-\mathrm{NMR} 100 \mathrm{MHz}$ in chloroform-d

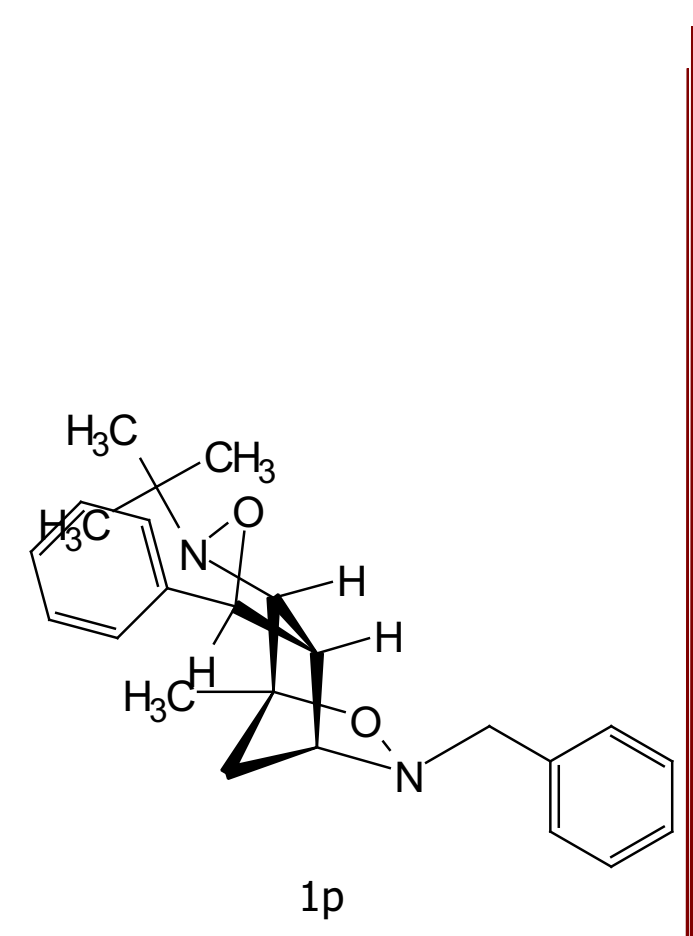

$1 \mathrm{p}$

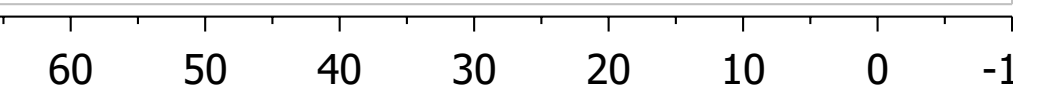


${ }^{1} \mathrm{H}-\mathrm{NMR} 400 \mathrm{MHz}$

in chloroform-d

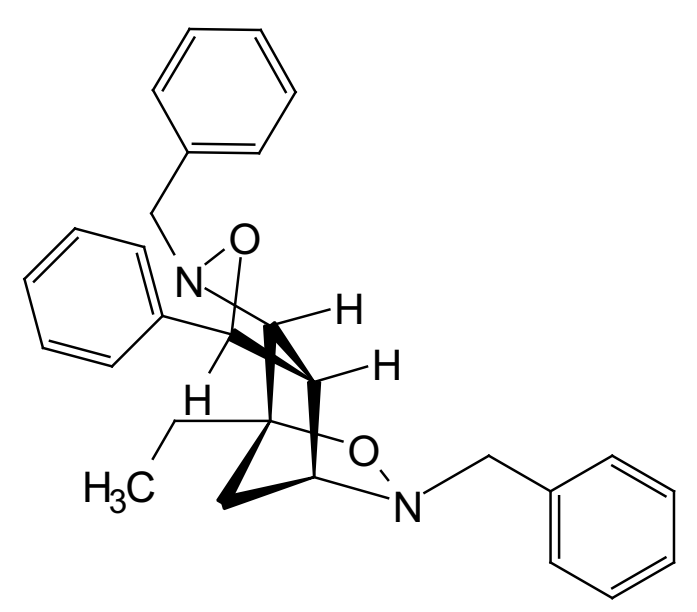

$1 \mathrm{q}$

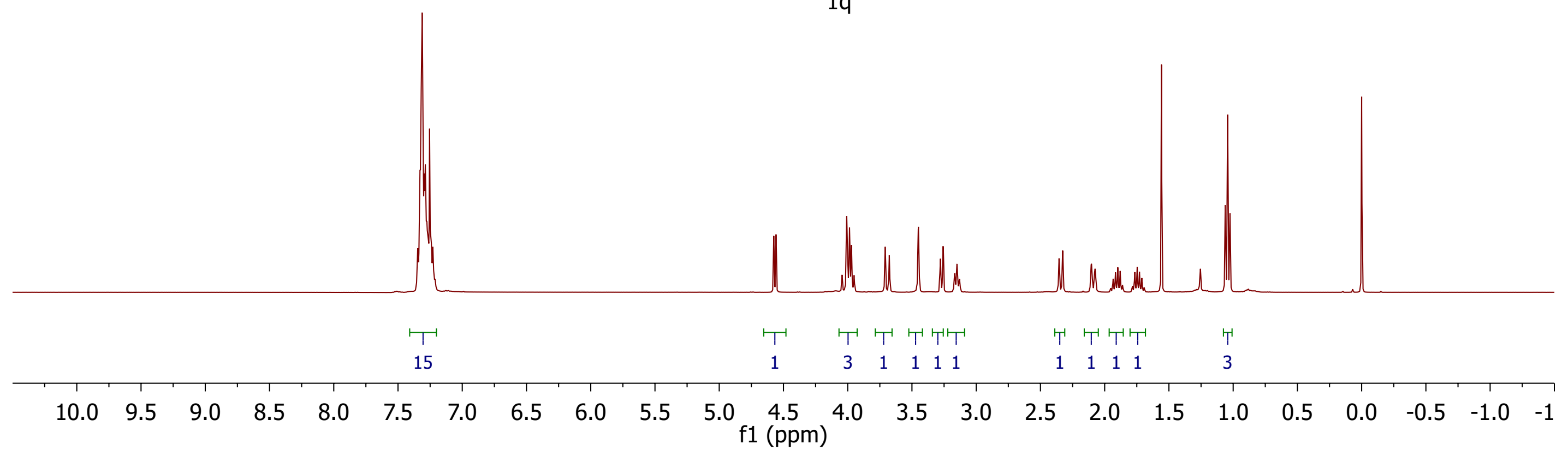




\section{${ }^{13} \mathrm{C}-\mathrm{NMR} 100 \mathrm{MHz}$}

in chloroform-d
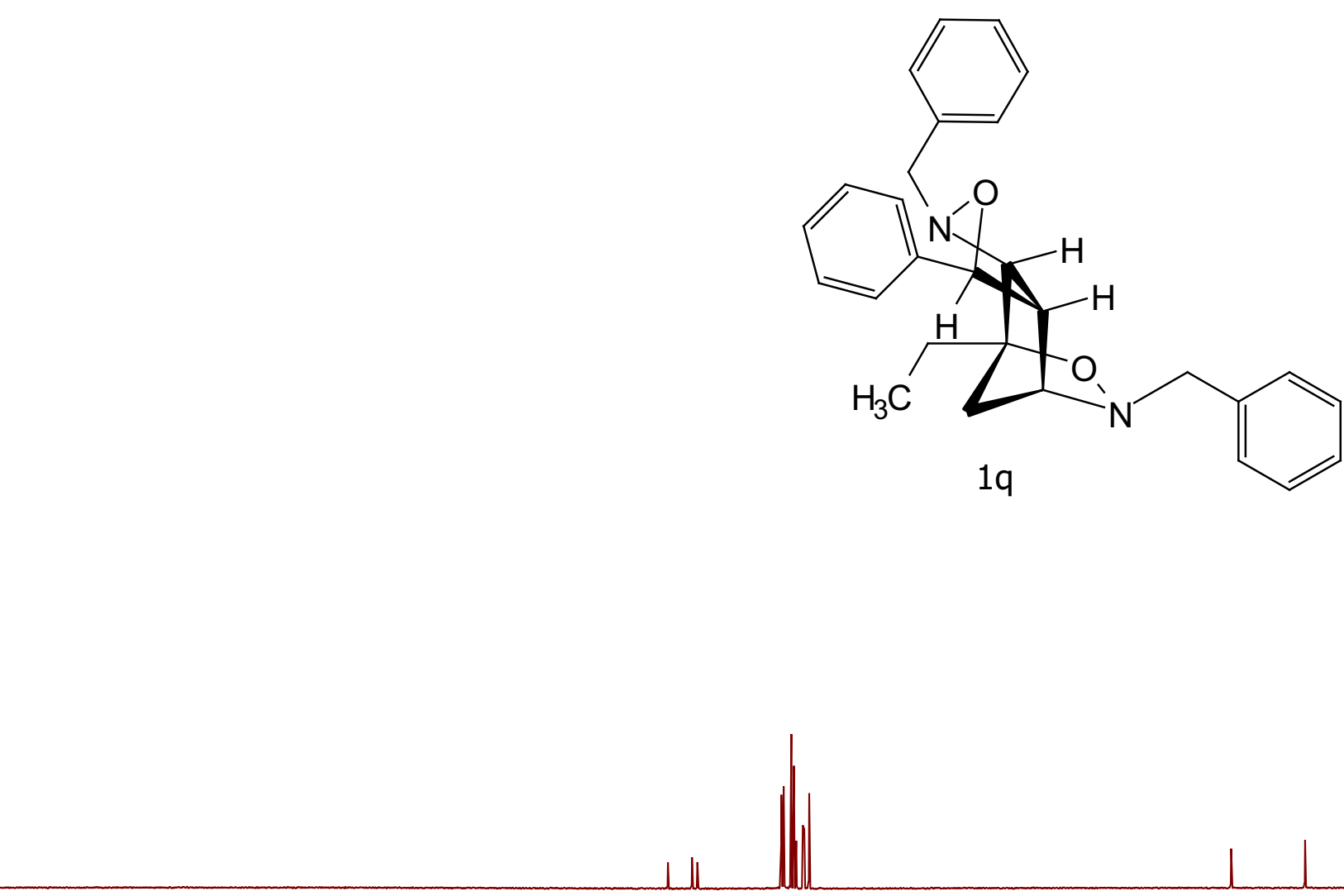

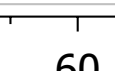

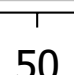

50

50




\section{${ }^{1} \mathrm{H}-\mathrm{NMR} 400 \mathrm{MHz}$} in chloroform-d

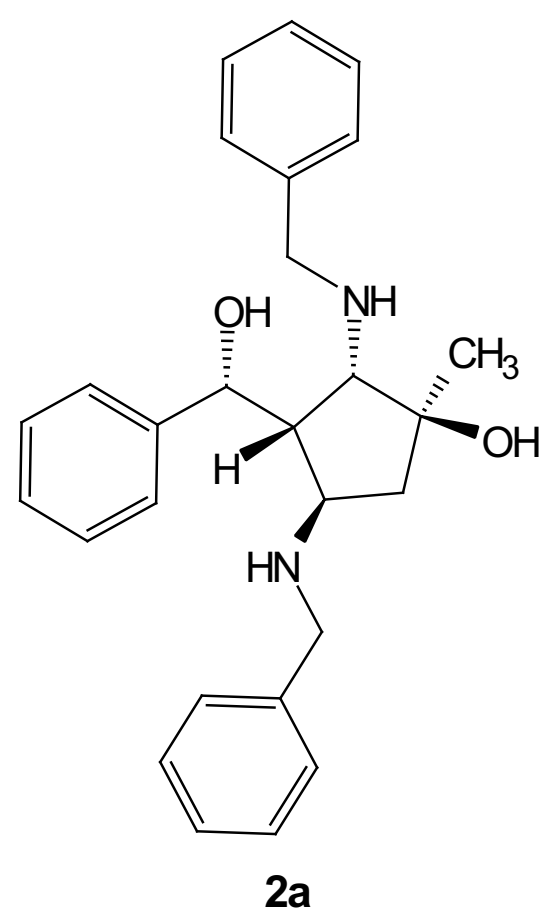

$2 a$

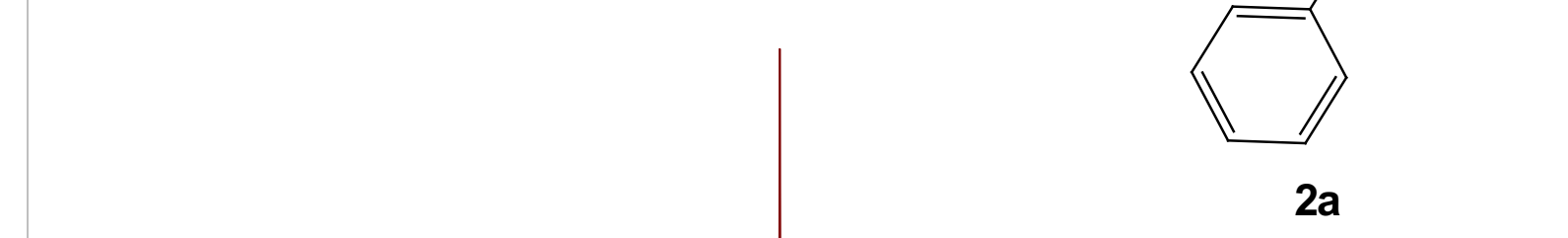

\begin{tabular}{llllllllllllllllllllllllllll}
\hline .5 & 10.0 & 9.5 & 9.0 & 8.5 & 8.0 & 7.5 & 7.0 & 6.5 & 6.0 & 5.5 & 5.0 & $\begin{array}{c}4.5 \\
f 1(\mathrm{ppm})\end{array}$ & 4.0 & 3.5 & 3.0 & 2.5 & 2.0 & 1.5 & 1.0 & 0.5 & 0.0 & -0.5 & -1.0 & -1
\end{tabular}


${ }^{13} \mathrm{C}-\mathrm{NMR} 100 \mathrm{MHz}$ in chloroform-d
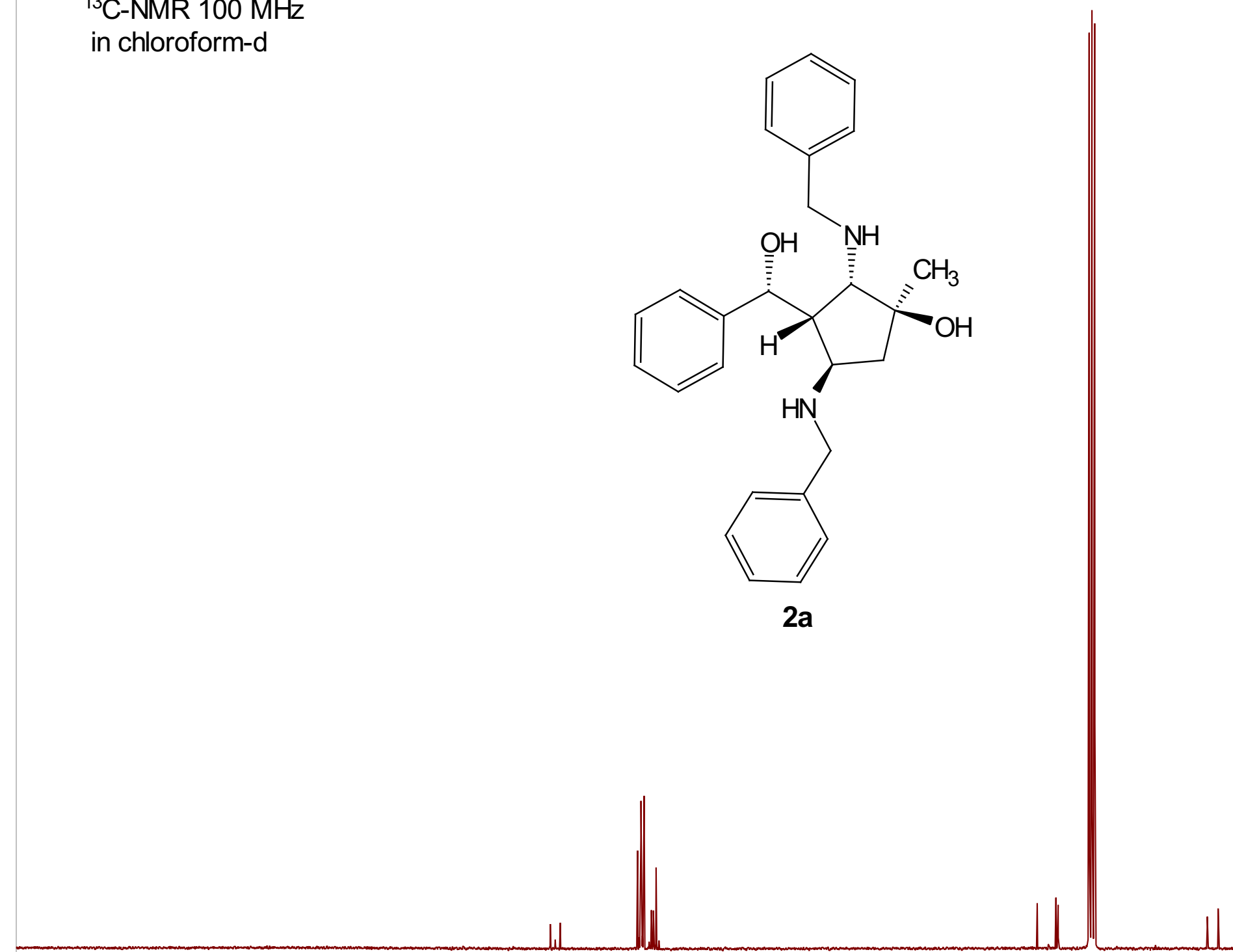


\section{${ }^{1} \mathrm{H}-\mathrm{NMR} 400 \mathrm{MHz}$ \\ in chloroform-d}
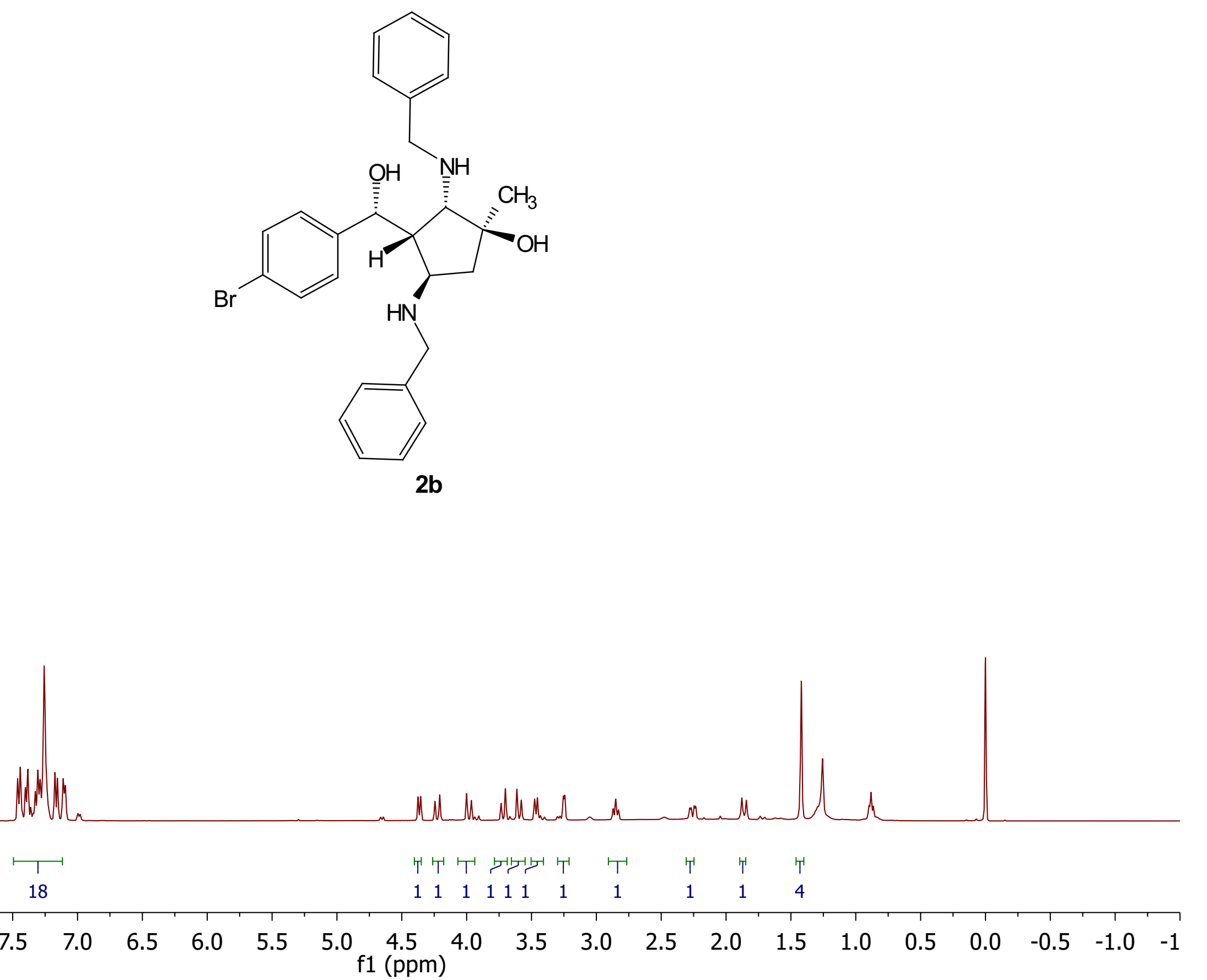
${ }^{13} \mathrm{C}-\mathrm{NMR} 100 \mathrm{MHz}$ in chloroform-d

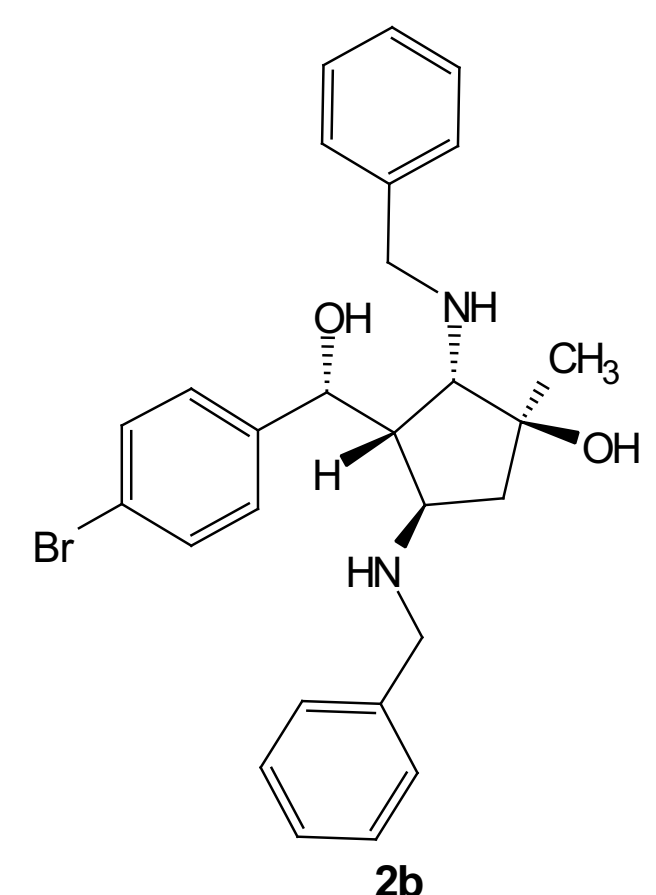

$2 b$ 


\section{${ }^{1} \mathrm{H}-\mathrm{NMR} 400 \mathrm{MHz}$}

in chloroform-d
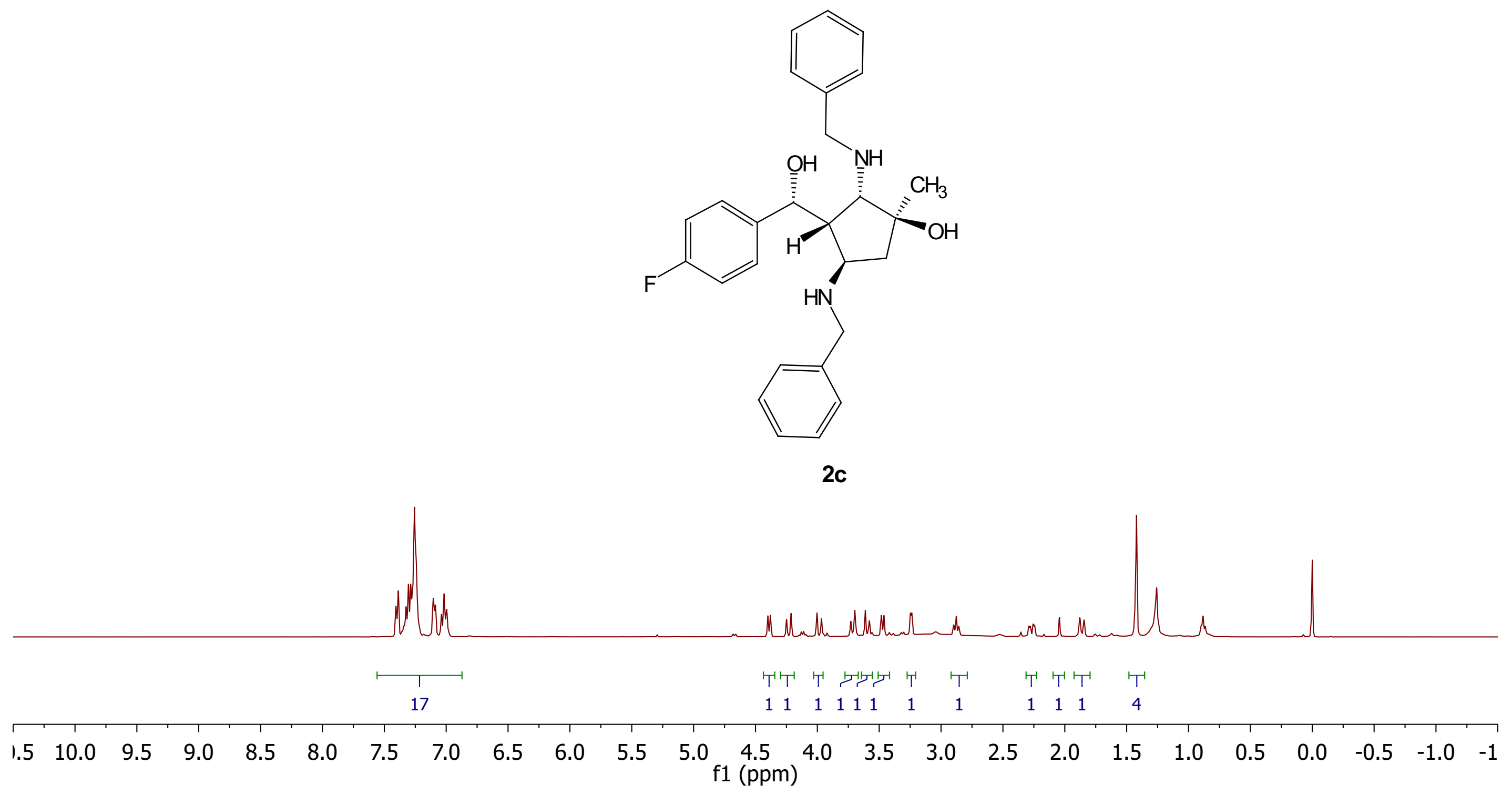


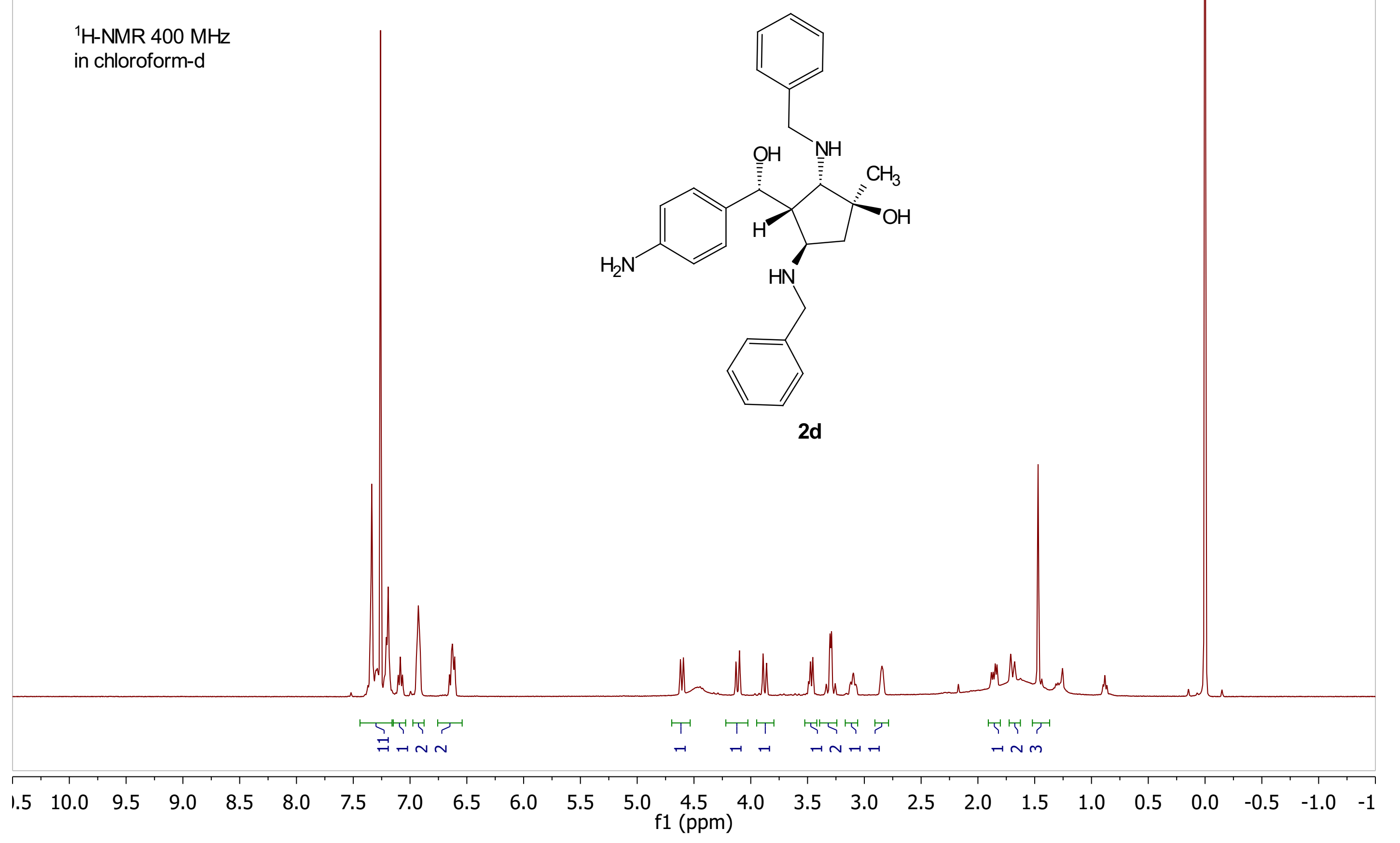




\section{PD181-C-1.10.1.1r}

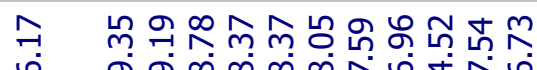

守

$\rightarrow \quad$

${ }^{13} \mathrm{C}-\mathrm{NMR} 100 \mathrm{MHz}$

in chloroform-d

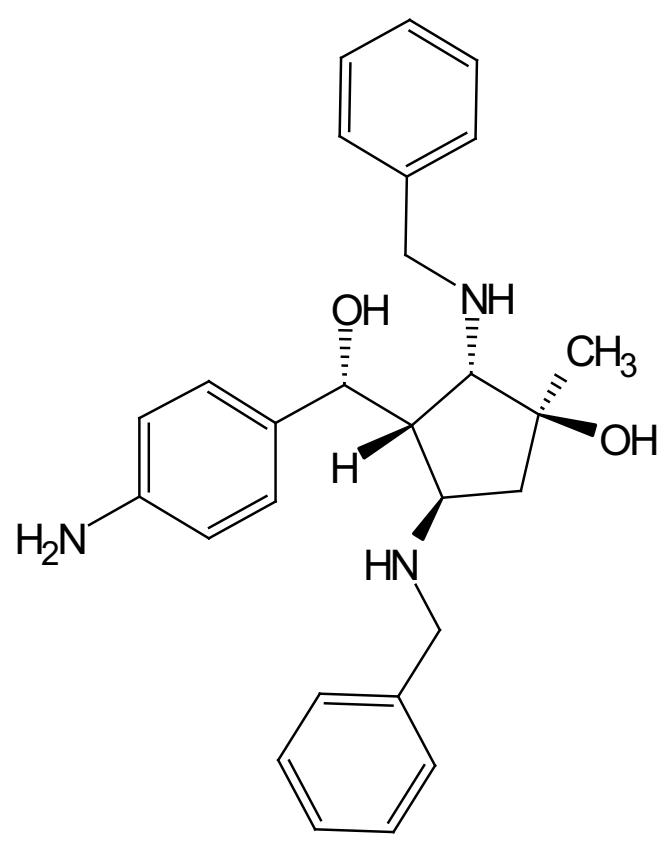

2d

$190 \quad 180$

170

160

$150 \quad 140$

130

120

110

10090

f1 (ppm) 
${ }^{1} \mathrm{H}-\mathrm{NMR} 400 \mathrm{MHz}$ in chloroform-d

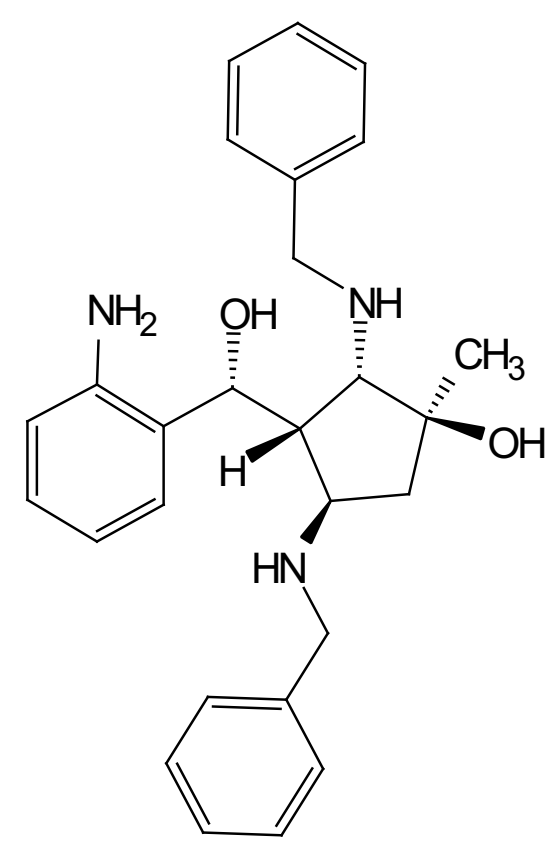

$2 e$

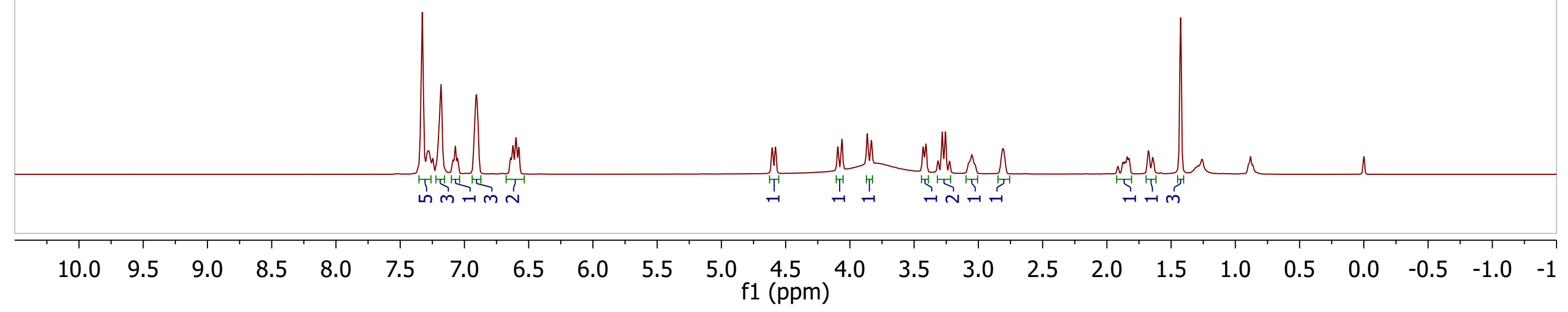


${ }^{13} \mathrm{C}-\mathrm{NMR} 100 \mathrm{MHz}$ in chloroform-d

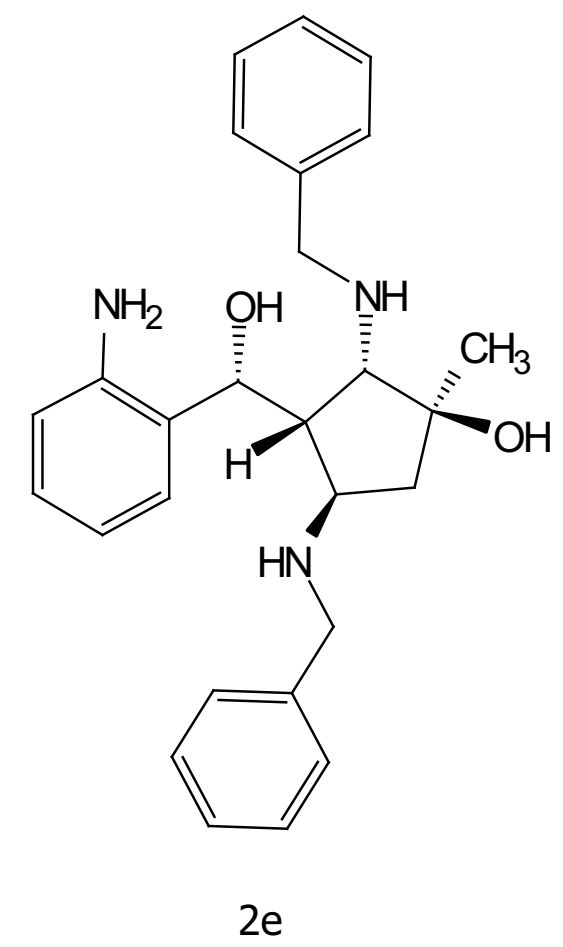

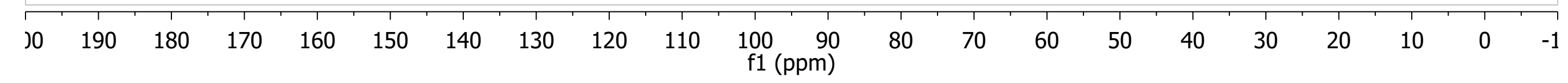


${ }^{1} \mathrm{H}-\mathrm{NMR} 400 \mathrm{MHz}$

in chloroform-d
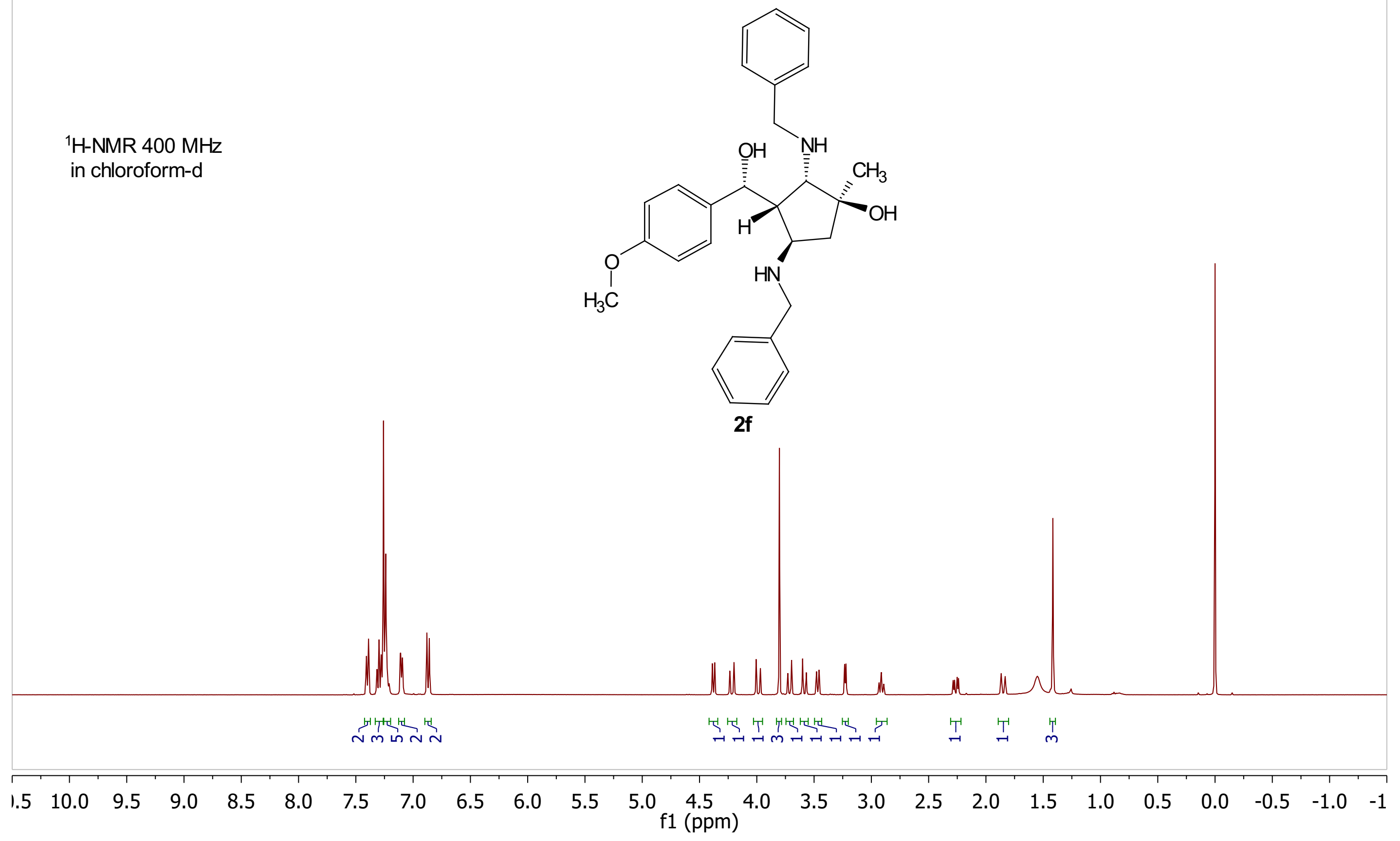
${ }^{13} \mathrm{C}-\mathrm{NMR} 100 \mathrm{MHz}$ in chloroform-d
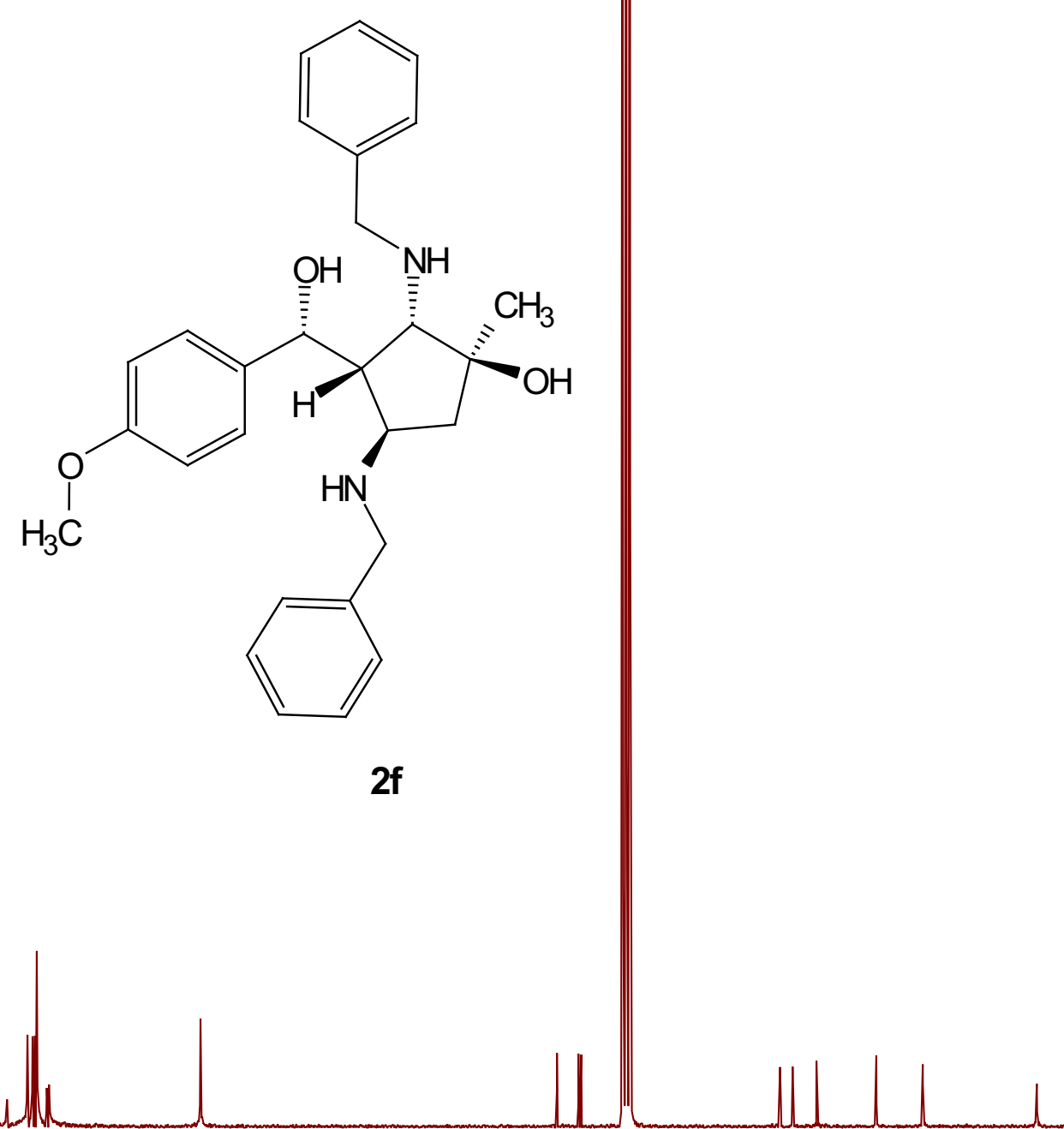

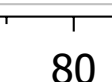

80

70

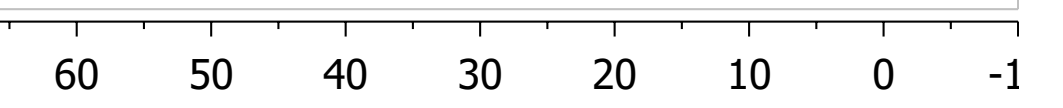




\section{${ }^{1} \mathrm{H}-\mathrm{NMR} 400 \mathrm{MHz}$}

in chloroform-d

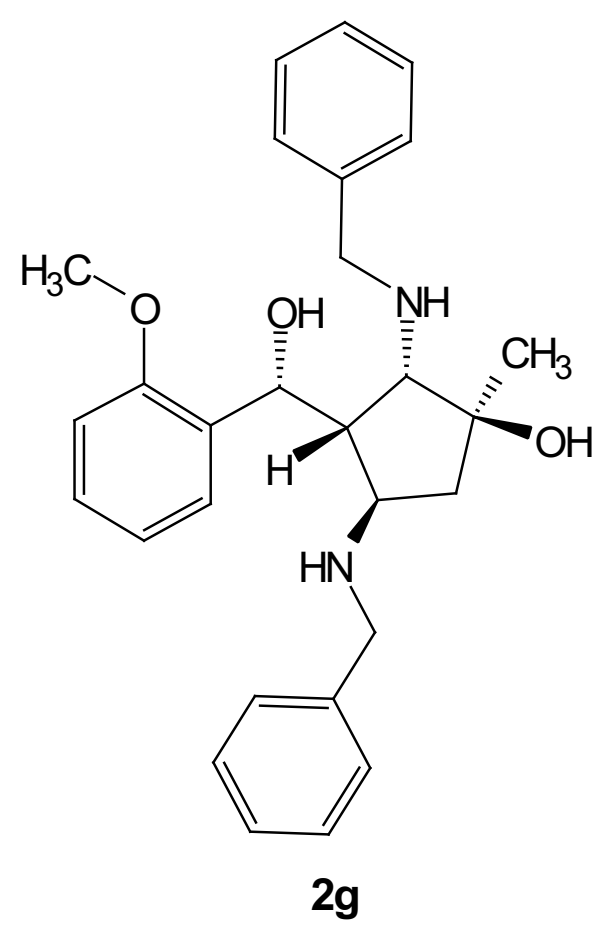




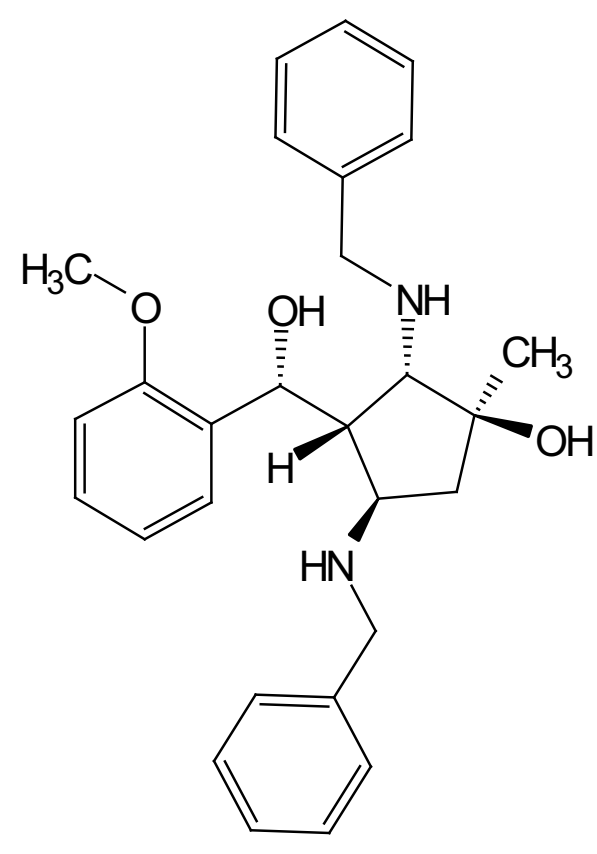

$2 g$ 


\section{${ }^{1} \mathrm{H}-\mathrm{NMR} 400 \mathrm{MHz}$}

in chloroform-d
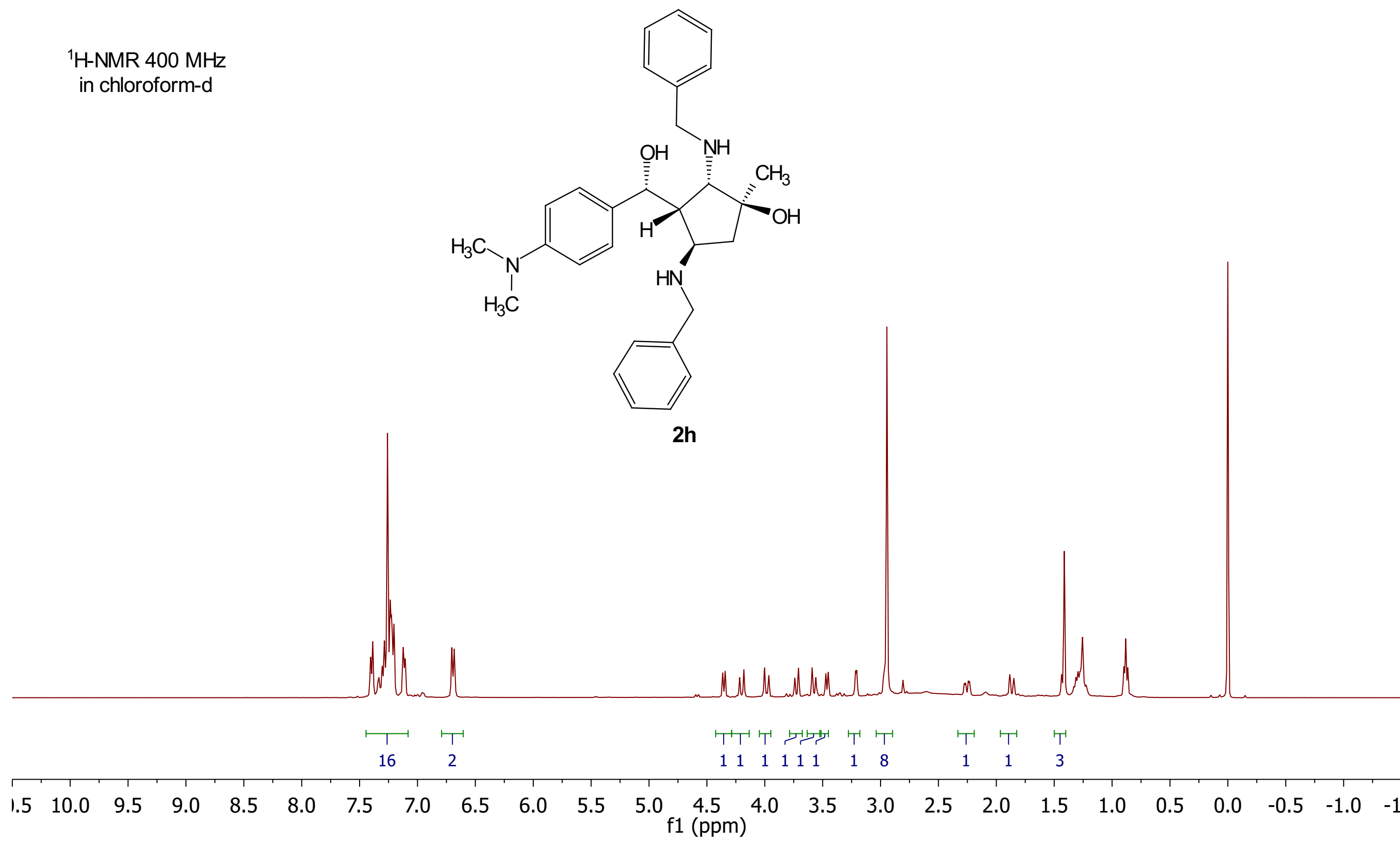
${ }^{13} \mathrm{C}-\mathrm{NMR} 100 \mathrm{MHz}$ in chloroform-d
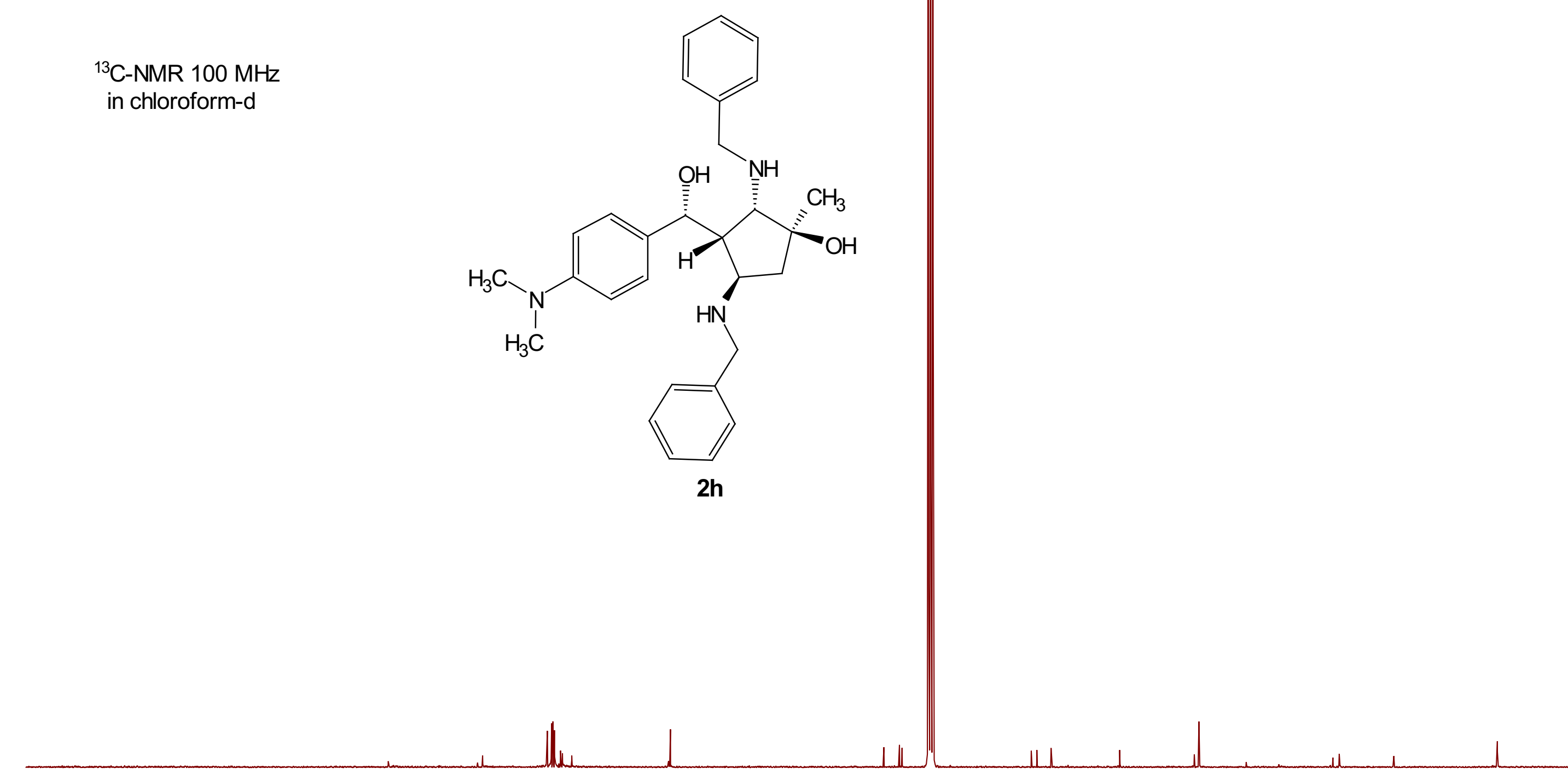

政

180

170

160

150

140

130

120

110

10090

f1 (ppm)

$80 \quad 70$

60

50

40

30

20

$10 \quad 001$ 
H-NMR $400 \mathrm{MHz}$ in chloroform-d
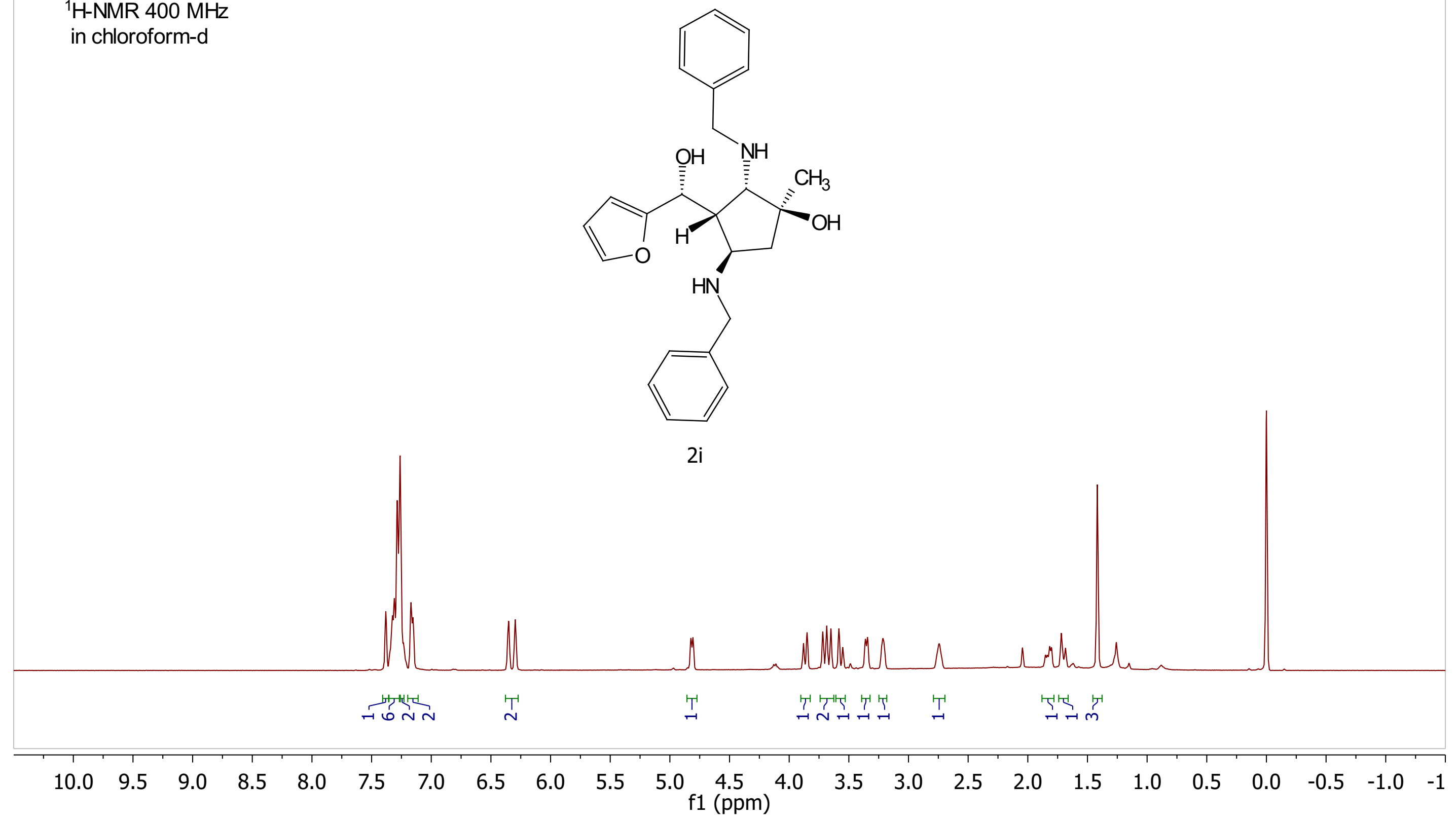
${ }^{13} \mathrm{C}-\mathrm{NMR} 100 \mathrm{MHz}$ in chloroform-d

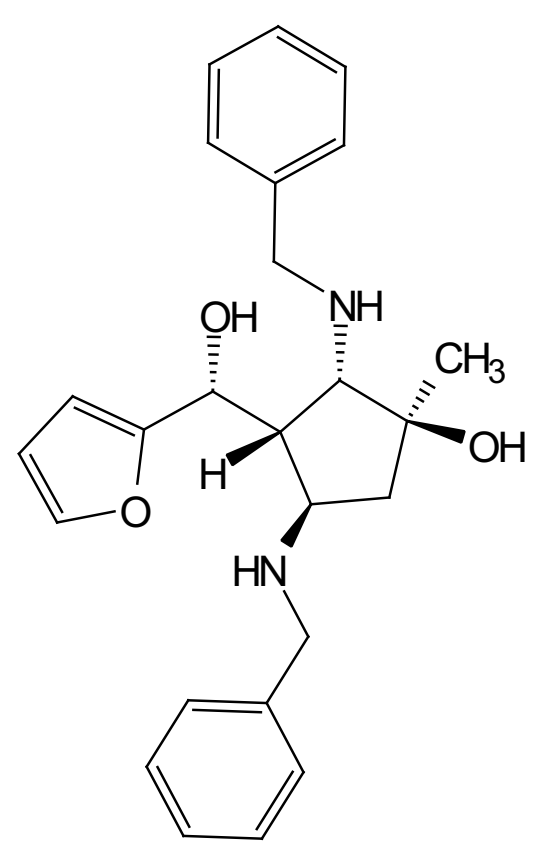




\section{${ }^{1} \mathrm{H}-\mathrm{NMR} 400 \mathrm{MHz}$}

in chloroform-d
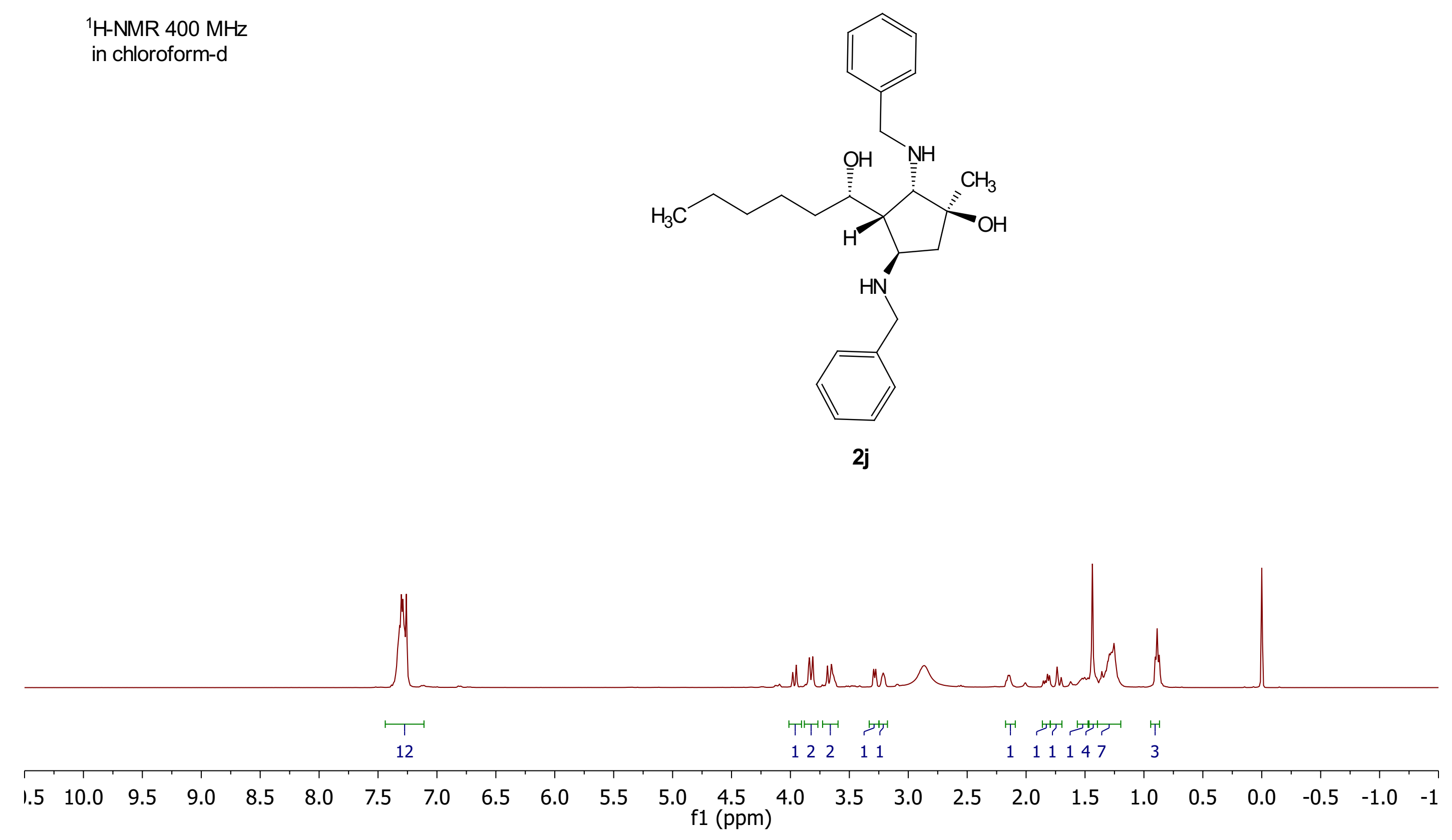


\section{PD252-C.10.1.1r}

${ }^{13} \mathrm{C}-\mathrm{NMR} 100 \mathrm{MHz}$

\section{in chloroform-d}

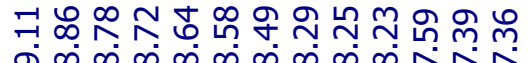

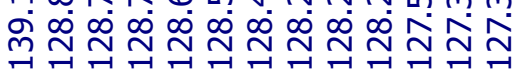

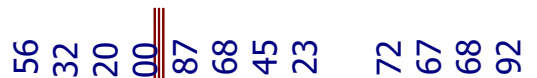

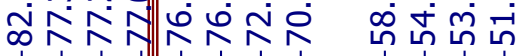


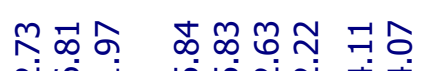
รั

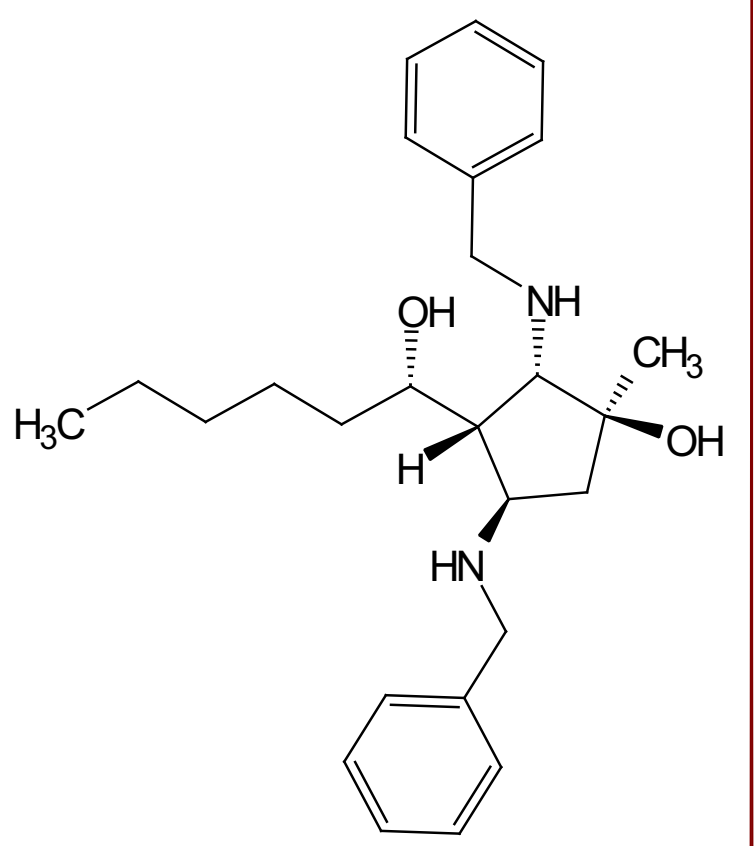

2j 
${ }^{1} \mathrm{H}-\mathrm{NMR} 400 \mathrm{MHz}$ in chloroform-d
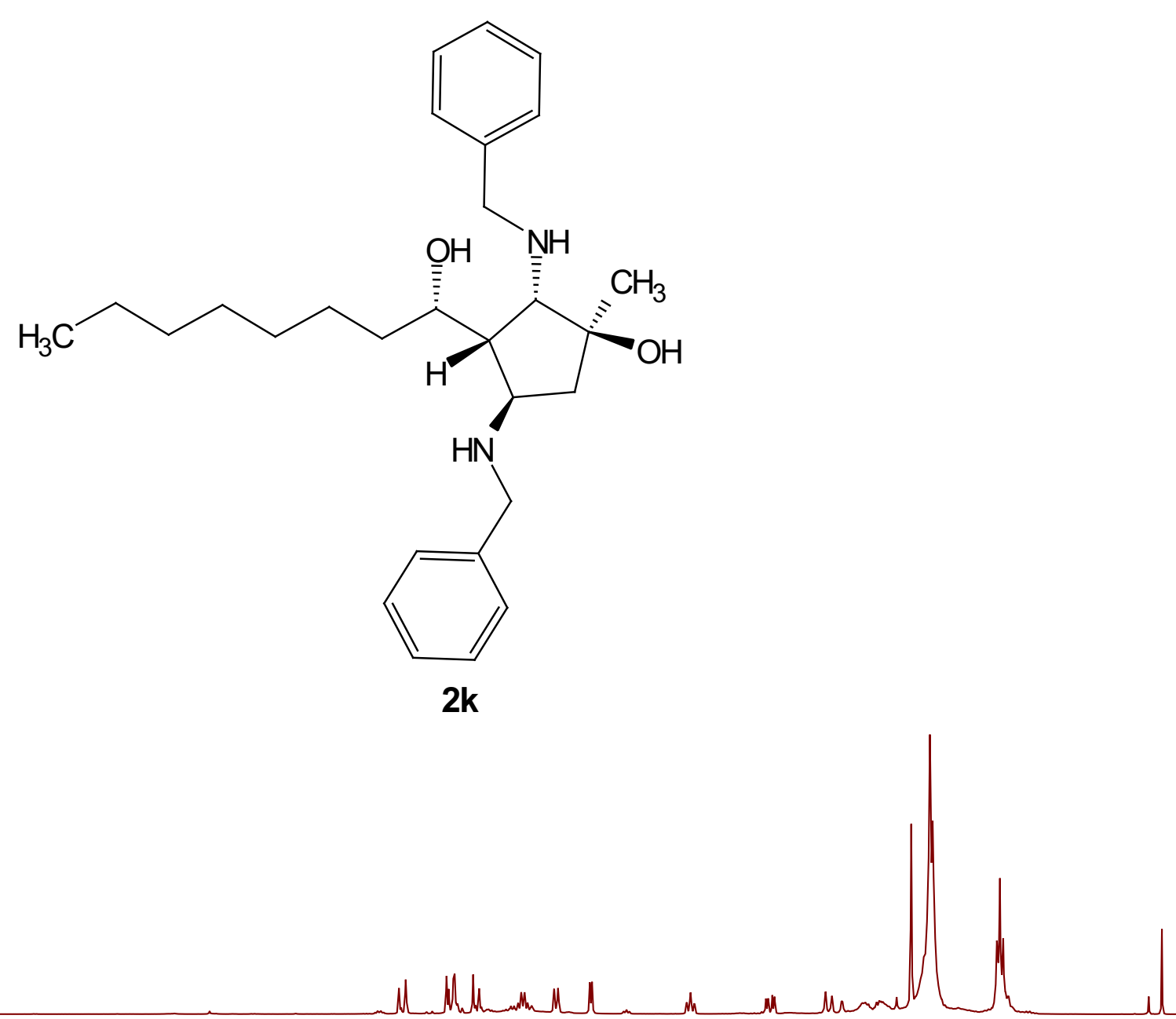

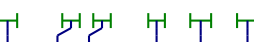

$\begin{array}{llllllllllllllll}1 & 2 & 1 & 1 & 1 & 1 & 1 & 1 & 1 & 1 & 1 & 2 & 3 & 20 & 6\end{array}$

†

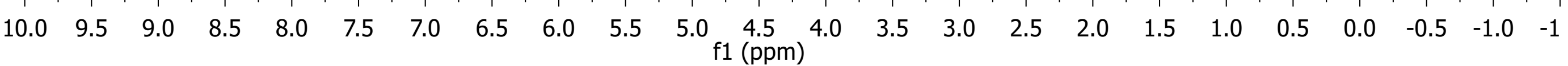


${ }^{13} \mathrm{C}-\mathrm{NMR} 100 \mathrm{MHz}$

in chloroform-d
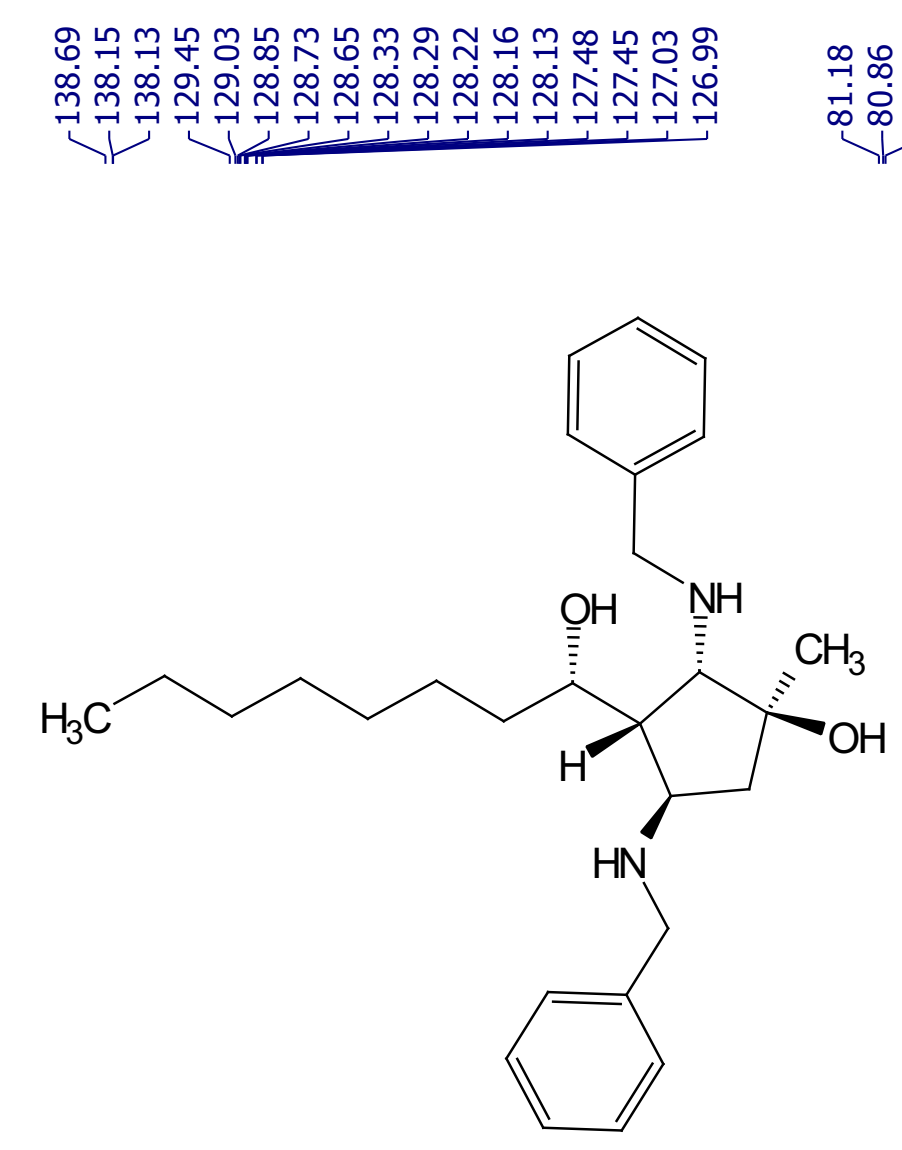

2k 
${ }^{1} \mathrm{H}-\mathrm{NMR} 400 \mathrm{MHz}$ in chloroform-d
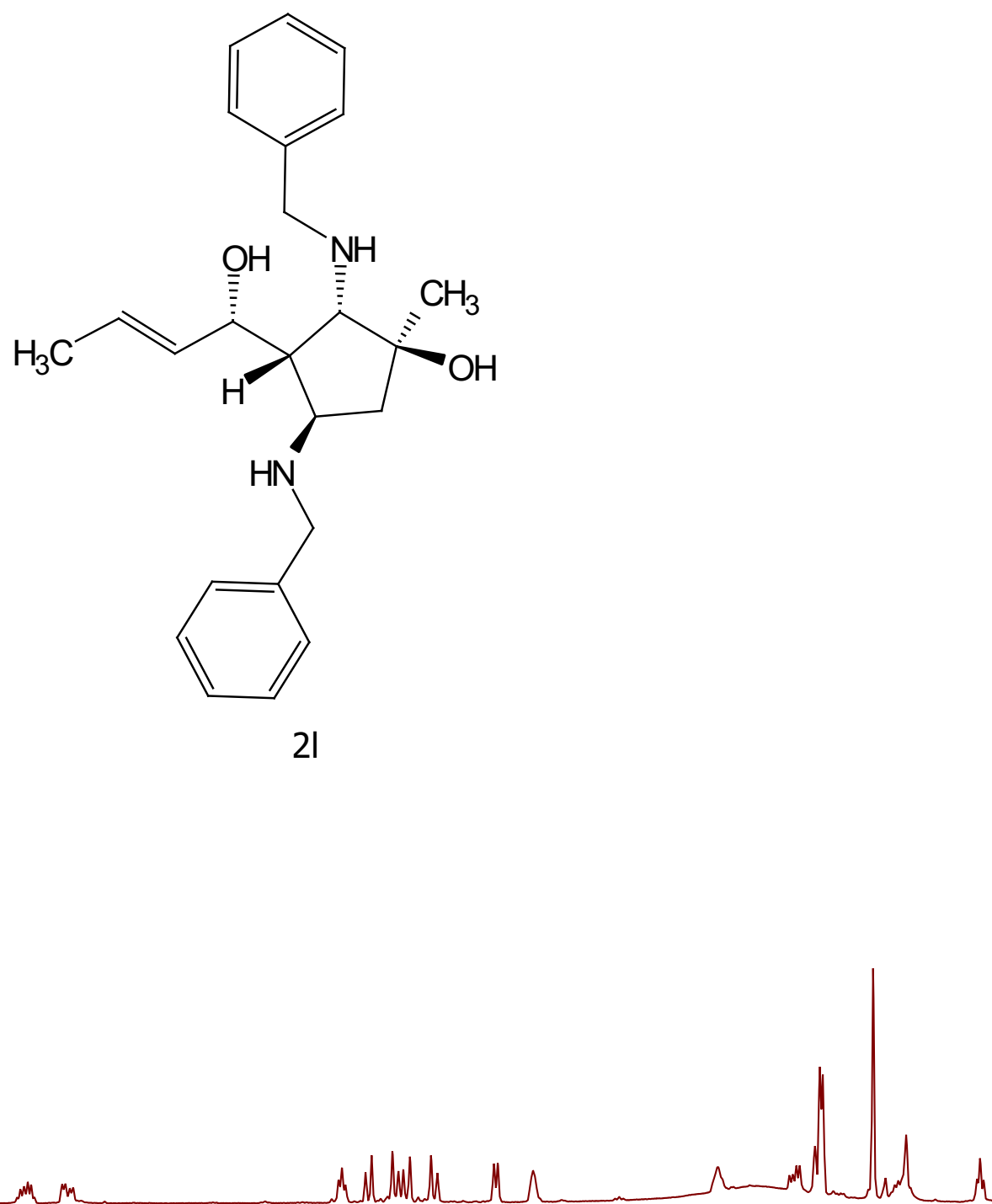

\begin{tabular}{|c|c|c|c|c|c|c|c|c|c|c|c|c|c|c|c|c|c|c|c|c|c|c|c|}
\hline & & & & & & † & & & & $-1 T^{\prime}$ & & & 'T'T' T' & & $\top^{\top}$ & & ? & $T$ & & & & & \\
\hline & & & & & & 12 & & & & 1 & & & 112 & 1 & 1 & & 2 & 3 & & & & & \\
\hline$) .5$ & 10.0 & 9.5 & 9.0 & 8.5 & 8.0 & 7.5 & 7.0 & 6.5 & 6.0 & 5.5 & 5.0 & $\begin{array}{l}4.5 \\
\text { (ppm }\end{array}$ & )$^{4.0}$ & 3.5 & 3.0 & 2.5 & 2.0 & 1.5 & 1.0 & 0.5 & 0.0 & -0.5 & -1.0 \\
\hline
\end{tabular}


${ }^{13} \mathrm{C}-\mathrm{NMR} 100 \mathrm{MHz}$ in chloroform-d

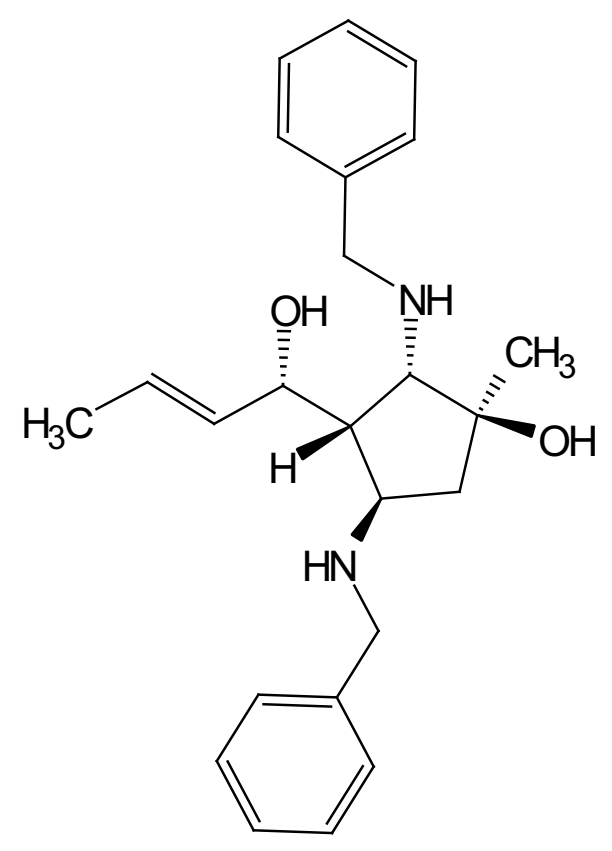

2I

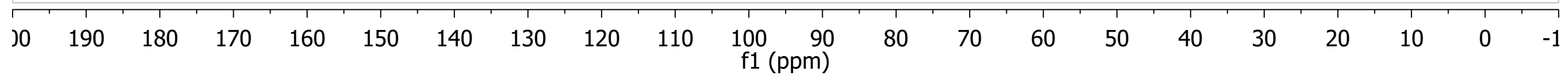




\section{H-NMR $400 \mathrm{MHz}$}

in chloroform-d

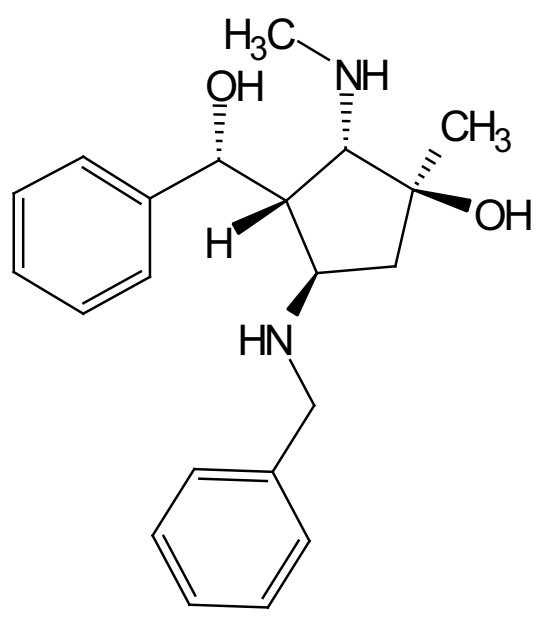

$2 m$

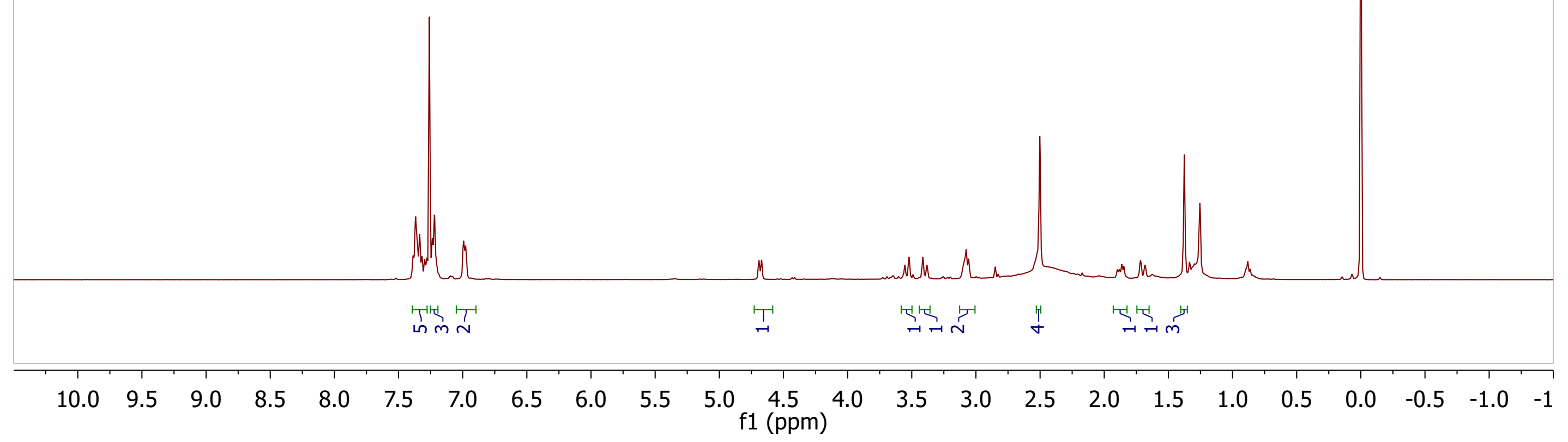


${ }^{13} \mathrm{C}-\mathrm{NMR} 100 \mathrm{MHz}$

in chloroform-d

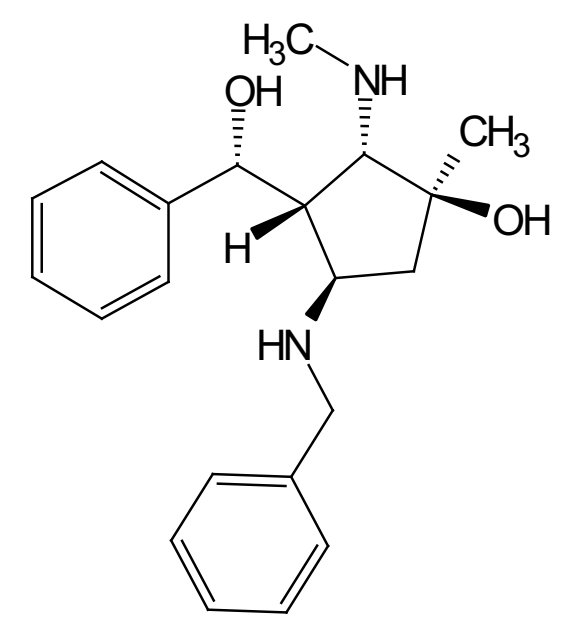

$2 \mathrm{~m}$

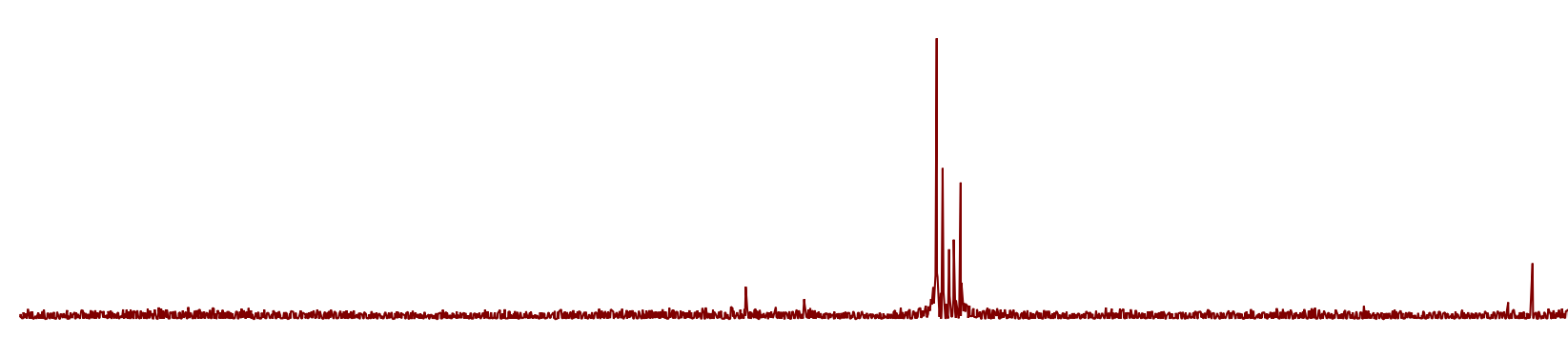




\section{${ }^{13} \mathrm{C}-\mathrm{NMR} 100 \mathrm{MHz}$}

in chloroform-d

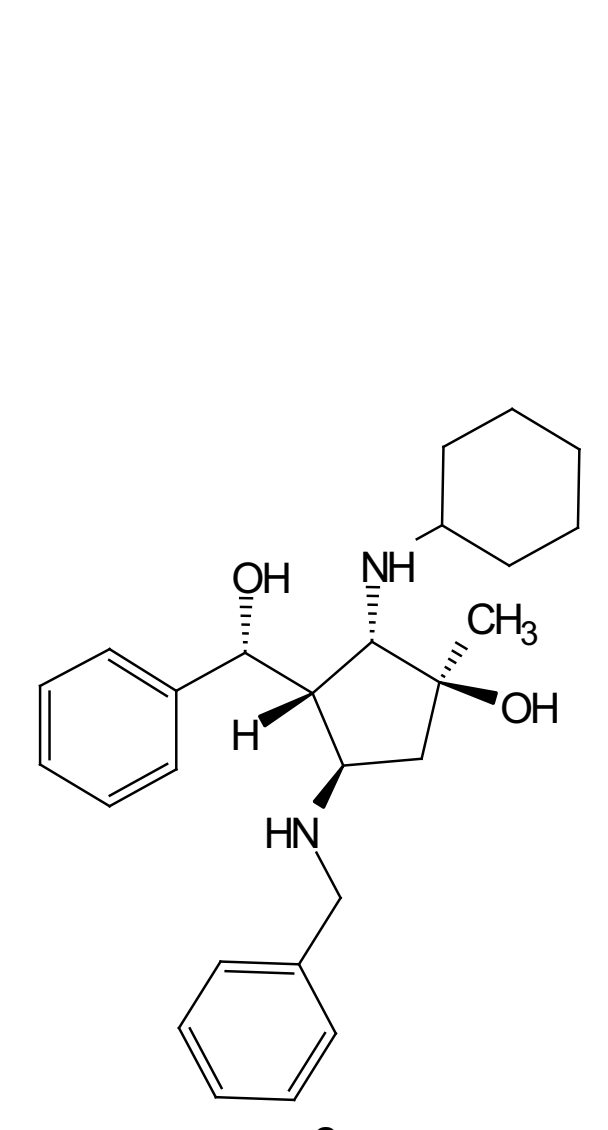

$2 n$ 


\section{${ }^{1} \mathrm{H}-\mathrm{NMR} 400 \mathrm{MHz}$}

in chloroform-d

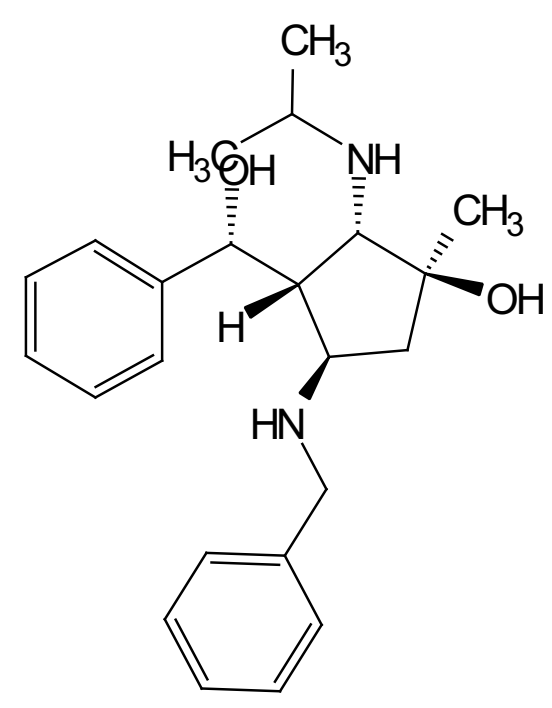

20

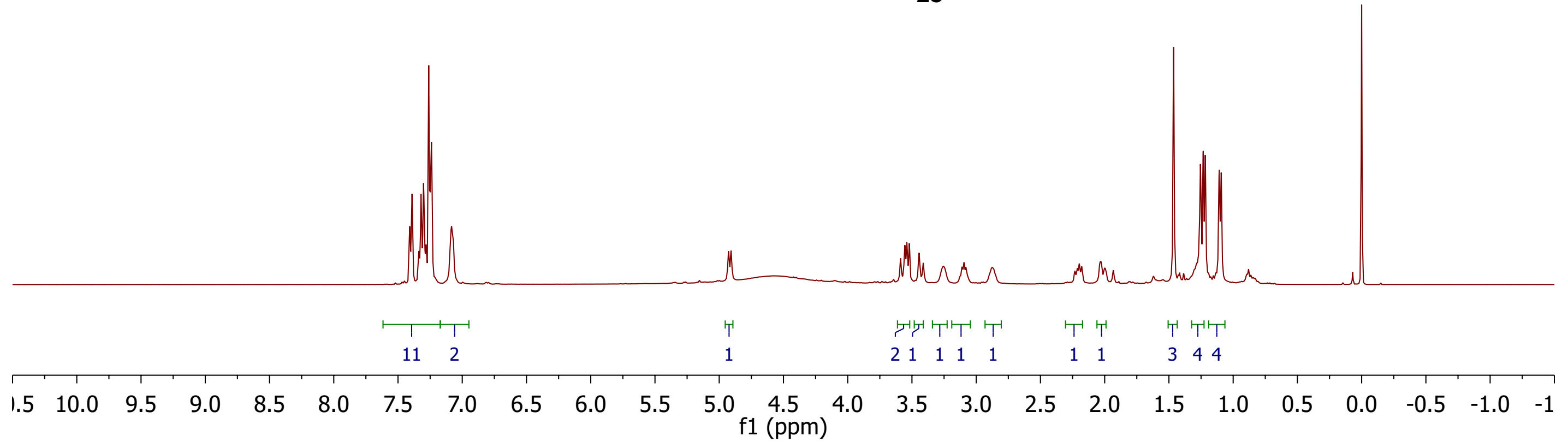


${ }^{13} \mathrm{C}-\mathrm{NMR} 100 \mathrm{MHz}$

in chloroform-d

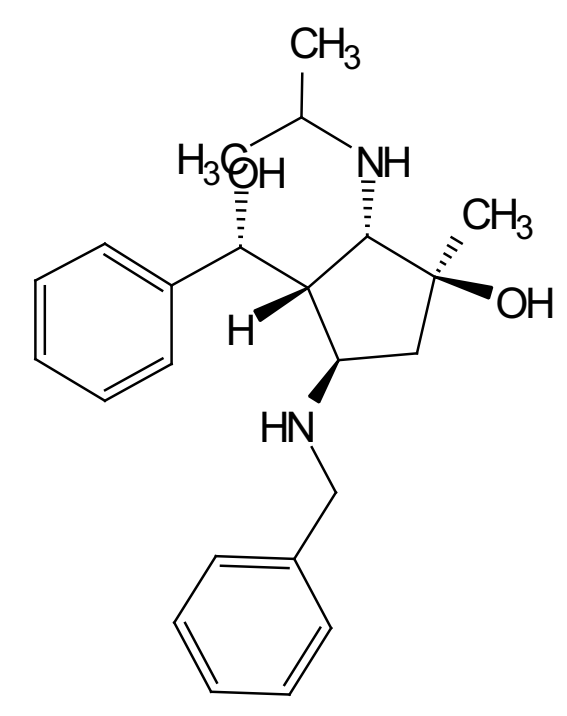

20 


\section{${ }^{1} \mathrm{H}-\mathrm{NMR} 400 \mathrm{MHz}$}

in chloroform-d
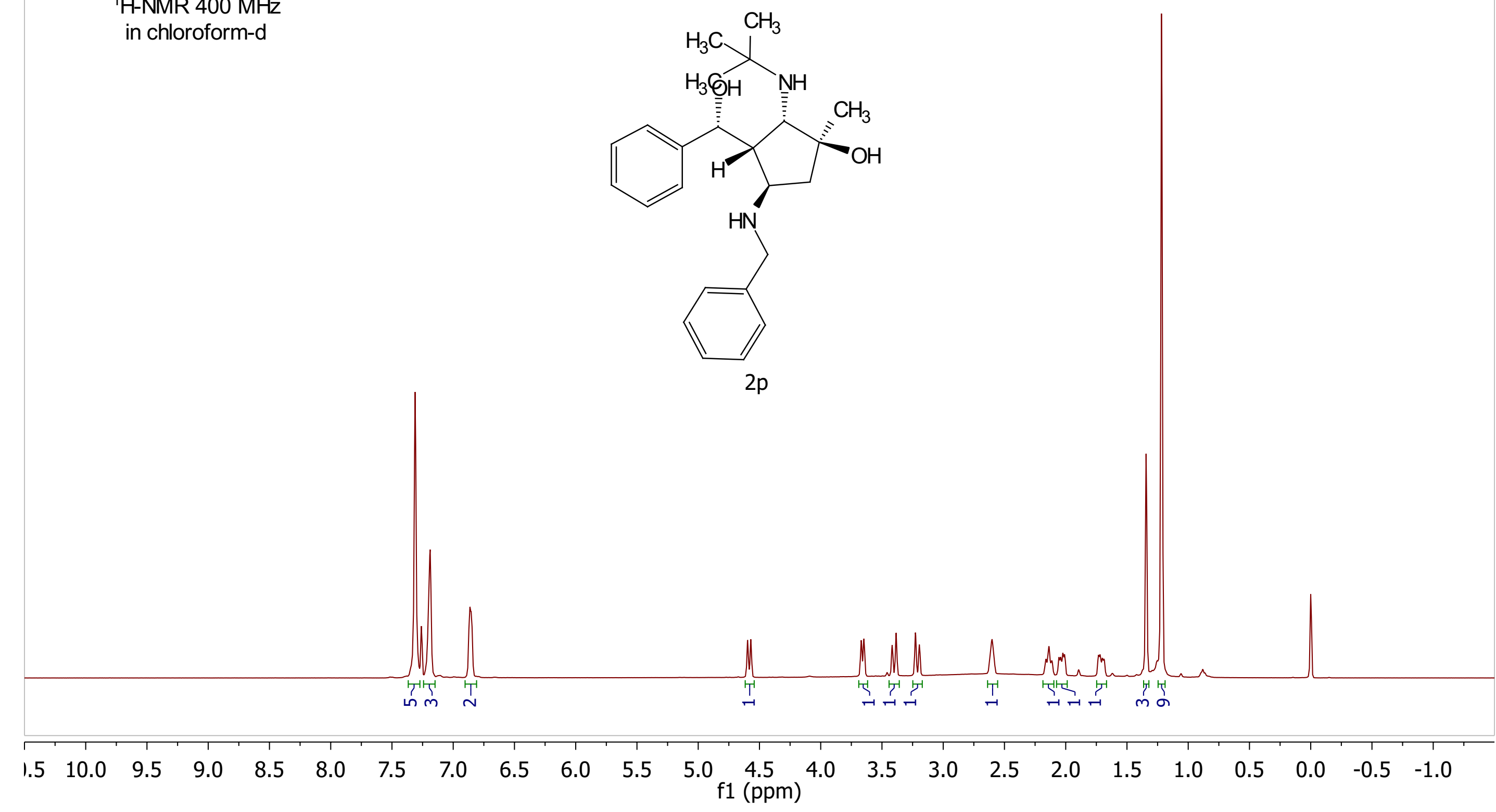
${ }^{13} \mathrm{C}-\mathrm{NMR} 100 \mathrm{MHz}$ in chloroform-d

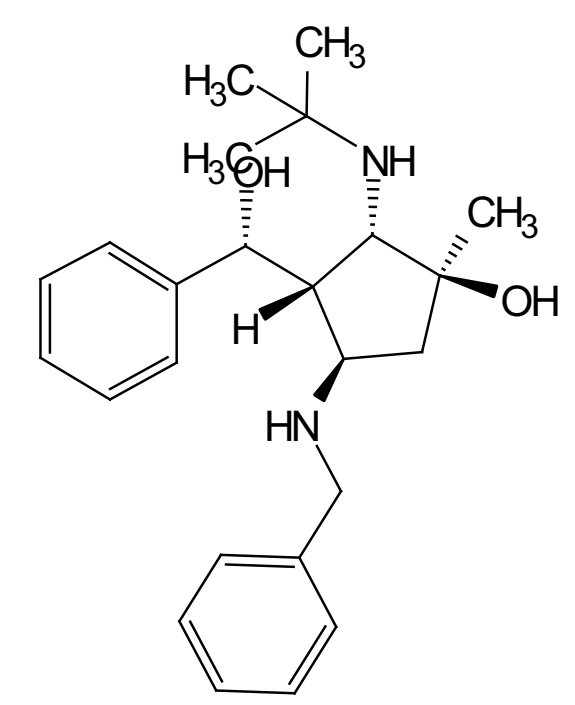

$2 p$
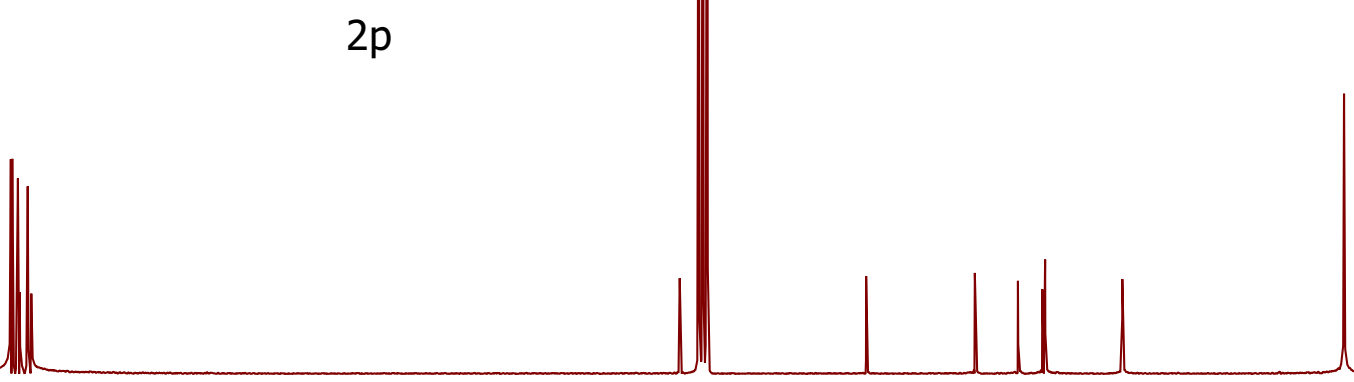

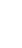

$180 \quad 180$

170

160

$150 \quad 140$

130

120

$\begin{array}{lll}110 & 100 & 90\end{array}$

f1 (ppm)

80

70

60

50

40

30

20

10

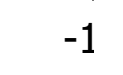




\section{${ }^{1} \mathrm{H}-\mathrm{NMR} 400 \mathrm{MHz}$}

in chloroform-d

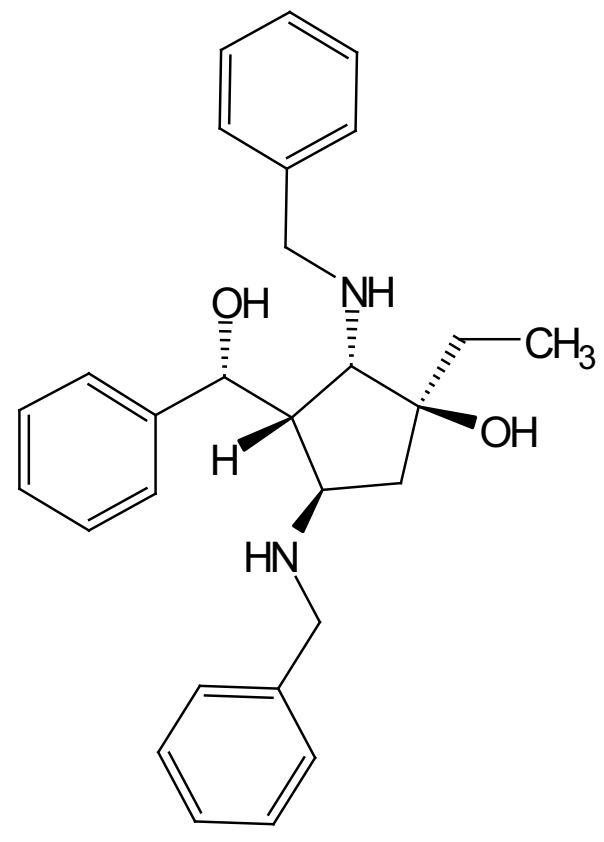

$2 q$

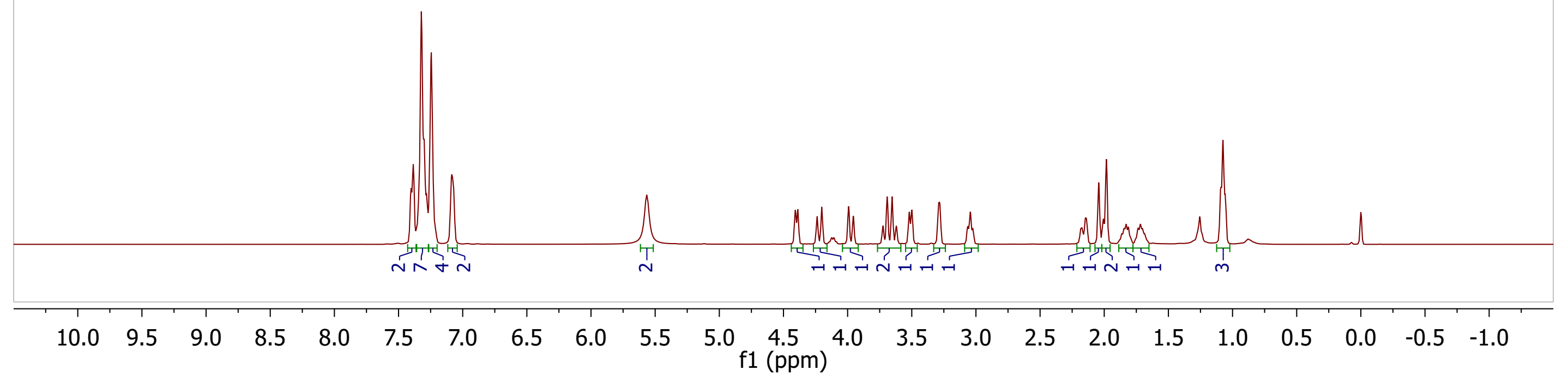




\section{${ }^{13} \mathrm{C}-\mathrm{NMR} 100 \mathrm{MHz}$}

in chloroform-d

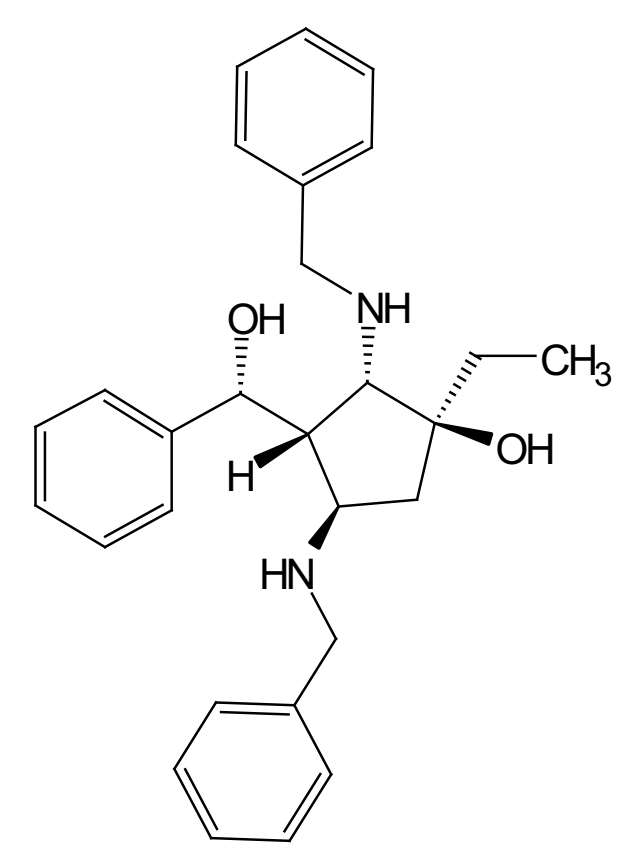

$2 q$

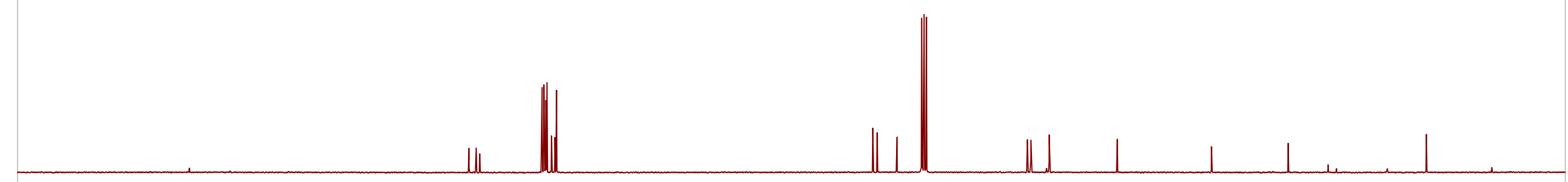

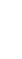

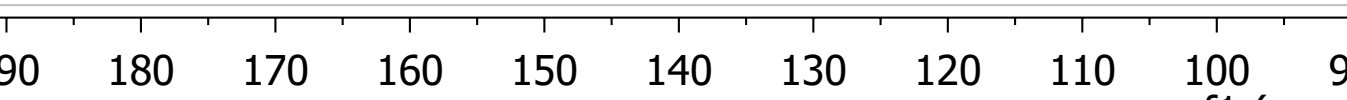
f1 (ppm) 